\title{
Decomposition of Feynman integrals by multivariate intersection numbers
}

\author{
Hjalte Frellesvig, ${ }^{a, b}$ Federico Gasparotto, ${ }^{a, b}$ Stefano Laporta, ${ }^{a, b}$ Manoj K. Mandal, ${ }^{b, a}$ \\ Pierpaolo Mastrolia, ${ }^{a, b}$ Luca Mattiazzi $^{b, a}$ and Sebastian Mizera ${ }^{c}$ \\ ${ }^{a}$ Dipartimento di Fisica e Astronomia, Università di Padova, \\ Via Marzolo 8, 35131 Padova, Italy \\ ${ }^{b}$ INFN, Sezione di Padova, \\ Via Marzolo 8, 35131 Padova, Italy \\ ${ }^{c}$ Institute for Advanced Study, Einstein Drive, \\ Princeton, NJ 08540, U.S.A. \\ E-mail: hjalte.frellesvig@pd.infn.it, federico.gasparotto@pd.infn.it, \\ stefano.laporta@pd.infn.it, manojkumar.mandal@pd.infn.it, \\ pierpaolo.mastrolia@pd.infn.it, luca.mattiazzi@pd.infn.it, \\ smizera@ias.edu
}

Abstract: We present a detailed description of the recent idea for a direct decomposition of Feynman integrals onto a basis of master integrals by projections, as well as a direct derivation of the differential equations satisfied by the master integrals, employing multivariate intersection numbers. We discuss a recursive algorithm for the computation of multivariate intersection numbers, and provide three different approaches for a direct decomposition of Feynman integrals, which we dub the straight decomposition, the bottom-up decomposition, and the top-down decomposition. These algorithms exploit the unitarity structure of Feynman integrals by computing intersection numbers supported on cuts, in various orders, thus showing the synthesis of the intersection-theory concepts with unitarity-based methods and integrand decomposition. We perform explicit computations to exemplify all of these approaches applied to Feynman integrals, paving a way towards potential applications to generic multi-loop integrals.

Keywords: Perturbative QCD, Scattering Amplitudes, Differential and Algebraic Geometry

ArXiv EPrint: 2008.04823 


\section{Contents}

1 Introduction 1

2 Feynman integrals and differential forms $\quad 4$

2.1 The cohomology group and its dual 4

2.2 Dimension of twisted cohomology groups $\quad 6$

$\begin{array}{lll}2.3 & \text { Intersection numbers for twisted (co)homology classes } & 7\end{array}$

2.4 Linear and quadratic relations 8

$\begin{array}{ll}2.5 & \text { Differential equation for forms and dual forms } \\ \end{array}$

3 Multivariate intersection numbers $\quad 11$

$\begin{array}{lll}3.1 & \text { Intersection numbers of logarithmic forms } & 11\end{array}$

$\begin{array}{lll}3.2 & \text { Intersection numbers of general forms } & 12\end{array}$

$\begin{array}{lll}3.3 & \text { Intersection numbers for 1-forms } & 12\end{array}$

$\begin{array}{lll}3.4 & \text { Intersection numbers for 2-forms } & 13\end{array}$

$\begin{array}{ll}3.5 & \text { Intersection numbers for } n \text {-forms } \\ & 16\end{array}$

$\begin{array}{lll}\text { 3.5.1 Explicit formula } & 18\end{array}$

$\begin{array}{ll}3.5 .2 \text { Dual formula } & 19\end{array}$

$\begin{array}{ll}3.6 & \text { Simplifying the computation of intersection numbers } \\ \end{array}$

4 Feynman integral decomposition $\quad 19$

$\begin{array}{ll}4.1 \text { Straight decomposition } & 20\end{array}$

4.2 Bottom-up decomposition 21

4.3 Top-down decomposition 23

5 Examples $\quad \mathbf{2 4}$

5.1 One-loop massless box 24

$\begin{array}{lll}5.1 .1 & \text { Straight decomposition } & 26\end{array}$

$\begin{array}{lll}5.1 .2 & \text { Bottom-up decomposition } & 27\end{array}$

$\begin{array}{lll}5.1 .3 & \text { Top-down decomposition } & 28\end{array}$

$\begin{array}{lll}5.2 & \text { One-loop QED triangle } & 30\end{array}$

$\begin{array}{lll}\text { 5.2.1 Straight decomposition } & 31\end{array}$

5.3 Two-loop QED sunrise $\quad 32$

5.3.1 Bottom-up decomposition 33

5.4 Further examples 34

$\begin{array}{llr}6 & \text { Conclusions } & 39\end{array}$

$\begin{array}{ll}\text { A Master decomposition formula } & 40\end{array}$

B Derivation of the connection $\Omega$ for $n$-form intersection numbers 42 
C Intersection numbers for the three examples

C.1 One-loop massless box 44

C.1.1 Straight decomposition 44

C.1.2 Bottom-up decomposition $\quad 45$

C.1.3 Top-down decomposition $\quad 45$

C.2 One-loop QED triangle 45

C.3 Two-loop QED sunrise 46

\section{Introduction}

Feynman integrals in dimensional regularization admit parametric integral representations which expose their nature as Aomoto-Gel'fand integrals, thereby enabling a novel form of investigation of their algebraic structure by means of intersection theory of twisted de Rham (co)homology for general hypergeometric functions [1-3]. Accordingly, intersection numbers of differential forms [4] can be employed to define a scalar product on a vector space of Feynman integrals [1], such that projecting any multi-loop integral onto a basis of master integrals (MIs) becomes conceptually identical to decomposing a generic vector into a basis of a vector space.

Univariate intersection numbers, as shown in the original studies [1, 2], were sufficient to validate a novel method based on intersection theory for deriving integral relations, which was used for the direct derivation of contiguity relations for Lauricella $F_{D}$ functions, as well as for Feynman integrals on maximal cuts, i.e. with on-shell internal lines, that admit a one-fold integral representations. As proposed in [2], applications of this novel method to the decomposition of full Feynman integrals in terms of a complete set of MIs, including the ones corresponding to subdiagrams, as well as deriving contiguity relations for special functions admitting multi-fold integral representation, required the use of multivariate intersection numbers [5-13].

A recursive algorithm for computing multivariate intersection numbers was proposed in [14] and later refined and applied to a few paradigmatic cases of Feynman integral decomposition [3]. This recursive algorithm was developed in order to compute intersection numbers for twisted cohomologies associated to $n$-forms, which in the general case may contain poles that are not necessarily simple. In the case of logarithmic (dlog) differential forms, owing to the presence of simple poles only, the computation of the intersection numbers is known to be simpler [6, 12].

Recent complementary work $[15,16]$ shows that intersection numbers play a fundamental role in the definition of a diagrammatic coaction for MIs, which combined with the master integral decomposition studied in this paper, as well as in refs. [1-3] paves a way towards comprehensive computations of scattering amplitudes using the tools of intersection theory.

The intersection theory-based decomposition has also been recently applied to the study of Feynman integrals in $d=4 \pm 2 \epsilon$ space-time dimensions, from which an unexpected relation between the behaviors around $\epsilon \rightarrow 0$ and $\epsilon \rightarrow \infty$ emerged [17] and was used to investigate the properties of canonical systems of differential equations [18]. A further 
interesting step for the construction of canonical integrals with intersection theory has been reported in [19]. Moreover, it was observed that using recursion relations for computing intersection numbers can be further refined by relating them to dlog forms at each step of the recursive algorithm [20]. Other recent intersection-theory approaches include [21-23].

This work can be considered as an extension of [3], which contained the essential mathematical details that brought us to the formulation of a decomposition algorithm based on multivariate intersection numbers. In the current work, we address more extensively the problem of evaluating intersection numbers for multivariate forms, providing an explicit description of the 2-form case, and showing their application to the complete decomposition of Feynman integrals in terms of MIs. We show how intersection numbers can be used to establish linear and quadratic relations for Feynman integrals, and, more generally, for AomotoGel'fand generalized hypergeometric functions. The former set of relations yields results that are equivalent to the known integration-by-parts identities (IBPs) [24], while the latter allow for a systematic classification of relations which, for certain type of integrals were originally detected within the application of number-theoretic methods to Feynman integrals, giving rise to interesting conjectures [25-29], proven to be true quite recently [30, 31]. A special set of quadratic relations have been presented in [32], and it would be interesting to investigate if they can be classified as Twisted Riemann Period Relations [4].

In particular, we focus on different ways of using intersection theory in order to derive linear relations for Feynman integrals, as well as the systems of differential equations and the finite difference equations they obey. Moreover, we present here for the first time, a novel algorithm for Feynman integral decomposition, which we will refer to as top-down decomposition, showing that the coefficients of MIs can be suitably extracted by projections via intersection numbers within an iterative strategy, starting from the integrals that correspond to graphs with the highest number of internal lines, and ending with those corresponding to graphs with the lowest possible number of internal lines (given, in the general case, by the product of as many tadpoles as the number of loops).

Following [2,3], we also make use of two other algorithms: the bottom-up decomposition and straight decomposition to similar aim. All these strategies combine the advantages of the integrand decomposition techniques [33-40], the unitarity-based methods [41-55], and the intersections theory-based decomposition.

This work constitutes an important step forward towards the development of a complete algorithm for the decomposition of Feynman integral by means of intersection theory based concepts. In particular, the use of intersection-numbers within the top-down decomposition enhances the effectiveness of the unitarity-based decomposition and of the integrand-decomposition. In fact, on the one side, the applicability of generalised-unitarity for the direct extraction of integral coefficients is known to be limited by two factors: the lack of (complex) integration techniques for evaluating phase-space integrals corresponding to generic generalised cuts (multiple-cut techniques are indeed available only for a limited set of cuts, and mainly at one-loop); the lack of systematic criteria for disentangling the coefficients of master integrals that belong to the same sector on the maximal-cut, i.e. sharing a common set of cut-denominators (usually occurring in dealing with diagrams with more than one loop). On the other side, the integrand decomposition techniques, 
which implement generalised unitarity at the integrand level, provide a decomposition in terms of a set of integrals which is not minimal: in fact, it is known that these integrals can be further reduced to a minimal basis of master integrals by means of IBPs. Using intersection numbers those problems are bypassed, and integrals can be expressed in terms of a minimal set of master integrals.

As originally defined [4], intersection theory for twisted cohomologies and the evaluation of multivariate intersection numbers are applicable to differential forms obeying certain genericity conditions, whose purpose is to regulate boundaries of integration and ensure that they integrate to analytic functions. In the physics language, this corresponds to the analytic regularization of Feynman integrals [56]. To simplify computations, we employ this regularization whenever necessary. It has the additional benefit of resolving the ambiguities that arise when there is a non-trivial overlap between critical points and singularities $[2,20,57]$. Recent mathematical developments, employing the notion of intersection numbers for the relative twisted cohomology [58], seem to offer the possibility of studying the vector space properties of Aomoto-Gel'fand hypergeometric integrals in absence of analytic regulators. This creates a natural path for further investigations of the connections between intersection theory and Feynman integrals, which are left for the future.

The paper is organized as follows: in section 2 we begin by recalling the basics of the Feynman integrals in terms of twisted de Rham (co)homologies and their intersection theory. We show the representation of both the integral and its dual, together with the master decomposition formula needed for their direct decomposition. We discuss different ways to compute the dimension of the cohomology group. The differential equations satisfied by the forms and the dual forms are also provided. Then follows section 3 in which we discuss multivariate intersection numbers. We start with an explicit construction of the 2-variable intersection numbers, which is expressed in terms of the univariate ones recursively. This procedure is generalized, resulting in the final formula for the $n$-variable intersection numbers. We also present an explicit example showing all the steps of the computation of a specific 2-variable intersection number, and discuss a few properties satisfied by the intersection numbers, as well as the simplified formula valid in the case of dlog forms. In section 4, we discuss strategies for the decomposition of an arbitrary Feynman integral. Specifically, we show three different approaches, namely the straight decomposition, the bottom-up decomposition, and the top-down decomposition. Section 5 is dedicated to examples. We first consider the one-loop massless box and perform the decomposition with all these three approaches to show the steps involved explicitly. Moreover, we show the decomposition for the QED triangle as well as the differential equation for the QED sunrise. After that, we provide a few tables with all the key ingredients necessary for the computation of the multivariate intersection numbers needed to obtain the direct decomposition, as well as their differential equations, for the cases of the 1-loop box with 4 different masses, the 2-loop sunrise with 3 different masses, the 2-loop planar and non-planar massless triangle-boxes, as well as 2-loop massless double-box on a triple cut. Finally, section 6 contains our conclusions and discussion. The paper ends with appendix $\mathrm{C}$ containing the explicit forms of the multivariate intersection numbers used for the 1-loop massless box, the QED triangle, and the QED sunrise. 


\section{Feynman integrals and differential forms}

We consider Aomoto-Gel'fand generalized hypergeometric integrals of the form

$$
I=\int_{\mathcal{C}_{R}} u(\mathbf{z}) \varphi_{L}(\mathbf{z})
$$

where $u(\mathbf{z})$ is a multivalued function, $u(\mathbf{z})=\mathcal{B}(\mathbf{z})^{\gamma}$ (or $\left.u(\mathbf{z})=\prod_{i} \mathcal{B}_{i}(\mathbf{z})^{\gamma_{i}}\right)$. In the context of the Feynman integrals addressed in this manuscript $\mathcal{B}$ is the Baikov (graph) polynomial, which has the property that it vanishes on the boundary of the integration domain in (2.1)

$$
\mathcal{B}\left(\partial \mathcal{C}_{R}\right)=0
$$

while $\gamma$ depends on the space-time dimensionality $d$, and on the number of loops and external legs. We assume $\gamma$ to not be an integer, $\gamma \notin \mathbb{Z}$, which follows from dimensional regularization.

On the other hand, $\varphi(\mathbf{z})$ is a single valued differential form

$$
\varphi_{L}(\mathbf{z})=\hat{\varphi}_{L}(\mathbf{z}) d^{n} \mathbf{z}, \quad \hat{\varphi}_{L}(\mathbf{z})=\frac{f(\mathbf{z})}{z_{1}^{a_{1}} \ldots z_{n}^{a_{n}}},
$$

where $\hat{\varphi}_{L}(\mathbf{z})$ denotes its differential-stripped version, $f(\mathbf{z})$ is a rational function and $a_{i}$ are integer exponents, $a_{i} \in \mathbb{Z}$.

One of the key assumptions is that all the poles present in $\varphi_{L}$ must be regulated by $u(\mathbf{z})$. In genuine Feynman integrals this assumption is often violated; in this work we present two different strategies for overcoming this apparent obstacle.

It is possible to identify equivalence classes of differential $n$-forms entering the integral (2.1). Forms in the same class are those that differ by a covariant derivative and give the same result upon integration, as will be explained below.

\subsection{The cohomology group and its dual}

Consider an $(n-1)$-differential form $\xi_{L}$. In the absence of boundary terms due to $(2.2)$ we have:

$$
0=\int_{\mathcal{C}_{R}} d\left(u \xi_{L}\right)=\int_{\mathcal{C}_{R}}\left(d u \wedge \xi_{L}+u d \xi_{L}\right)=\int_{\mathcal{C}_{R}} u\left(\frac{d u}{u} \wedge+d\right) \xi_{L}=\int_{\mathcal{C}_{R}} u \nabla_{\omega} \xi_{L}
$$

where

$$
\nabla_{\omega}=d+\omega \wedge, \quad \omega=d \log u
$$

Thus we can write

$$
\int_{\mathcal{C}_{R}} u \varphi_{L}=\int_{\mathcal{C}_{R}} u\left(\varphi_{L}+\nabla_{\omega} \xi_{L}\right)
$$

The forms $\varphi_{L}$ and $\varphi_{L}+\nabla_{\omega} \xi_{L}$, which give the same result upon integration, are in the same equivalence class

$$
\varphi_{L} \sim \varphi_{L}+\nabla_{\omega} \xi_{L}
$$

Differential $n$-forms modulo the equivalence relation (2.7) belong to a vector space, the twisted cohomology group $H_{\omega}^{n}$, and elements in this vector space are denoted by $\left\langle\varphi_{L}\right|$. 
In a similar way one can define an equivalence relation among integration contours which give the same result upon integration. Integration contours modulo the equivalence relation, are denoted by $\left.\mid \mathcal{C}_{R}\right]$ and belong to the vector space $H_{n}^{\omega}$, referred to as the twisted homology group.

The integral of eq. (2.1) can be regarded as a paring between $\left\langle\varphi_{L}\right|$ and the function $u(\mathbf{z})$, integrated over the contour $\left.\mid \mathcal{C}_{R}\right]$

$$
\left.I=\int_{\mathcal{C}_{R}} u(\mathbf{z}) \varphi_{L}(\mathbf{z})=\left\langle\varphi_{L}\right| \mathcal{C}_{R}\right] .
$$

Given this terminology, we may now define a dual integral, given by

$$
\tilde{I}=\int_{\mathcal{C}_{L}} u(\mathbf{z})^{-1} \varphi_{R}(\mathbf{z})
$$

and consider the covariant derivative

$$
\nabla_{-\omega}=d-\omega \wedge, \quad \omega=d \log u .
$$

In analogy to (2.7) we can derive the equivalence relation

$$
\varphi_{R} \sim \varphi_{R}+\nabla_{-\omega} \xi_{R}
$$

such that differential $n$-forms modulo the equivalence relation eq. (2.11) belong to the dual vector space $\left(H_{\omega}^{n}\right)^{*}=H_{-\omega}^{n}$; the elements of this space are denoted by $\left|\varphi_{R}\right\rangle$. As done above, one can also consider an equivalence relation among integration contours, which leads to the vector space $\left(H_{n}^{\omega}\right)^{*}=H_{n}^{-\omega}$ whose elements are denoted by $\left[\mathcal{C}_{L} \mid\right.$.

The dual integral of eq. (2.9) is interpreted as paring between $\left|\varphi_{R}\right\rangle$ and the function $u(\mathbf{z})^{-1}$, integrated over the contour $\left[\mathcal{C}_{L} \mid\right.$

$$
\tilde{I}=\left[\mathcal{C}_{L}\left|\varphi_{R}\right\rangle\right.
$$

Aomoto-Gel'fand (AG) integrals are known to obey Gauss contiguity relations. Similarly Feynman integrals obey linear relations, dubbed integration by parts identities [24]. Those identities can be used to identify a minimal set of functions which constitute a basis that generates a vector space [3], which - by borrowing the terminology from Feynman multi-loop calculus - we will refer to as master integrals (MIs). Linear relations among integrals can therefore be used to decompose any AG/Feynman integral in terms of MIs, as well as to derive (systems of first order) difference and differential equations for MIs.

Let us observe that dual integrals are AG integrals where $u^{-1}$ appears in the integrand (instead of $u$ ). In the case of dimensionally regulated Feynman integrals, $u$ consists of graph polynomials raised to a non-integer power that depends on the space-time dimensions $d$. Therefore, dual integrals represent integrals in a different dimension (for which the exponents of the graph polynomials becomes the opposite of the one contained in $u$ ).

Linear relations for Feynman integrals can be derived by projections using intersection numbers, purely algebraically, in the same way as any vector admits a decomposition in terms of a basis, within a vector space. Intersection theory for twisted de Rham (co)homology provides the mathematical framework of a vector space structure, characterized by its dimension, its bases and its scalar product, which we present in the following. 


\subsection{Dimension of twisted cohomology groups}

In ref. [57], the number of MIs within the IBP-decomposition was related to the number of independent contours of integration, generating no surface terms. The condition in eq. (2.2) relates the geometric properties of the multivariate polynomial $\mathcal{B}$ to the analytic properties derived from the integration domain. In particular, using a correspondence between the basis cycles and the critical points of the graph-polynomial of the considered integral parametrization, the number of MIs was related to the rank of the homology groups $H_{n}^{ \pm \omega}$.

In refs. [1-3], we considered a dual, equivalent description of the same problem, in terms of independent differential forms. Accordingly, we define $\nu$ as the dimension of the twisted cohomology group, respectively, $H_{ \pm \omega}^{n}$, here considered as a vector space,

$$
\nu=\operatorname{dim} H_{ \pm \omega}^{n} .
$$

The complex Morse (Picard-Lefschetz) theory allows us to determine $\nu$ as the number of critical points of the function $\log u(\mathbf{z})$ [57]. We define

$$
\omega=d \log u(\mathbf{z})=\sum_{i=1}^{n} \hat{\omega}_{i} d z_{i}
$$

and the number of critical points is given by the number of solutions of the (zero dimensional) system

$$
\hat{\omega}_{i} \equiv \partial_{z_{i}} \log u(\mathbf{z})=0, \quad i=1, \ldots, n .
$$

The number of solutions of (2.15) can be determined without computing explicitly its zeros [57]. In our applications the function $u(\mathbf{z})$ always takes the form $u(\mathbf{z})=\prod_{j} \mathcal{B}_{j}^{\gamma_{j}}(\mathbf{z})$, which gives the equations:

$$
\hat{\omega}_{i}=\sum_{j} \gamma_{j} \frac{\partial_{z_{i}} \mathcal{B}_{j}}{\mathcal{B}_{j}}, \quad i=1, \ldots, n
$$

In the absence of critical points at infinity, the number of solutions of (2.15) equals to the dimension of the quotient space for the ideal ${ }^{1}$

$$
\mathcal{I}=\left\langle\beta_{1}, \ldots, \beta_{n}, z_{0} \prod_{j} \mathcal{B}_{j}-1\right\rangle \quad \text { with } \quad \beta_{k} \equiv \sum_{i} \gamma_{i}\left(\partial_{z_{k}} \mathcal{B}_{i}\right) \prod_{j \neq i} \mathcal{B}_{j}
$$

In the special case when $u(\mathbf{z})=\mathcal{B}^{\gamma}(\mathbf{z})$, it becomes simply [57]

$$
\mathcal{I}=\left\langle\partial_{z_{1}} \mathcal{B}, \ldots, \partial_{z_{n}} \mathcal{B}, z_{0} \mathcal{B}-1\right\rangle
$$

Considering a Gröbner basis $\mathcal{G}$ generating $\mathcal{I}$, the Shape Lemma (see, e.g. [59], and [38] for an application to physics) ensures that the number $\nu$ of zeros of $\mathcal{I}$, and hence the number of the solutions of the system (2.15), is the dimension of the quotient ring,

$$
\nu=\operatorname{dim}(\mathbb{C}[\mathbf{z}] /\langle\mathcal{G}\rangle),
$$

\footnotetext{
${ }^{1}$ We introduce an extra variable $z_{0}$ in order to prevent the case when $\mathcal{B}_{j}=0$ for either $j$.
} 
where $\mathbb{C}[\mathbf{z}]$ is the set of all polynomials that vanish on the zeroes of $\mathcal{I}$ (they identify a discrete variety, $V \subset \mathbb{C}^{\nu}$ ). In particular, the lemma ensures that the degree of the remainder of the polynomial division modulo $\mathcal{G}$ is $\nu+1$.

In ref. [3], we recalled that $\nu$ can be computed using one of the many ways of evaluating the topological Euler characteristic $\chi(X): X=\mathbb{C P}^{n}-\mathcal{P}_{\omega}$, where $\mathcal{P}_{\omega} \equiv$ set of poles of $\left.\omega\right\}$ in projective space. This relation can be written as

$$
\nu=|\chi(X)|=(-1)^{n}\left(n+1-\chi\left(\mathcal{P}_{\omega}\right)\right),
$$

where we used $\chi\left(\mathbb{C P}^{n}\right)=n+1$ together with the inclusion-exclusion principle for Euler characteristics. In other words, to compute $\nu$, it is sufficient to evaluate $\chi\left(\mathcal{P}_{\omega}\right)$ of the projective variety $\mathcal{P}_{\omega}$ (see also refs. [60-62]).

In the following, we will compute the dimension of the cohomology groups to determine the size of the basis of differential forms for different choices of $H_{ \pm \omega}^{n}$, each characterized by $\omega$, or correspondingly by $u$.

\subsection{Intersection numbers for twisted (co)homology classes}

Within twisted de Rham theory, $\left\langle\varphi_{L}\right|$ and $\left|\varphi_{R}\right\rangle$ are elements of the twisted cohomology class $H_{\omega}^{n}$ and the dual cohomology class $H_{-\omega}^{n}$ respectively. Because of a duality between twisted cycles and co-cycles [63], $\left[\mathcal{C}_{L} \mid\right.$ and $\left.\mid \mathcal{C}_{R}\right]$ can be considered as elements of the homology class $H_{n}^{\omega}$ and the dual homology class $H_{n}^{-\omega}$. Beside the two type of pairings that defined the integrals and the dual integrals, respectively $\left.\left\langle\varphi_{L}\right| \mathcal{C}_{R}\right]$ and $\left[\mathcal{C}_{L}\left|\varphi_{R}\right\rangle\right.$, defined above, one can consider:

- intersection numbers of twisted cycles $\left[\mathcal{C}_{L} \mid \mathcal{C}_{R}\right]$, as introduced in [64];

- intersection numbers of twisted co-cycles $\left\langle\varphi_{L} \mid \varphi_{R}\right\rangle$, which were first considered in [4].

While we refer the interested reader to consult the original publications on the topics, we will briefly review some properties of intersection numbers for twisted co-cycles (here refereed to also as twisted forms, or simply $n$-forms), which are relevant to our later discussion.

Given the integrals $\left.I=\left\langle\varphi_{L}\right| \mathcal{C}_{R}\right]$ and $\tilde{I}=\left[\mathcal{C}_{L}\left|\varphi_{R}\right\rangle\right.$, we define the intersection number between the corresponding $n$-forms, $\varphi_{L}=\hat{\varphi}_{L} d z_{1} \wedge \ldots \wedge d z_{n}$ and $\varphi_{R}=\hat{\varphi}_{R} d z_{1} \wedge \ldots \wedge d z_{n}$ as

$$
\left\langle\varphi_{L} \mid \varphi_{R}\right\rangle=\frac{1}{(2 \pi i)^{n}} \int_{X} \varphi_{L} \wedge \varphi_{R}
$$

In the general case, the integral over $X$ can be performed by iteration, and applying Stokes' theorem one variable at a time (namely, by splitting it into one-dimensional fibers), it reduces to a nested sequence of contour integrations, performed by Cauchy's residue theorem, as it will be shown later (see also section 3.2 of [65]). As required by the proper mathematical definition of the intersection number for twisted cohomology, we assume that $\varphi_{L}$ and $\varphi_{R}$ have compact support near the boundary of $X$ and that, until differently specified, they have poles which are regulated by the multi-valued function $u$. 
Two interesting properties of intersection numbers play a role in devising the decomposition algorithm we propose:

- Intersection numbers are invariant under a change of differential forms within the same equivalence classes, namely

$$
\left\langle\varphi_{L} \mid \varphi_{R}\right\rangle=\left\langle\varphi_{L}^{\prime} \mid \varphi_{R}\right\rangle=\left\langle\varphi_{L} \mid \varphi_{R}^{\prime}\right\rangle=\left\langle\varphi_{L}^{\prime} \mid \varphi_{R}^{\prime}\right\rangle
$$

where

$$
\begin{gathered}
\varphi_{L}^{\prime}=\varphi_{L}+\nabla_{\omega} \xi_{L}, \\
\varphi_{R}^{\prime}=\varphi_{R}+\nabla_{-\omega} \xi_{R},
\end{gathered}
$$

and the covariant derivatives $\nabla_{ \pm \omega}$ defined in eqs. (2.5) and (2.10), explicitly read

$$
\nabla_{ \pm \omega}=\sum_{i=1}^{n} d z_{i}\left(\partial_{z_{i}} \pm \hat{\omega}_{i}\right) \wedge
$$

while $\xi_{L}$ and $\xi_{R}$ are arbitrary $(n-1)$-forms with poles regulated by $u$.

The invariance of intersection numbers under the replacement of forms belonging to the same equivalence class can be useful: $i$ ) for substituting differential forms having higher poles with (equivalent) forms that have simple poles [23], as it will be recalled in section 3.6 ; ii) for substituting differential forms having poles that are not regulated with (equivalent) forms that are fully regulated, as it will be shown in section 5.1.3.

- Intersection numbers obey the symmetry relation

$$
\left\langle\varphi_{L} \mid \varphi_{R}\right\rangle_{\omega}=(-1)^{n}\left\langle\varphi_{R} \mid \varphi_{L}\right\rangle_{-\omega}
$$

which follows directly from the definition and the fact that commuting $\varphi_{L}$ with $\varphi_{R}$ yields a sign change of $(-1)^{n}$. We stress that the right-hand side is evaluated with respect to $-\omega$ rather than $\omega$.

Before providing the details for the evaluation of intersection numbers, which are going to be presented in section 3, let us recall their main applications: the derivation of linear and quadratic relations and of systems of differential equations for AG/Feynman Integrals. They can be presented in full generality, purely algebraically, without any specific reference to the number of integration variable and to the explicit computation of intersection numbers.

\subsection{Linear and quadratic relations}

The reduction of a given integral, $\left.I=\left\langle\varphi_{L}\right| \mathcal{C}_{R}\right]$, in terms of a set of $\nu$ MIs, $\left.J_{i}=\left\langle e_{i}\right| \mathcal{C}_{R}\right]$

$$
I=\sum_{i=1}^{\nu} c_{i} J_{i}
$$


can be interpreted in terms of differential forms, as

$$
\left\langle\varphi_{L}\right|=\sum_{i=1}^{\nu} c_{i}\left\langle e_{i}\right|
$$

since the integration cycle is the same for all the integrals of eq. (2.27). Likewise, the decomposition of a dual integral $\tilde{I}=\left[\mathcal{C}_{L}\left|\varphi_{R}\right\rangle\right.$ in terms of a set of $\nu$ dual MIs $\tilde{J}_{i}=\left[\mathcal{C}_{L}\left|h_{i}\right\rangle\right.$

$$
\tilde{I}=\sum_{i=1}^{\nu} \tilde{c}_{i} \tilde{J}_{i}
$$

becomes

$$
\left|\varphi_{R}\right\rangle=\sum_{i=1}^{\nu} \tilde{c}_{i}\left|h_{i}\right\rangle
$$

The coefficients $c_{i}$, and $\tilde{c}_{i}$ in eqs. (2.28), (2.30) are determined by the master decomposition formulas $[1,2]$

$$
\begin{aligned}
c_{i} & =\sum_{j=1}^{\nu}\left\langle\varphi_{L} \mid h_{j}\right\rangle\left(\mathbf{C}^{-1}\right)_{j i}, \\
\tilde{c}_{i} & =\sum_{j=1}^{\nu}\left(\mathbf{C}^{-1}\right)_{i j}\left\langle e_{j} \mid \varphi_{R}\right\rangle,
\end{aligned}
$$

where we introduced the (inverse of the) metric matrix

$$
\mathbf{C}_{i j}=\left\langle e_{i} \mid h_{j}\right\rangle .
$$

In the above formula $\mathbf{C}$ is a $(\nu \times \nu)$-matrices of intersection numbers of basic forms $\left\langle e_{i}\right|$ and dual-forms $\left|h_{i}\right\rangle$, which, in general, differs from the identity matrix, but, for suitably chosen bases can reduce to it, hence simplifying eqs. (2.31), (2.32). The formal derivation of the latter two equations are given in appendix $\mathrm{A}$.

By substituting eq. (2.31) in eq. (2.28) (or eq. (2.32) in eq. (2.30)), we obtain a representation of the identity operator in the cohomology space

$$
\sum_{i, j=1}^{\nu}\left|h_{i}\right\rangle\left(\mathbf{C}^{-1}\right)_{i j}\left\langle e_{j}\right|=\mathbb{I}_{c}
$$

Similarly, in the homology space, the resolution of the identity is

$$
\left.\sum_{i, j=1}^{\nu} \mid \mathcal{C}_{R, i}\right]\left(\mathbf{H}^{-1}\right)_{i j}\left[\mathcal{C}_{L, j} \mid=\mathbb{I}_{h},\right.
$$

where $\mathbf{H}_{i j}=\left[\mathcal{C}_{L, i} \mid \mathcal{C}_{R, j}\right]$ is the metric matrix for the twisted cycles. The operators $\mathbb{I}_{c}$ and $\mathbb{I}_{h}$ can be inserted either in the bilinear pairing between the twisted cocyles or the twisted cycles, to obtain the quadratic identities

$$
\begin{aligned}
\left\langle\varphi_{L} \mid \varphi_{R}\right\rangle & \left.=\sum_{i, j=1}^{\nu}\left\langle\varphi_{L}\right| \mathcal{C}_{R, i}\right]\left(\mathbf{H}^{-1}\right)_{i j}\left[\mathcal{C}_{L, j}\left|\varphi_{R}\right\rangle\right. \\
{\left[\mathcal{C}_{L} \mid \mathcal{C}_{R}\right] } & =\sum_{i, j=1}^{\nu}\left[\mathcal{C}_{L}\left|h_{i}\right\rangle\left(\mathbf{C}^{-1}\right)_{i j}\left\langle e_{j}\right| \mathcal{C}_{R}\right]
\end{aligned}
$$


which are known as Twisted Riemann's Period Relations (TRPR) [4]. TRPR relates intersection numbers for (co)-homologies to products of integrals and dual integrals.

Let us emphasize that the coefficients $c_{i}$ in eq. (2.31) are independent of the choice of the dual basis $\left|h_{j}\right\rangle$. A suitable choice of the dual basis may simplify the intermediate steps of the evaluation, which requires the separate calculations of the intersection numbers $\left\langle\varphi_{L} \mid h_{j}\right\rangle$ and $\left\langle e_{i} \mid h_{j}\right\rangle$. Similar considerations hold for $\tilde{c}_{i}$ in eq. (2.32), which are independent of $\left.\left\langle e_{i}\right|\right)$. Since the master decomposition formula eq. (2.31) involves the inverse of the matrix C, further simplifications arise when it is close to a diagonal matrix, hence implying that $\left|h_{j}\right\rangle$ and $\left\langle e_{i}\right|$ are as othogonal as possible. The construction of orthonormal bases of forms can be achieved by the Gram-Schmidt algorithm, using the intersection numbers as scalar products. In the case of 1 -form, orthonormal bases can be built directly, simply using the expression of $\omega[2]$.

Recent mathematical literature on intersection numbers of twisted cycles and co-cycles include application to Gel'fand-Kapranov-Zelevinski systems [13, 66, 67] and to quadratic relations [25-31].

\subsection{Differential equation for forms and dual forms}

The decomposition of differential forms in terms of master forms, implemented by the use of eqs. (2.31), (2.32), yields the direct derivation of the systems of differential equations [3, 14]. In particular, the basis $\left\langle e_{i}\right|$ and the dual basis $\left|h_{i}\right\rangle$ obey a system of first order differential equations, with respect to any external variable, say $x$, respectively reading as,

$$
\begin{aligned}
\partial_{x}\left\langle e_{i}\right| & =\boldsymbol{\Omega}_{i j}\left\langle e_{j}\right|, \\
\partial_{x}\left|h_{i}\right\rangle & =-\left|h_{j}\right\rangle \widetilde{\boldsymbol{\Omega}}_{j i} .
\end{aligned}
$$

The matrices $\boldsymbol{\Omega}$ and $\widetilde{\boldsymbol{\Omega}}$ arise from the decompositions,

$$
\begin{aligned}
& \partial_{x}\left\langle e_{i}\right|=\left\langle\left(\partial_{x}+\sigma\right) e_{i}\right|=\underbrace{\left\langle\left(\partial_{x}+\sigma \wedge\right) e_{i} \mid h_{k}\right\rangle\left(\mathbf{C}^{-1}\right)_{k j}}_{\mathbf{\Omega}_{i j}}\left\langle e_{j}\right|, \\
& \partial_{x}\left|h_{i}\right\rangle=\left|\left(\partial_{x}-\sigma\right) h_{i}\right\rangle=\left|h_{j}\right\rangle \underbrace{\left(\mathbf{C}^{-1}\right)_{j k}\left\langle e_{k} \mid\left(\partial_{x}-\sigma \wedge\right) h_{i}\right\rangle}_{-\widetilde{\boldsymbol{\Omega}}_{j i}},
\end{aligned}
$$

where $\sigma \equiv \partial_{x} \log u$. Let us observe that the combinations $\partial_{x} \pm \sigma \wedge \equiv \nabla_{x, \pm \sigma}$ may be also interpreted as covariant derivatives. The systems of differential equations for forms directly translates into systems of differential equations for MIs, as follows,

$$
\partial_{x} J_{i}=\boldsymbol{\Omega}_{i j} J_{j}, \quad \partial_{x} \tilde{J}_{i}=-\tilde{\boldsymbol{\Omega}}_{i j} \tilde{J}_{j}, \quad i, j=1, \ldots, \nu .
$$

We observe that although the integration domain of $J_{i}$ and $\tilde{J}_{i}$, respectively $\mathcal{C}_{R}$ and $\mathcal{C}_{L}$, may depend on the $x$ variable, the condition that $u$ vanishes at the integration boundaries, $u\left(\partial \mathcal{C}_{R, L}\right)=0$, preserves the commutation between the $x$-differentiation and integration. For the case of Feynman integrals, $\boldsymbol{\Omega}$ and $\widetilde{\boldsymbol{\Omega}}$ on the space-time dimension $d$ and on kinematic variables, including $x$. Quite generally, for Aomoto-Gel'fand integrals, these matrices depend on the external variables and on the parameters appearing in the definition of $u$. 
Using the above formulas, one can relate the matrices $\boldsymbol{\Omega}$ and $\widetilde{\boldsymbol{\Omega}}$ through the identity

$$
\partial_{x}\left\langle e_{i} \mid h_{j}\right\rangle=\left(\partial_{x}\left\langle e_{i}\right|\right)\left|h_{j}\right\rangle+\left\langle e_{i}\right|\left(\partial_{x}\left|h_{j}\right\rangle\right)=\boldsymbol{\Omega}_{i k}\left\langle e_{k} \mid h_{j}\right\rangle-\left\langle e_{i} \mid h_{k}\right\rangle \widetilde{\boldsymbol{\Omega}}_{k j},
$$

which, in matrix notation, reads as,

$$
\partial_{x} \mathbf{C}=\mathbf{\Omega} \mathbf{C}-\mathbf{C} \widetilde{\boldsymbol{\Omega}} .
$$

In particular, for orthonormal bases, $\mathbf{C}=\mathbb{I}$, therefore $\boldsymbol{\Omega}=\widetilde{\boldsymbol{\Omega}}$.

Intersection theory has been recently used to identify special bases of Feynman integrals admitting canonical systems of differential equations [18] (see also [68]), according to the structure of $u[19]$.

\section{Multivariate intersection numbers}

Multivariate intersection numbers constitute the key operation for generating linear and quadratic relations among integrals and dual integrals. In particular, they enter the decomposition of differential forms in terms of a set of master forms, therefore of the corresponding integrals in terms of master integrals, according to the master decomposition formulas eq. (2.31), (2.32). It is important to observe that these decomposition formulas hold for generic $n$-forms. Therefore, algorithms for the evaluation of intersection numbers play an important role in the development of novel strategies for computing scattering amplitudes in Physics as well as for deriving relations among transcendental functions in Mathematics.

\subsection{Intersection numbers of logarithmic forms}

Intersection numbers for multivariate logarithmic forms were considered in $[6,12,14]$. In particular, if $\varphi_{L}$ and $\varphi_{R}$ are both logarithmic differential forms (dlog forms), and $\omega_{i}$ have simple poles, the intersection numbers can be evaluated as:

$$
\left\langle\varphi_{L} \mid \varphi_{R}\right\rangle=\left.(-1)^{n} \sum_{\left(z_{1}^{*}, \ldots, z_{n}^{*}\right)} \operatorname{det}^{-1}\left[\begin{array}{ccc}
\partial_{z_{1}} \hat{\omega}_{1} & \ldots & \partial_{z_{n}} \hat{\omega}_{1} \\
\vdots & \ddots & \vdots \\
\partial_{z_{1}} \hat{\omega}_{n} & \ldots & \partial_{z_{n}} \hat{\omega}_{n}
\end{array}\right] \widehat{\varphi}_{L} \widehat{\varphi}_{R}\right|_{\left(z_{1}, \ldots, z_{n}\right)=\left(z_{1}^{*}, \ldots z_{n}^{*}\right)}
$$

where the sum goes over all the $\nu$ critical points, identified with the $n$-ples $\left(z_{1}^{*}, \ldots, z_{n}^{*}\right)$ that solve the system of equations

$$
\hat{\omega}_{i} \equiv \partial_{z_{i}} \log u(\mathbf{z})=0, \quad i=1, \ldots, n,
$$

as in eq. (2.15). When at least one of the forms is non-logarithmic, the formula (3.1) is only valid asymptotically in the limit $\gamma \rightarrow \infty$. In those cases, one can still calculate intersection numbers making use of the above formula within a series expansion in $1 / \gamma$, as it was successfully applied to the computation of differential equations for certain Feynman integrals in [17]. 


\subsection{Intersection numbers of general forms}

Logarithmic differential forms have been subject of intense mathematical developments. Nonetheless, generic Feynman integrals may correspond to pairing of forms that are not necessarily logarithmic, and therefore it becomes necessary to devise algorithms for computation of intersection numbers for general rational forms.

The evaluation of intersection numbers of multivariate differential forms has been introduced in [14] and systematized in [3] for the derivation of linear relations of Feynman integrals as well as of hypergeometric functions, by adopting an iterative procedure. According to this approach, the calculation of the intersection number of two $n$-forms proceeds recursively, in terms of the intersection numbers of $(n-1)$-forms, until reaching the terminating condition, given by the univariate intersection numbers $[1,2]$.

One of the goals of this work is to provide a pedagogical introduction to the evaluation of multivariate intersection numbers (for twisted de Rham cohomology) by means of the recursive algorithm. To this aim, let us consider integrals with $n$ integration variables $\left\{z_{i_{1}}, \ldots, z_{i_{n}}\right\}$, which can be seen as iterative integrals, with a nested structure that follows from the chosen ordering $\left\{i_{1}, \ldots, i_{k}\right\}$ of the integers $\{1, \ldots, n\}$. In order to compute multivariate intersection numbers for differential $k$-forms, we need to compute the dimension of the cohomology groups for all differential $k$-forms, from $k=1$ to $k=n$. They can be obtained, for instance, by counting the number $\nu_{\mathbf{k}}$ of solutions of the system of equations given by eq. (2.15),

$$
\hat{\omega}_{j} \equiv \partial_{z_{j}} \log u(\mathbf{z})=0, \quad j=i_{1}, \ldots, i_{k},
$$

where $\mathbf{k}=\left\{i_{1}, \ldots, i_{k}\right\}$ is a subset of $\{1, \ldots, n\}$ with $k$ distinct elements. In this way, one obtains a list of dimensions

$$
\nu_{\mathbf{1}}, \nu_{\mathbf{2}}, \ldots, \nu_{\mathbf{n}}, \quad \text { with }, \quad \mathbf{1}=\left\{i_{1}\right\}, \quad \mathbf{2}=\left\{i_{1}, i_{2}\right\}, \quad \ldots, \quad \mathbf{n}=\left\{i_{1}, i_{2}, \ldots, i_{n}\right\},
$$

corresponding to the number of master integrals within each step of the iterative integration (respectively in $\left\{z_{i_{1}}\right\}$, in $\left\{z_{i_{1}}, z_{i_{2}}\right\}, \ldots$, in $\left\{z_{i_{1}}, \ldots, z_{i_{n}}\right\}$ ).

It is interesting to observe that $\nu_{\mathbf{n}}$ is trivially independent of the ordering of the integration variables. On the other hand, the sequence of the dimensions of all the subspaces $\nu_{\mathbf{k}}$ may indeed change according to the chosen permutation of $\{1,2, \ldots, n\}$ that correspond to the ordering of nested integrations. As a working principle, we choose the ordering that minimizes the sizes of $\nu_{\mathbf{k}}$ for all $k$-forms $(k=1, \ldots, n)$.

\subsection{Intersection numbers for 1-forms}

Let us briefly recall the intersection number for 1-forms [4], discussed at length in [1, 2]. Consider a generic integral with one integration variable,

$$
\left.I=\int_{\mathcal{C}_{R}^{(1)}} \varphi_{L}^{(\mathbf{1})}\left(z_{1}\right) u\left(z_{1}\right)=\left\langle\varphi_{L}^{(\mathbf{1})}\right| \mathcal{C}_{R}^{(\mathbf{1})}\right],
$$

where $\mathbf{1}=\{1\}$. Similarly, we consider dual integrals of the type,

$$
\tilde{I}=\int_{\mathcal{C}_{L}^{(1)}} \varphi_{R}^{(\mathbf{1})}\left(z_{1}\right) u^{-1}\left(z_{1}\right)=\left[\mathcal{C}_{L}^{(\mathbf{1})}\left|\varphi_{R}^{(\mathbf{1})}\right\rangle .\right.
$$


We compute $\omega_{1}=d \log u\left(z_{1}\right)=\hat{\omega}_{1} d z_{1}$, and determine $\nu_{\mathbf{1}}$ by counting the critical points of $\hat{\omega}_{z_{1}}$. Simultaneously, we define $\mathcal{P}_{\omega_{1}}$ as the sets of its poles (including the pole at $\infty$ ). Then, the intersection number between 1-forms can be computed as,

$$
\left\langle\varphi_{L}^{(\mathbf{1})} \mid \varphi_{R}^{(\mathbf{1})}\right\rangle=\sum_{p \in \mathcal{P}_{\omega_{1}}} \operatorname{Res}_{z_{1}=p}\left[\psi^{(p)} \varphi_{R}^{(\mathbf{1})}\right]
$$

where $\psi^{(p)}$ is the local solution of the differential equation

$$
\nabla_{\omega_{1}} \psi^{(p)}=\varphi_{L}^{(\mathbf{1})}
$$

around the point $z_{1}=p$.

\subsection{Intersection numbers for 2-forms}

We consider instructive to show how the intersection numbers of 2 -forms can be written recursively in terms of intersection numbers of 1 -forms.

Consider an integral with two integration variables $\left\{z_{1}, z_{2}\right\}$, generically written as,

$$
\left.I=\int_{\mathcal{C}_{R}^{(2)}} \varphi_{L}^{(2)}\left(z_{1}, z_{2}\right) u\left(z_{1}, z_{2}\right)=\left\langle\varphi_{L}^{(2)}\right| \mathcal{C}_{R}^{(2)}\right]
$$

where $\mathbf{2}=\{1,2\}, \varphi_{L}^{(\mathbf{2})}$ is a differential 2 -form in the variables $z_{1}$ and $z_{2}$, i.e. $\left\langle\varphi_{L}\right|=$ $\hat{\varphi}_{L}\left(z_{1}, z_{2}\right) d z_{1} \wedge d z_{2}$, while $\mathcal{C}_{R}^{(\mathbf{2})}$ is a two-dimensional integration domain embedded in some ambient space $X$ with complex dimension 2 . We assume ${ }^{2}$ that $X$ admits a fibration into one-dimensional spaces, say $X_{2} \ni z_{2}$ and $X_{1} \ni z_{1}$, yielding the corresponding decompositions of $\varphi_{L}^{(\mathbf{2})}, \mathcal{C}_{R}^{(\mathbf{2})}$. Similarly, we can consider a dual integral, given by

$$
\tilde{I}=\int_{\mathcal{C}_{L}^{(\mathbf{2})}} \varphi_{R}^{(\mathbf{2})}\left(z_{1}, z_{2}\right) u^{-1}\left(z_{1}, z_{2}\right)=\left[\mathcal{C}_{L}^{(\mathbf{2})}\left|\varphi_{R}^{(\mathbf{2})}\right\rangle\right.
$$

Our goal is the evaluation of $\left\langle\varphi_{L}^{(\mathbf{2})} \mid \varphi_{R}^{(\mathbf{2})}\right\rangle$, in terms of intersection numbers for 1-forms.

Given $u\left(z_{1}, z_{2}\right) \equiv u(\mathbf{z})$, we define:

$$
\omega=d \log u(\mathbf{z})=\sum_{i=1}^{2} \hat{\omega}_{i} d z_{i} .
$$

From $\omega$ we determine: the dimension $\nu_{1}$ with $\mathbf{1}=\{1\}$, counting the solutions of $\hat{\omega}_{1}=0$; and the dimension $\nu_{\mathbf{2}}$ with $\mathbf{2}=\{1,2\}$, counting the solutions of the system $\hat{\omega}_{1}=0, \hat{\omega}_{2}=0$. The former number $\nu_{\mathbf{1}}$ corresponds to the number of master 1-forms in $z_{1}$, which correspond to the MIs emerging from the integration in $z_{1}$, while the latter, $\nu_{\mathbf{2}}$, to the number of master 2 -forms in $z_{1}$ and $z_{2}$, therefore to the number of MIs of the integrals $I$ in eq. (3.9).

We can therefore choose the bases of forms $\left\langle e_{i}^{(\mathbf{1})}\right|$ and $\left|h_{i}^{(\mathbf{1})}\right\rangle$ for $i=1, \ldots, \nu_{\mathbf{1}}$, and compute the metric matrix $\mathbf{C}_{(\mathbf{1})}$, i.e. the matrix of intersection numbers,

$$
\left(\mathbf{C}_{(\mathbf{1})}\right)_{i j} \equiv\left\langle e_{i}^{(\mathbf{1})} \mid h_{j}^{(\mathbf{1})}\right\rangle .
$$

\footnotetext{
${ }^{2}$ This does not necessarily mean that $X=X_{2} \times X_{1}$, since $X_{1}=X_{1}\left(z_{2}\right)$ can depend on $z_{2}$ (but $X_{2}$ does not depend on $z_{1}$ ).
} 
We make use of eqs. (2.31), (2.32) to decompose the 2-forms in terms of 1 -forms, by projecting the former on the chosen bases of 1 -forms,

$$
\left\langle\varphi_{L}^{(\mathbf{2})}\left|=\sum_{i=1}^{\nu_{1}}\left\langle e_{i}^{(\mathbf{1})}\left|\wedge\left\langle\varphi_{L, i}^{(2)}|, \quad| \varphi_{R}^{(\mathbf{2})}\right\rangle=\sum_{i=1}^{\nu_{1}}\right| h_{i}^{(\mathbf{1})}\right\rangle \wedge\right| \varphi_{R, i}^{(2)}\right\rangle,
$$

with

$$
\begin{aligned}
\left\langle\varphi_{L, i}^{(2)}\right| & =\left\langle\varphi_{L}^{(\mathbf{2})} \mid h_{j}^{(\mathbf{1})}\right\rangle\left(\mathbf{C}_{(\mathbf{1})}^{-1}\right)_{j i} \\
\left|\varphi_{R, i}^{(2)}\right\rangle & =\left(\mathbf{C}_{(\mathbf{1})}^{-1}\right)_{i j}\left\langle e_{j}^{(\mathbf{1})} \mid \varphi_{R}^{(\mathbf{2})}\right\rangle .
\end{aligned}
$$

To compute the intersection numbers of 2 -forms, we also need the $\left(\nu_{\mathbf{1}} \times \nu_{\mathbf{1}}\right)$-matrix $\boldsymbol{\Omega}^{(2)}$ associated to the system of differential equations in $z_{2}$ obeyed by the bases $\left\langle e_{i}^{(\mathbf{1})}\right|$,

$$
\partial_{z_{2}}\left\langle e_{i}^{(\mathbf{1})}\right| \equiv\left\langle\left(d_{z_{2}}+\omega_{2} \wedge\right) e_{i}^{(\mathbf{1})}\right|=\mathbf{\Omega}_{i j}^{(2)}\left\langle e_{j}^{(\mathbf{1})}\right| .
$$

$\boldsymbol{\Omega}^{(2)}$ is obtained by projecting the form $\left\langle\left(d_{z_{2}}+\omega_{2} \wedge\right) e_{i}^{(\mathbf{1})}\right|$ on the basis $\left\langle e_{i}^{(\mathbf{1})}\right|$, using eq. (2.31),

$$
\mathbf{\Omega}_{i j}^{(2)}=\left\langle\left(d_{z_{2}}+\omega_{2} \wedge\right) e_{i}^{(\mathbf{1})} \mid h_{k}^{(\mathbf{1})}\right\rangle\left(\mathbf{C}_{(\mathbf{1})}^{-1}\right)_{k j} .
$$

The intersection number for 2-forms can be finally computed as [14],

$$
\left\langle\varphi_{L}^{(\mathbf{2})} \mid \varphi_{R}^{(\mathbf{2})}\right\rangle=\sum_{i, j=1}^{\nu_{1}} \sum_{q \in \mathcal{P}_{\boldsymbol{\Omega}^{(2)}}} \operatorname{Res}_{z_{2}=q}\left[\psi_{i}^{(q)}\left(\mathbf{C}_{(\mathbf{1})}\right)_{i j} \varphi_{R, j}^{(2)}\right]
$$

where $\psi_{i}^{(q)}$ is the local solution of the differential equation

$$
\nabla_{\boldsymbol{\Omega}^{(2)}} \psi_{i}^{(q)}=d_{z_{2}} \psi_{i}^{(q)}+\psi_{j}^{(q)} \wedge \boldsymbol{\Omega}_{j i}^{(2)}=\varphi_{L, i}^{(2)},
$$

around each point $q \in \mathcal{P}_{\boldsymbol{\Omega}^{(2)}} \equiv\left\{\right.$ poles of $\boldsymbol{\Omega}^{(2)}$ (including $\infty$ ) .

As shown in eq. (3.18), the intersection number of 2 -forms $\left\langle\varphi_{L}^{(\mathbf{2})} \mid \varphi_{R}^{(\mathbf{2})}\right\rangle$ has been expressed in terms of quantities that are either intersection numbers of 1-forms or can be derived through them.

Example. Let us consider intersection numbers for integrals of the type in eq. (3.9), where:

$$
u(\mathbf{z})=\left(z_{1} z_{2}\left(1-z_{1}-z_{2}\right)\right)^{\gamma},
$$

which gives

$$
\hat{\omega}_{1}=\gamma\left(\frac{1}{z_{1}}-\frac{1}{1-z_{1}-z_{2}}\right), \quad \hat{\omega}_{2}=\gamma\left(\frac{1}{z_{2}}-\frac{1}{1-z_{1}-z_{2}}\right) .
$$

We will focus on the steps required for the computation of the self-intersection number of the 2-form $\langle 1| \equiv d z_{1} \wedge d z_{2}$ (simply given as 1 times the wedge product of the two elementary differentials), which, using the notation introduced above, can be written as

$$
\left\langle\varphi_{L}^{(\mathbf{2})} \mid \varphi_{R}^{(\mathbf{2})}\right\rangle \quad \text { with } \quad \hat{\varphi}_{L}^{(\mathbf{2})}=\hat{\varphi}_{R}^{(\mathbf{2})}=1 .
$$


Within the iterative approach, we consider first the integration in $z_{1}$ and define $\mathbf{1}=\{1\}$. Since $\hat{\omega}_{1}=0$ has one solution,

$$
\nu_{1}=1
$$

implying that the number of master 1 -forms in $z_{1}$ is just 1 . Therefore, we choose the inner basis for the left and right forms, denoted by $\left\langle e^{(\mathbf{1})}\right|$ and $\left|h^{(\mathbf{1})}\right\rangle$ respectively, as

$$
\hat{e}^{(\mathbf{1})}=\hat{h}^{(\mathbf{1})}=z_{1} .
$$

Given two arbitrary forms $\left\langle\varphi_{L}^{(\mathbf{2})}\right|$ and $\left|\varphi_{R}^{(2)}\right\rangle$, we decompose them as,

$$
\left\langle\varphi_{L}^{(\mathbf{2})}\left|=\left\langle e^{(\mathbf{1})}\left|\wedge\left\langle\varphi_{L}^{(2)}|, \quad| \varphi_{R}^{(\mathbf{2})}\right\rangle=\right| h^{(\mathbf{1})}\right\rangle \wedge\right| \varphi_{R}^{(2)}\right\rangle
$$

where $\left\langle\varphi_{L}^{(2)}\right|$ and $\left|\varphi_{R}^{(2)}\right\rangle$, are 1-forms in the variable $z_{2}$, and can be determined by projecting the 2 -forms on the bases of 1 -forms, using eqs. (3.14) and (3.15):

$$
\left\langle\varphi_{L}^{(2)}\left|=\left\langle\varphi_{L}^{(\mathbf{2})} \mid h^{(\mathbf{1})}\right\rangle \mathbf{C}_{(\mathbf{1})}^{-1}, \quad\right| \varphi_{R}^{(2)}\right\rangle=\mathbf{C}_{(\mathbf{1})}^{-1}\left\langle e^{(\mathbf{1})} \mid \varphi_{R}^{(\mathbf{2})}\right\rangle,
$$

with

$$
\mathbf{C}_{(\mathbf{1})}=\left\langle e^{(\mathbf{1})} \mid h^{(\mathbf{1})}\right\rangle
$$

Within the recursive approach, the evaluation of the required intersection numbers of 1 forms w.r.t. $z_{1}$ constitutes the first step, and they are given by,

$$
\begin{aligned}
\mathbf{C}_{(\mathbf{1})} & =\left\langle z_{1} \mid z_{1}\right\rangle=\frac{\gamma\left(z_{2}-1\right)^{4}}{8(2 \gamma-1)(2 \gamma+1)}, \\
\hat{\varphi}_{L}^{(2)} & =\left\langle 1 \mid z_{1}\right\rangle \mathbf{C}_{(\mathbf{1})}^{-1}=\frac{-2}{z_{2}-1}, \\
\hat{\varphi}_{R}^{(2)} & =\mathbf{C}_{(\mathbf{1})}^{-1}\left\langle z_{1} \mid 1\right\rangle=\frac{-2}{z_{2}-1} .
\end{aligned}
$$

Univariate intersection numbers in $z_{1}$, are also needed to compute the $(1 \times 1)$ connection matrix $\hat{\boldsymbol{\Omega}}^{(2)}$,

$$
\hat{\boldsymbol{\Omega}}^{(2)}=\left\langle\left(\partial_{z_{2}}+\hat{\omega}_{2}\right) z_{1} \mid z_{1}\right\rangle \mathbf{C}_{(\mathbf{1})}^{-1}=\frac{(3 \gamma+2) z_{2}-\gamma}{\left(z_{2}-1\right) z_{2}},
$$

which is needed for the next step. We observe that the set of the poles of $\hat{\boldsymbol{\Omega}}^{(2)}$ is,

$$
\mathcal{P}_{2}=\{0,1, \infty\}
$$

Next, we consider the differential equation:

$$
\left(\partial_{z_{2}}+\hat{\boldsymbol{\Omega}}^{(2)}\right) \psi^{(2)}=\hat{\varphi}_{L}^{(2)} .
$$

The full analytic solution of (3.33) is not required, but rather a power series around each $p \in \mathcal{P}_{2}$ is sufficient. Denoting by $y$ the local coordinate around the pole, the solutions of (3.33) to leading orders in $y$ read:

- Solution around $p=0\left(y=z_{2}\right)$ :

$$
\psi_{0}^{(2)}(y)=\frac{2 y}{\gamma+1}+\mathcal{O}\left(y^{2}\right) ;
$$


- Solution around $p=1\left(y=z_{2}-1\right)$ :

$$
\psi_{1}^{(2)}(y)=-\frac{1}{\gamma+1}+\mathcal{O}\left(y^{1}\right)
$$

- Solution around $p=\infty\left(y=1 / z_{2}\right)$ :

$$
\psi_{\infty}^{(2)}(y)=c_{0, \infty}+c_{1, \infty} y+c_{2, \infty} y^{2}+c_{3, \infty} y^{3}+c_{4, \infty} y^{4}+\mathcal{O}\left(y^{5}\right)
$$

with

$$
\begin{array}{rlrl}
c_{0, \infty} & =\frac{-2}{3 \gamma+2}, & c_{1, \infty}=\frac{-2 \gamma}{(3 \gamma+1)(3 \gamma+2)}, \\
c_{2, \infty}=\frac{-2(\gamma-1)}{3(3 \gamma+1)(3 \gamma+2)}, & c_{3, \infty}=\frac{-2(\gamma-2)(\gamma-1)}{3(3 \gamma-1)(3 \gamma+1)(3 \gamma+2)}, \\
c_{4, \infty}=\frac{-2(\gamma-3)(\gamma-2)(\gamma-1)}{3(3 \gamma-2)(3 \gamma-1)(3 \gamma+1)(3 \gamma+2)} . &
\end{array}
$$

Finally, we may evaluate the bi-variate intersection number as a sum of univariate residues, as given by eq. (3.18):

$$
\left\langle\varphi_{L}^{(\mathbf{2})} \mid \varphi_{R}^{(\mathbf{2})}\right\rangle=\sum_{p \in \mathcal{P}_{2}} \operatorname{Res}_{z_{2}=p}\left(\psi^{(2)} \mathbf{C}_{(\mathbf{1})} \varphi_{R}^{(2)}\right)
$$

yielding the final expression,

$$
\langle 1 \mid 1\rangle=\frac{\gamma^{2}}{3(3 \gamma-2)(3 \gamma-1)(3 \gamma+1)(3 \gamma+2)} .
$$

\subsection{Intersection numbers for $n$-forms}

Following the above discussion, we can generalize the intersection number of 2 -forms to the case of $n$-forms. In this case, we start by considering an integral with $n$ integration variables $\left(z_{1}, z_{2}, \ldots, z_{n}\right)$, written as

$$
\left.I\left(z_{1}, z_{2}, \ldots, z_{n}\right)=\int_{\mathcal{C}_{R}^{(\mathbf{n})}} \varphi_{L}^{(\mathbf{n})}\left(z_{1}, z_{2}, \ldots, z_{n}\right) u\left(z_{1}, z_{2}, \ldots, z_{n}\right)=\left\langle\varphi_{L}^{(\mathbf{n})}\right| \mathcal{C}_{R}^{(\mathbf{n})}\right]
$$

with the notation $\mathbf{n}=\{1, \ldots, n\}$. The $\varphi_{L}^{(\mathbf{n})}$ is an $n$-variable differential form on some space $X$. Similarly, one can define a dual form $\varphi_{R}^{(\mathbf{n})}$. We assume that the $n$-complex-dimensional space with coordinates $\left(z_{1}, \ldots, z_{n}\right)$ admits a fibration into a $(n-1)$-dimensional subspace parametrized by $\left(z_{1}, \ldots, z_{n-1}\right)$, denoted by $\mathbf{n}-\mathbf{1}$, which we call the inner space, and a one-dimensional subspace with $z_{n}$, which we refer to as the outer space. We have

$$
\omega=d \log u(\mathbf{z})=\sum_{i=1}^{n} \hat{\omega}_{i} d z_{i}
$$

and employing eq. (2.15), we can count the number of MIs on the inner space, which we define as $\nu_{\mathbf{n}-\mathbf{1}}$. The aim is to express intersection number for $n$-forms $\left\langle\varphi_{L}^{(\mathbf{n})} \mid \varphi_{R}^{(\mathbf{n})}\right\rangle$ in terms 
of intersection numbers for ( $n-1)$-forms on the inner space, which are assumed to be known at this stage, following the recursive nature of the algorithm. The choice of the variables (and their ordering) parametrizing the inner and outer spaces is arbitrary: as before, we use the generic notation $\mathbf{k} \equiv\left\{i_{1}, i_{2}, \ldots, i_{k}\right\}$ to denote the variables taking part in a specific computation.

Thus, the original $\mathbf{n}$-forms can be decomposed according to

$$
\begin{aligned}
\left\langle\varphi_{L}^{(\mathbf{n})}\right| & =\sum_{i=1}^{\nu_{\mathbf{n}-\mathbf{1}}}\left\langle e_{i}^{(\mathbf{n}-\mathbf{1})}\right| \wedge\left\langle\varphi_{L, i}^{(n)}\right|, \\
\left|\varphi_{R}^{(\mathbf{n})}\right\rangle & =\sum_{i=1}^{\nu_{\mathbf{n}-1}}\left|h_{i}^{(\mathbf{n}-\mathbf{1})}\right\rangle \wedge\left|\varphi_{R, i}^{(n)}\right\rangle,
\end{aligned}
$$

where $\nu_{\mathbf{n}-\mathbf{1}}$ is the number of master integrals on the inner space with arbitrary bases $\left\langle e_{i}^{(\mathbf{n}-1)}|,| h_{i}^{(\mathbf{n}-\mathbf{1})}\right\rangle$. In the above expressions $\left\langle\varphi_{L, i}^{(n)}\right|$ and $\left|\varphi_{R, i}^{(n)}\right\rangle$ are one-forms in the variable $z_{n}$, and they treated as coefficients of the basis expansion. They can be obtained by a projection similar to eq. (2.31), giving

$$
\begin{aligned}
\left\langle\varphi_{L, i}^{(n)}\right| & =\left\langle\varphi_{L}^{(\mathbf{n})} \mid h_{j}^{(\mathbf{n}-\mathbf{1})}\right\rangle\left(\mathbf{C}_{(\mathbf{n}-\mathbf{1})}^{-1}\right)_{j i}, \\
\left|\varphi_{R, i}^{(n)}\right\rangle & =\left(\mathbf{C}_{(\mathbf{n}-\mathbf{1})}^{-1}\right)_{i j}\left\langle e_{j}^{(\mathbf{n}-\mathbf{1})} \mid \varphi_{R}^{(\mathbf{n})}\right\rangle,
\end{aligned}
$$

with

$$
\left(\mathbf{C}_{(\mathbf{n}-\mathbf{1})}\right)_{i j}=\left\langle e_{i}^{(\mathbf{n}-\mathbf{1})} \mid h_{j}^{(\mathbf{n}-\mathbf{1})}\right\rangle .
$$

It is important to remark that, within the recursive approach, the intersection numbers of $(\mathbf{n}-\mathbf{1})$-forms (depending on $(\mathbf{n}-\mathbf{1})$ variables) are assumed to be known. The recursive formula for the intersection number reads [14]:

$$
\left\langle\varphi_{L}^{(\mathbf{n})} \mid \varphi_{R}^{(\mathbf{n})}\right\rangle=\sum_{p \in \mathcal{P}_{n}} \operatorname{Res}_{z_{n}=p}\left(\psi_{i}^{(n)}\left(\mathbf{C}_{(\mathbf{n}-\mathbf{1})}\right)_{i j} \varphi_{R, j}^{(n)}\right)
$$

where the functions $\psi_{i}^{(n)}$ are the solution of the system of differential equations

$$
\partial_{z_{n}} \psi_{i}^{(n)}+\psi_{j}^{(n)} \hat{\mathbf{\Omega}}_{j i}^{(n)}=\hat{\varphi}_{L, i}^{(n)},
$$

and $\hat{\varphi}_{L, i}$ are obtained through eq. (3.44). Here, $\hat{\boldsymbol{\Omega}}^{(n)}$ is a $\nu_{\mathbf{n}-\mathbf{1}} \times \nu_{\mathbf{n}-\mathbf{1}}$ matrix, whose entries are given by

$$
\hat{\mathbf{\Omega}}_{j i}^{(n)}=\left\langle\left(\partial_{z_{n}}+\hat{\omega}_{n}\right) e_{j}^{(\mathbf{n}-\mathbf{1})} \mid h_{k}^{(\mathbf{n}-\mathbf{1})}\right\rangle\left(\mathbf{C}_{(\mathbf{n}-\mathbf{1})}^{-1}\right)_{k i}
$$

and finally $\mathcal{P}_{n}$ is the set of poles of $\hat{\boldsymbol{\Omega}}^{(n)}$ defined as the union of the poles of its entries (including a possible pole at infinity).

We observe that the solution of eq. (3.48) around $z_{n}=p$ can be formally written in terms of a path-ordered matrix exponential

$$
\vec{\psi}^{(n)}\left(z_{n}\right)=\left(\mathcal{P} e^{-\int_{p}^{z_{n}} \boldsymbol{\Omega}^{(n)^{\mathrm{T}}}(w)}\right)\left(\int_{p}^{z_{n}} \mathcal{P} e^{\int_{p}^{y} \boldsymbol{\Omega}^{(n)^{\mathrm{T}}}(w)} \vec{\varphi}_{L}^{(n)}(y)\right)
$$


for a vector $\vec{\psi}^{(n)}$ with entries $\psi_{i}^{(n)}$. Nevertheless for its use in eq. (3.47), it is sufficient to know only a few leading orders of $\vec{\psi}^{(n)}$ around each $p \in \mathcal{P}_{n}$. Therefore, it is easier to find the solution of the system eq. (3.48) by a holomorphic Laurent series expansion, using an ansatz for each component $\psi_{i}^{(n)}$, see $[1,2]$. Such a solution exists if the matrix $\operatorname{Res}_{z_{n}=p} \boldsymbol{\Omega}^{(n)}$ does not have any non-negative integer eigenvalues, which we assume from now on (when this is not the case one can employ a regularization discussed in section 4.1). Moreover, the number of critical points of the determinant of the $\boldsymbol{\Omega}^{(n)}$ provides the dimension of that cohomology group, i.e. the number of the corresponding master forms [20].

The recursion terminates when $n=1$, in which case the inner space is trivial: $\nu_{\mathbf{0}}=\left\langle e_{1}^{(\mathbf{0})}|=| h_{1}^{(\mathbf{0})}\right\rangle=1$, and we impose the initial conditions

$$
\hat{\mathbf{\Omega}}_{11}^{(1)}=\hat{\omega}_{1}, \quad \mathbf{C}_{\mathbf{0}}=1, \quad \varphi_{L, 1}^{(1)}=\varphi_{L}^{(\mathbf{1})}, \quad \varphi_{R, 1}^{(1)}=\varphi_{R}^{(\mathbf{1})} .
$$

In this case eq. (3.47) reduces to a computation of an univariate intersection number $[1,2$, $4,6]$, discussed in section 3.3 .

Let us observe that the matrix $\boldsymbol{\Omega}^{(n)}$ is important to define the equivalence classes,

$$
\varphi_{L}^{(n)} \sim \varphi_{L}^{(n)}+\nabla_{\boldsymbol{\Omega}}^{(n)} \xi\left(z_{n}\right),
$$

where the covariant derivative, defined as $\nabla_{\boldsymbol{\Omega}}^{(n)} \equiv \mathbb{I} d_{z_{n}}+\boldsymbol{\Omega}^{(n)},\left(\mathbb{I}\right.$ is the $\left(\nu_{\mathbf{n}-\mathbf{1}} \times \nu_{\mathbf{n}-\mathbf{1}}\right)$ identity matrix in the $(\mathbf{n}-\mathbf{1})$ subspace) acts on any arbitrary function $\xi\left(z_{n}\right)$ - see appendix A for a formal derivation.

\subsubsection{Explicit formula}

Let us notice also that combining eqs. (3.47) and (3.45) gives

$$
\left\langle\varphi_{L}^{(\mathbf{n})} \mid \varphi_{R}^{(\mathbf{n})}\right\rangle=\sum_{p \in \mathcal{P}_{n}} \operatorname{Res}_{z_{n}=p}\left(\psi_{i}^{(n)}\left\langle e_{i}^{(\mathbf{n}-\mathbf{1})} \mid \varphi_{R}^{(\mathbf{n})}\right\rangle\right)
$$

which is suitable for practical calculation purposes. Using the above identity recursively, the intersection number can be expressed as,

$$
\left\langle\varphi_{L}^{(\mathbf{n})} \mid \varphi_{R}^{(\mathbf{n})}\right\rangle=\sum_{p_{n} \in \mathcal{P}_{n}} \cdots \sum_{p_{1} \in \mathcal{P}_{1}} \operatorname{Res}_{z_{n}=p_{n}} \cdots \operatorname{Res}_{z_{1}=p_{1}}\left(\psi_{i_{\mathbf{n}-\mathbf{1}}}^{(n)} \psi_{i_{\mathbf{n}-1} i_{\mathbf{n}-\mathbf{2}}}^{(n-1)} \cdots \psi_{i_{\mathbf{2}} i_{\mathbf{1}}}^{(2)} \psi_{i_{1} 1}^{(1)} \varphi_{R}^{(\mathbf{n})}\right),
$$

where the ranges of the summations are $i_{\mathbf{m}}=1, \ldots, \nu_{\mathbf{m}}$ and where the $\psi_{i_{\mathbf{m}} i_{\mathbf{m}-\mathbf{1}}}^{(m)}$ are the solutions of

$$
\partial_{z_{m}} \psi_{i_{\mathbf{m}} i_{\mathbf{m}-\mathbf{1}}}^{(m)}+\psi_{i_{\mathbf{m}} j_{\mathbf{m}-\mathbf{1}}}^{(m)} \hat{\mathbf{\Omega}}_{j_{\mathbf{m}-\mathbf{1}} i_{\mathbf{m}-\mathbf{1}}}^{(m)}=\hat{e}_{i_{\mathbf{m}} i_{\mathbf{m}-\mathbf{1}}}^{(m)}
$$

for all $i_{\mathbf{m}}$ with $\left\langle e_{i_{\mathbf{m}} i_{\mathbf{m}-\mathbf{1}}}^{(m)}\right|=\hat{e}_{i_{\mathbf{m}} i_{\mathbf{m}-\mathbf{1}}}^{(m)} d z_{m}$ coming from the projection

$$
\left\langle e_{i_{\mathbf{m}}}^{(\mathbf{m})}\right|=\left\langle e_{i_{\mathbf{m}-\mathbf{1}}}^{(\mathbf{m}-\mathbf{1})}\right| \wedge\left\langle e_{i_{\mathbf{m}} i_{\mathbf{m}-\mathbf{1}}}^{(m)}\right|,
$$

which may be computed initially, since the bases of all inner spaces are arbitrarily chosen. The matrices $\hat{\boldsymbol{\Omega}}^{(m)}$ needed in eq. (3.55) are computed analogously to eq. (3.49). Notice that all $\psi^{(m)}$ entering eq. (3.54) need to be computed only once for a given family of integrals. 


\subsubsection{Dual formula}

Let us discuss an alternative recursive formula for intersection numbers, which uses the dual connection matrix $\widetilde{\boldsymbol{\Omega}}^{(n)}$ instead of $\boldsymbol{\Omega}^{(n)}$. This amounts to repeating the same steps presented in the former section, but using the decomposition of the differential dual-forms given in eq. (3.43) (instead of eq. (3.42)),

$$
\left\langle\varphi_{L}^{(\mathbf{n})} \mid \varphi_{R}^{(\mathbf{n})}\right\rangle=-\sum_{i, j=1}^{\nu_{1}} \sum_{q \in \mathcal{P}_{n}} \operatorname{Res}_{z_{n}=q}\left[\varphi_{L, i}^{(n)}\left(\mathbf{C}_{(\mathbf{n}-\mathbf{1})}\right)_{i j} \psi_{j}^{(q)}\right],
$$

where,

$$
\widetilde{\mathbf{\Omega}}_{i j}^{(n)}=-\left(\mathbf{C}_{(\mathbf{n}-\mathbf{1})}^{-1}\right)_{i k}\left\langle e_{k}^{(\mathbf{n}-\mathbf{1})} \mid\left(d_{z_{n}}-\omega_{n} \wedge\right) h_{j}^{(\mathbf{n}-\mathbf{1})}\right\rangle,
$$

$\mathcal{P}_{n}$ is the set of its poles (including the pole at $\infty$ ), and $\psi_{j}^{(q)}$ is the solution of

$$
\nabla_{\widetilde{\mathbf{\Omega}}^{(n)}} \psi_{j}^{(q)}=d_{z_{n}} \psi_{j}^{(q)}-\widetilde{\boldsymbol{\Omega}}_{j i}^{(n)} \wedge \psi_{i}^{(q)}=\varphi_{R, j}^{(n)} .
$$

The dual formula provides a useful consistency check for the computation of intersection numbers, and it can be combined with the original formula to devise efficient evaluation algorithms which can better exploit the pole structure of $\varphi_{L}, \varphi_{R}$, and of the chosen bases $e_{i}$ and $h_{i}$, in order to minimize the computational load.

\subsection{Simplifying the computation of intersection numbers}

The recursive algorithm for the computation of the multivariate intersection numbers presented in section 3 is applicable for any rational form. However, at each step of the recursive algorithm, the coefficients $\hat{\varphi}_{L, R}^{(n)}$ in eqs. (3.42), (3.43) are defined modulo the equivalence relations

$$
\begin{aligned}
& \hat{\varphi}_{L, i}^{(n)} \sim \hat{\varphi}_{L, i}^{(n)}=\hat{\varphi}_{L, i}^{(n)}+\left(\partial_{z_{n}} \xi_{L, i}+\xi_{L, j} \hat{\boldsymbol{\Omega}}_{j i}^{(n)}\right), \\
& \hat{\varphi}_{R, i}^{(n)} \sim \hat{\varphi}_{R, i}^{(n)}=\hat{\varphi}_{R, i}^{(n)}+\left(\partial_{z_{n}} \xi_{R, i}-\hat{\widetilde{\Omega}}_{i j}^{(n)} \xi_{R, j}\right) .
\end{aligned}
$$

Thus, under the assumption that the connection matrices $\boldsymbol{\Omega}^{(n)}$ and $\widetilde{\boldsymbol{\Omega}}^{(n)}$ contain only simple poles, its possible to replace the coefficients $\hat{\varphi}_{L, R}^{(n)}$ containing higher-degree poles, with a suitably chosen $\hat{\varphi}_{L, R}^{(n)}$ belonging to the same equivalence class, but containing simple poles only. One may exploit this fact to compute intersection numbers in one variable as a univariate global residue, without introducing any algebraic extensions as observed in [20].

\section{Feynman integral decomposition}

As proposed in refs. $[1-3,17,20]$, the use of multivariate intersection numbers yields a direct decomposition of a given Feynman integral $I$ in terms of an a priori chosen set of MIs $J_{i}$, with $i=1, \ldots, \nu$. 
The decomposition given by eq. (2.27) is on the form

$$
I=\sum_{i=1}^{\nu} c_{i} J_{i}
$$

where the determination of the coefficients $c_{i}$ is the goal of this section. We identify three possible strategies which can be adopted in order to achieve this task. They all employ the master projection formula from eq. (2.31), which is applied to differential forms constructed differently in the three cases. We name them the straight decomposition, the bottom-up decomposition, and the top-down decomposition.

All the approaches have the first step in common: finding the number of MIs which appear in the decomposition and choosing them accordingly.

We introduce the following definitions:

- $\Sigma$ denotes the set of integers used to label the full set of denominators;

- $\sigma$ denotes a set of integers that label a subset of denominators, $\sigma \subseteq \Sigma$;

- sector is the set of integrals for which only the subset of propagators specified by $\sigma$ appear in the denominator (thus, a sector is unambiguously identified by $\sigma$ ).

There is a one-to-one correspondence between sectors and (generalized unitarity) cuts. On the level of the function $u$, this correspondence is manifested by setting all $z_{j}$ 's belonging to $\sigma$ to zero in the original $u(\mathbf{z})$,

$$
u_{\sigma}=\left.u(\mathbf{z})\right|_{z_{j \in \sigma} \rightarrow 0},
$$

where we work in Baikov representation. Given $u_{\sigma}$, the number of MIs in the corresponding sector, $\nu_{\sigma}$, can be determined through the criteria given in section 2.2. The total number of MIs (without taking into account any symmetry relations) is then given by

$$
\nu=\sum_{\sigma} \nu_{\sigma}
$$

where the sum is over all sectors. Finally we can choose the forms $\left\langle e_{i}\right|$ associated to the (arbitrarily chosen) MIs $J_{i}$, through the identification

$$
\left.J_{i}=\left\langle e_{i}\right| \mathcal{C}\right]
$$

\subsection{Straight decomposition}

We consider the following decomposition

$$
\left.\left.I=\int_{\mathcal{C}} u \varphi=\langle\varphi| \mathcal{C}\right]=\sum_{i=1}^{\nu} c_{i}\left\langle e_{i}\right| \mathcal{C}\right]=\sum_{i=1}^{\nu} c_{i} \int_{\mathcal{C}} u e_{i}=\sum_{i=1}^{\nu} c_{i} J_{i}
$$

with

$$
c_{i}=\sum_{j=1}^{\nu}\left\langle\varphi \mid h_{j}\right\rangle\left(\mathbf{C}^{-1}\right)_{j i}, \quad \mathbf{C}_{i j}=\left\langle e_{i} \mid h_{j}\right\rangle .
$$


Here $\hat{\varphi}$ and $\hat{e}_{i}$ correspond simply to the integrands of the integral $I$ to decompose and of the chosen master integrals, $J_{i}$, respectively. In order to evaluate the intersection numbers, all the poles present in the differential forms must be regulated in $u$. If this assumption is violated, we can introduce a regulated $u$, denoted by $u_{\rho}$, which contains a monomial $z_{k}^{\rho_{k}}$ for each (non-regulated) pole present in the differential forms, that is

$$
u_{\rho}(\mathbf{z})=\left(\prod_{k \in \Sigma} z_{k}^{\rho_{k}}\right) u(\mathbf{z})
$$

and correspondingly

$$
\omega_{\rho}(\mathbf{z})=d \log u_{\rho}(\mathbf{z})=d \log u(\mathbf{z})+\sum_{k \in \Sigma} \rho_{k} \frac{d z_{k}}{z_{k}}=\omega(\mathbf{z})+\sum_{k \in \Sigma} \rho_{k} \frac{d z_{k}}{z_{k}},
$$

where we emphasized the action of regulators. By analogy, we also introduce a regularized version of $\hat{\boldsymbol{\Omega}}^{(n)}$, whenever $\operatorname{Res}_{z_{n}=p} \hat{\mathbf{\Omega}}^{(n)}$ has any non-negative integer eigenvalue. The regularized $\hat{\boldsymbol{\Omega}}^{(n)}$ reads:

$$
\hat{\mathbf{\Omega}}_{\Lambda}^{(n)}=\hat{\mathbf{\Omega}}^{(n)}+\frac{\Lambda}{z_{n}-p} \mathbb{I} .
$$

Thus, we obtain a new system of differential equations, analogous to eq. (3.48), which is, in this case, controlled by $\hat{\mathbf{\Omega}}_{\Lambda}^{(n)}$. We assume that the solution of the latter around a pole $p$, denoted by $\psi_{\Lambda, p}^{(n)}$, reproduces in the limit $\Lambda \rightarrow 0$, a solution for the original system (around the pole $p$ ).

The intersection numbers are computed through $\omega_{\rho}$, and lead to a set of coefficients, denoted by $c_{\rho, i}$, which depend on the set of regulators, collectively indicated by $\rho$. The coefficients $c_{i}$, which appear in the original decomposition eq. (4.5), are recovered in the limit $\rho \rightarrow 0,^{3}$

$$
c_{i}=\lim _{\rho \rightarrow 0} c_{\rho, i}=\lim _{\rho \rightarrow 0} \sum_{j=1}^{\nu}\left\langle\varphi \mid h_{j}\right\rangle_{\rho}\left(\mathbf{C}_{\rho}^{-1}\right)_{j i}, \quad\left(\mathbf{C}_{\rho}\right)_{i j}=\left\langle e_{i} \mid h_{j}\right\rangle_{\rho} .
$$

This approach requires the evaluation of intersection numbers, for which all the integration variables are present simultaneously.

For ease of notation, whenever the regulated $u$ is introduced, in the following we will omit the subscript $\rho$ from the individual intersection numbers $\left\langle\varphi \mid h_{j}\right\rangle_{\rho}$ and $\left\langle e_{i} \mid h_{j}\right\rangle_{\rho}$.

\subsection{Bottom-up decomposition}

In this approach, proposed in [3], the decomposition is applied to the spanning set of cuts, defined as the minimal set of cuts such that each MIs appears at least once [3, 69] (a cut

\footnotetext{
${ }^{3}$ Strictly speaking, we take it as an assumption that the limit $\rho \rightarrow 0$ is smooth, which turns out to be true in all practical examples we studied. This might seem reasonable given that the regularization used in (4.7) is a version of analytic regularization for Feynman integrals [56] which cannot have poles in $\rho_{k}$ as long as dimensional regularization is also employed. However, there might exist situations where a MI $J_{i}$ has a zero in $\rho$ compensated by a pole in $\rho$ of $c_{i}$, leading to a finite result: in this case, the product $c_{i} J_{i}$ has a smooth limit, but not each term individually.
} 
behave like a high-pass filter, therefore MIs whose denominators do not contain all the cutdenominators will not contribute to the decomposition on that cut). We denote a given spanning cut (i.e. an element in the spanning set of cuts) by $\tau$; moreover $\mathcal{S}_{\tau}$ is the set of sectors which survive on that spanning cut

$$
\mathcal{S}_{\tau}=\{\sigma \mid \sigma \supseteq \tau\} .
$$

Finally, the number of MIs which survive on the spanning cut $\tau$, denoted by $\nu_{\mathcal{S}_{\tau}}$ is

$$
\nu_{\mathcal{S}_{\tau}}=\sum_{\sigma \in \mathcal{S}_{\tau}} \nu_{\sigma}
$$

On the spanning cut $\tau$, we define

$$
u_{\tau}=\left.u(\mathbf{z})\right|_{z_{j \in \tau} \rightarrow 0}
$$

and we consider the following decomposition

$$
\begin{aligned}
\left.I_{\tau}=\int_{\mathcal{C}_{\tau}} u_{\tau} \varphi_{\tau}=\left\langle\varphi_{\tau}\right| \mathcal{C}_{\tau}\right] & \left.=\sum_{i=1}^{\nu_{\mathcal{S}_{\tau}}} c_{i}\left\langle e_{i, \tau}\right| \mathcal{C}_{\tau}\right] \\
& =\sum_{i=1}^{\nu_{\mathcal{S}_{\tau}}} c_{i} \int_{\mathcal{C}_{\tau}} u_{\tau} e_{i, \tau}=\sum_{i=1}^{\nu_{\mathcal{S}_{\tau}}} c_{i} J_{i, \tau}
\end{aligned}
$$

with

$$
c_{i}=\sum_{j=1}^{\nu_{\mathcal{S}_{\tau}}}\left\langle\varphi_{\tau} \mid h_{j, \tau}\right\rangle\left(\mathbf{C}^{-1}\right)_{j i}, \quad \mathbf{C}_{i j}=\left\langle e_{i, \tau} \mid h_{j, \tau}\right\rangle .
$$

As expected, $\hat{\varphi}_{\tau}$ and $\hat{e}_{i, \tau}$ are inferred from the cut-integrals. As in any unitarity-based approach [70-72], the coefficients $c_{i}$ determined from a cut decomposition are identical to those appearing in the original decomposition - the coefficients are invariant under cuts. Therefore, the complete decomposition for the (uncut) integral $I$ can be obtained by combining the coefficients determined from the individual spanning cuts.

As described in subsection 4.1, all the poles present in the differential forms must be regulated in $u_{\tau}$. If this is not the case, we can introduce the regularized $u_{\tau}$, denoted by $u_{\rho, \tau}$

$$
u_{\rho, \tau}=\left(\prod_{k \in \Sigma \backslash \tau} z_{k}^{\rho_{k}}\right) u_{\tau}
$$

which leads to

$$
\omega_{\rho, \tau}=d \log u_{\rho, \tau}=d \log u(\mathbf{z})+\sum_{k \in \Sigma \backslash \tau} \rho_{k} \frac{d z_{k}}{z_{k}}=\omega(\mathbf{z})+\sum_{k \in \Sigma \backslash \tau} \rho_{k} \frac{d z_{k}}{z_{k}},
$$

used in the evaluation of the intersection number. We also use a regularized version of $\hat{\boldsymbol{\Omega}}^{(n)}$, whenever $\operatorname{Res}_{z_{n}=p} \hat{\boldsymbol{\Omega}}^{(n)}$ has any non-negative integer eigenvalue, as explained above. Now, the coefficients of the decomposition, $c_{\rho, i}$ depend on the set of regulators $\rho$. The coefficients of the original decomposition (4.14) are recovered in the $\rho \rightarrow 0$ limit:

$$
c_{i}=\lim _{\rho \rightarrow 0} c_{\rho, i}=\lim _{\rho \rightarrow 0} \sum_{j=1}^{\nu_{\mathcal{S}_{\tau}}}\left\langle\varphi_{\tau} \mid h_{j, \tau}\right\rangle_{\rho}\left(\mathbf{C}_{\rho}^{-1}\right)_{j i}, \quad\left(\mathbf{C}_{\rho}\right)_{i j}=\left\langle e_{i, \tau} \mid h_{j, \tau}\right\rangle_{\rho} .
$$


This procedure requires the evaluation of the intersection numbers only for the uncut variables, therefore it can be significantly less demanding than the previous case.

As before, whenever the regulated $u$ is introduced, we will omit the subscript $\rho$ from the individual intersection numbers.

\subsection{Top-down decomposition}

This approach is new and combines the advantages of the decomposition by intersection numbers with the top-down subtraction algorithm traditionally used in methods of integrand decomposition [33-40] In particular, as for the integrand decomposition, one can determine the coefficients of the MIs systematically, beginning from the ones with the highest number of internal lines (the top sector) and moving downward, ending with the sector with a minimal number of lines equal to the number of the loops (built from product of tadpoles). At any step, the determination of the coefficients of a given MI, say $J_{i}$, is obtained on the corresponding cut, after subtracting off the known contributions coming from higher sectors, as the latter are written as a linear combination of the MIs with a higher number of internal lines (whose graph contain the one corresponding to $J_{i}$ as subdiagram), coming from the earlier steps of the decomposition. In particular, let us reconsider the complete decomposition,

$$
\left.\left.I=\int_{\mathcal{C}} u \varphi=\langle\varphi| \mathcal{C}\right]=\sum_{i=1}^{\nu} c_{i}\left\langle e_{i}\right| \mathcal{C}\right]=\sum_{i=1}^{\nu} c_{i} \int_{\mathcal{C}} u e_{i}=\sum_{i=1}^{\nu} c_{i} J_{i}
$$

and assume that, within the top-down approach, after at most $n$-steps, the coefficients $c_{i}$, with $i=1, \ldots, n$ have been determined, and can be considered as known. We can write,

$$
I-\sum_{i=1}^{n} c_{i} J_{i}=\sum_{i=n+1}^{\nu} c_{i} J_{i},
$$

which, in terms of pairings, reads,

$$
\left.\left.\left\langle\phi_{n}\right| \mathcal{C}\right]=\sum_{i=1}^{n} c_{i}\left\langle e_{i}\right| \mathcal{C}\right],
$$

where $\left\langle\phi_{n}\right|$, defined as,

$$
\left\langle\phi_{n}\right| \equiv\langle\varphi|-\sum_{i=1}^{n} c_{i}\left\langle e_{i}\right|
$$

is a known differential form. By applying a cut $\tau$, namely $z_{j}=0, \forall j \in \tau$, we can then determine the coefficients $c_{i}$ for $i=n+1, \ldots n+\nu_{\tau}$, where $\nu_{\tau}$ is the number of those MIs that have as denominators exclusively (all and only) the cut ones, namely $z_{j}$, with $j \in \tau$. In fact, on the cut $\tau$, we can define

$$
u_{\tau}=\left.u(\mathbf{z})\right|_{z_{j \in \tau} \rightarrow 0}
$$

and

$$
\omega_{\tau}=d \log u_{\tau}
$$


and the decomposition simplifies and becomes,

$$
\begin{aligned}
\left.I_{\tau}=\int_{\mathcal{C}_{\tau}} u_{\tau} \phi_{n, \tau}=\left\langle\phi_{n, \tau}\right| \mathcal{C}_{\tau}\right] & \left.=\sum_{i=n+1}^{n+\nu_{\tau}} c_{i}\left\langle e_{i, \tau}\right| \mathcal{C}_{\tau}\right] \\
& =\sum_{i=n+1}^{n+\nu_{\tau}} c_{i} \int_{\mathcal{C}_{\tau}} u_{\tau} e_{i, \tau}=\sum_{i=n+1}^{n+\nu_{\tau}} c_{i} J_{i, \tau}
\end{aligned}
$$

with

$$
c_{n+i}=\sum_{j=1}^{\nu_{\tau}}\left\langle\phi_{n, \tau} \mid h_{n+j, \tau}\right\rangle\left(\mathbf{C}^{-1}\right)_{j i}, \quad \mathbf{C}_{i j}=\left\langle e_{n+i, \tau} \mid h_{n+j, \tau}\right\rangle .
$$

Two important observations are in order. First, we notice that the subtraction in eq. (4.20), is similar in spirit to the subtraction performed in an integrand decomposition, although the known coefficients depend also on $d$, and not only on the kinematical variables. Second, after the subtraction of the known terms, the differential form $\phi_{n, \tau}$ may contain spurious poles, which are not regulated by $u_{\tau}$. By exploiting the equivalence class properties, we build an equivalent form, $\phi_{n, \tau}^{\prime} \sim \phi_{n, \tau}$, which is free of them,

$$
\phi_{n, \tau}^{\prime} \equiv \phi_{n, \tau}+\nabla_{\omega_{\tau}} \xi_{L, \tau}
$$

for a suitable choice of $\xi_{L, \tau}$. Thus, in this approach, the regulators are not introduced. At this point the determination of the coefficients via intersection numbers can proceed iteratively, top-down, until all sectors have had their $c_{i}$ coefficients determined.

We would like to observe that the top-down decomposition algorithm offers the advantage of a unitarity-based integrand-decomposition in terms of a minimal bases of MIs.

Let us finally remark that the exploitation of relations within equivalence class of differential forms for eliminating the contributions of poles that do not appear as being regulated is a novel idea which we plan to elaborate on in the future: this approach might be interestingly combined with the more recent mathematical idea of relative twisted homology and cohomology groups [58], to be used for computing intersection numbers without regulators, as well as, more generally, to investigate the finiteness of scattering amplitudes around explicit dimensions.

\section{Examples}

In this section we illustrate the previously-discussed decomposition algorithms on a few examples.

\subsection{One-loop massless box}

As the first example we will discuss the one-loop massless box as shown in figure 1. This diagram was discussed in the context of intersection theory already in ref. [3], but we will here add further details, and go through the reduction with each of the three methods presented in section 4 .

The kinematics is such that

$$
\begin{array}{ll}
D_{1}=k^{2}, & D_{2}=\left(k+p_{1}\right)^{2}, \\
D_{3}=\left(k+p_{1}+p_{2}\right)^{2}, & D_{4}=\left(k+p_{1}+p_{2}+p_{3}\right)^{2},
\end{array}
$$

with $p_{i}^{2}=0,\left(p_{1}+p_{2}\right)^{2}=s,\left(p_{2}+p_{3}\right)^{2}=t,\left(p_{1}+p_{3}\right)^{2}=-s-t$. 


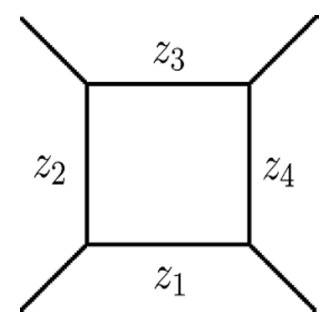

Figure 1. Massless box.

Performing the Baikov parametrization yields

$$
u=\mathcal{B}^{(d-5) / 2}
$$

with

$$
\begin{aligned}
\mathcal{B}= & 2 s t\left(s\left(z_{2}+z_{4}\right)+t\left(z_{1}+z_{3}\right)-z_{1} z_{2}-z_{2} z_{3}-z_{3} z_{4}-z_{4} z_{1}+2 z_{1} z_{3}+2 z_{2} z_{4}\right) \\
& -s^{2} t^{2}-t^{2}\left(z_{1}-z_{3}\right)^{2}-s^{2}\left(z_{2}-z_{4}\right)^{2}
\end{aligned}
$$

and performing the sector-by-sector analysis described in the beginning of section 4 yields $\nu_{\sigma}=1$ for the sectors

$$
\sigma \in\{\{1,2,3,4\},\{1,3\},\{2,4\}\}
$$

and $\nu_{\sigma}=0$ for the remaining sectors, corresponding to the well-known set of master integrals: the box and the $s$ - and the $t$-channel bubble:

$$
J_{1}=\square, \quad J_{2}=\searrow, \quad J_{3}=\square .
$$

The corresponding differential forms read

$$
\hat{e}_{1}=\frac{1}{z_{1} z_{2} z_{3} z_{4}}, \quad \hat{e}_{2}=\frac{1}{z_{1} z_{3}}, \quad \hat{e}_{3}=\frac{1}{z_{2} z_{4}} .
$$

In the following we will decompose the example

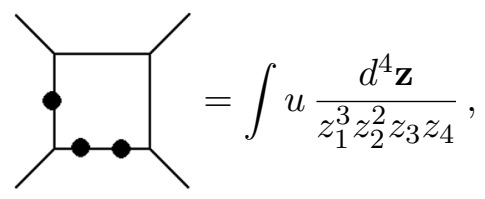

which can be expressed in terms of the chosen master integrals as

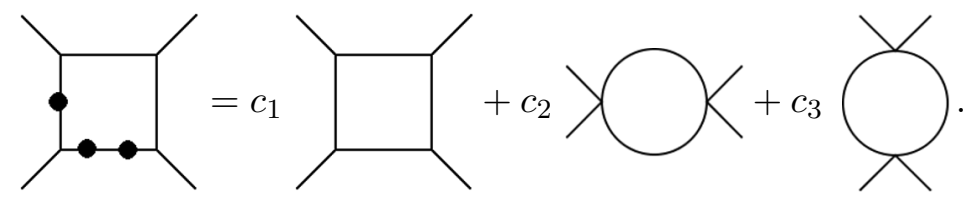

We will determine these coefficients with the three methods presented in section 4 . 


\subsubsection{Straight decomposition}

As prescribed in section 4.1 we may construct the regulated $u$ as

$$
u_{\rho}=u \times z_{1}^{\rho} z_{2}^{\rho} z_{3}^{\rho} z_{4}^{\rho},
$$

where in this case we pick the regulators to be all equal. From this definition we may construct the corresponding $\omega$ as

$$
\omega_{\rho}=\sum_{i=1}^{4} \hat{\omega}_{i} \mathrm{~d} z_{i} \quad \text { with } \quad \hat{\omega}_{i}=\partial_{z_{i}} \log u_{\rho} .
$$

Choosing the variable ordering to be, from the innermost to the outermost, $z_{4}, z_{3}, z_{2}, z_{1}$, we can compute the dimensions of the twisted cohomology groups corresponding to the individual layers of the fibration. The result is

$$
\nu_{\{4321\}}=3, \quad \nu_{\{432\}}=4, \quad \nu_{\{43\}}=3, \quad \nu_{\{4\}}=2 .
$$

Corresponding to the order of variables given above, we pick the basis for each level to be

$$
\begin{aligned}
\hat{e}^{(4321)}=\hat{e} & =\left\{\frac{1}{z_{1} z_{2} z_{3} z_{4}}, \frac{1}{z_{1} z_{3}}, \frac{1}{z_{2} z_{4}}\right\}, & \hat{e}^{(432)} & =\left\{\frac{1}{z_{2}}, \frac{1}{z_{3}}, \frac{1}{z_{2} z_{3}}, \frac{1}{z_{2} z_{3} z_{4}}\right\}, \\
\hat{e}^{(43)} & =\left\{\frac{1}{z_{4}}, \frac{1}{z_{3}}, \frac{1}{z_{3} z_{4}}\right\}, & \hat{e}^{(4)} & =\left\{\frac{1}{z_{4}}, 1\right\} .
\end{aligned}
$$

We choose the dual bases to be $\hat{h}_{i}=\hat{e}_{i}$. In the following, we will decompose

$$
\hat{\varphi}=\frac{1}{z_{1}^{3} z_{2}^{2} z_{3} z_{4}} .
$$

The required intersection numbers are

$$
\mathbf{C}_{i j}=\left\langle e_{i} \mid h_{j}\right\rangle, \quad 1 \leq i, j \leq 3,
$$

and

$$
\left\langle\varphi \mid h_{k}\right\rangle, \quad 1 \leq k \leq 3
$$

The individual intersection numbers, up to the leading order in $\rho$, are presented in appendix C.1.

Combining the intersection numbers as dictated by eq. (4.10), we obtain, after taking the limit $\rho \rightarrow 0$, the coefficients

$$
\begin{array}{ll}
c_{1} & =\frac{-(d-7)(d-6)(d-5)}{2 s^{2} t}, \\
c_{3} & =\frac{2(d-7)(d-5)(d-3)(2 s+(d-8) t)}{(d-8) s^{2} t^{4}}
\end{array}
$$

These results are in agreement with the values obtained with FIRE [73]. 


\subsubsection{Bottom-up decomposition}

The first step of a bottom-up decomposition is to identify a spanning set of cuts $\tau$. That set is easily seen to be the cuts corresponding the two bubbles

$$
\tau \in\{\{1,3\},\{2,4\}\} .
$$

Cut $\boldsymbol{\tau}=\{\mathbf{1}, \mathbf{3}\}$. Let us first consider the $\tau=\{1,3\}$ cut.

On this cut, the decomposition reads:

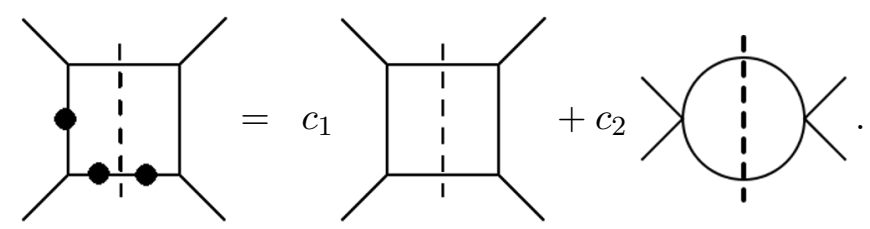

We have

$$
u_{\rho, \tau}=z_{2}^{\rho} z_{4}^{\rho} \mathcal{B}_{\tau}^{(d-5) / 2}(-s)^{(d-5) / 2}
$$

where

$$
\mathcal{B}_{\tau}=\left(s t^{2}+s\left(z_{2}-z_{4}\right)^{2}-2 t\left(s\left(z_{2}+z_{4}\right)+2 z_{2} z_{4}\right)\right),
$$

and $\omega_{\rho, \tau}=\hat{\omega}_{2} \mathrm{~d} z_{2}+\hat{\omega}_{4} \mathrm{~d} z_{4}$ with

$$
\hat{\omega}_{2}=\partial_{z_{2}} \log u_{\rho, \tau}, \quad \hat{\omega}_{4}=\partial_{z_{4}} \log u_{\rho, \tau} .
$$

The variable ordering, from the innermost to the outermost, is chosen as $z_{2}, z_{4}$. The dimensions of the cohomology groups read:

$$
\nu_{\{24\}}=2, \quad \nu_{\{2\}}=2 .
$$

The basis elements, on the cut, are:

$$
\hat{e}_{\tau}^{(24)}=\hat{e}_{\tau}=\left\{\frac{1}{z_{2} z_{4}}, 1\right\}, \quad \hat{e}_{\tau}^{(2)}=\left\{1, \frac{1}{z_{2}}\right\} .
$$

The dual basis elements are chosen as $\hat{h}_{i, \tau}=\hat{e}_{i, \tau}$.

We will show the decomposition, on the cut, of:

$$
\hat{\varphi}_{\tau}=\left.\frac{\frac{1}{2} \partial_{z_{1}}^{2} u}{u z_{2}^{2} z_{4}}\right|_{z_{1}, z_{3}=0}=\frac{(d-5) t^{2}\left((d-6) s\left(z_{2}+z_{4}-t\right)^{2}-4(s+t) z_{2} z_{4}\right)}{2 s z_{2}^{2} z_{4} \mathcal{B}_{\tau}^{2}} .
$$

This requires the intersection numbers

$$
\mathbf{C}_{i j}=\left\langle e_{i, \tau} \mid h_{j, \tau}\right\rangle, \quad 1 \leq i, j \leq 2,
$$

and

$$
\left\langle\varphi_{\tau} \mid h_{k, \tau}\right\rangle, \quad 1 \leq k \leq 2 .
$$

Expressions for the individual intersection numbers are presented in appendix C.1. Combining them as prescribed by eq. (4.18), and considering the limit $\rho \rightarrow 0$, we obtain the coefficients $c_{1}$ and $c_{2}$ in agreement with eq. (5.17). 
Cut $\tau=\{2,4\}$. Performing instead the decomposition on the second of the spanning cuts, $\tau=\{2,4\}$ will allow us to reconstruct $c_{1}$ and $c_{3}$ in eq. (5.17), which means that in total all of the master integral coefficients $c_{i}$ have been extracted.

\subsubsection{Top-down decomposition}

The first step in the top-down decomposition is the extraction of the box-coefficient.

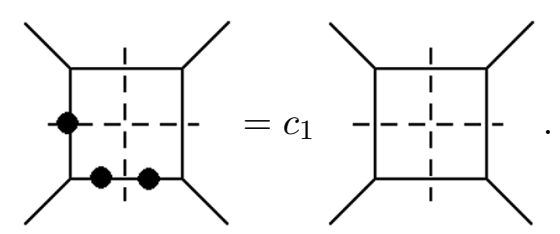

The coefficient $c_{1}$ can be computed as $\varphi / e_{1}$ on the maximal cut:

$$
c_{1}=\left.\frac{\frac{1}{2} \partial_{z_{1}}^{2} \partial_{z_{2}} u}{u}\right|_{z_{i} \rightarrow 0}=\frac{-(d-7)(d-6)(d-5)}{2 s^{2} t},
$$

in agreement with eqs. (5.17).

We then consider the $s$-channel bubble corresponding to the cut $\tau=\{1,3\}$.
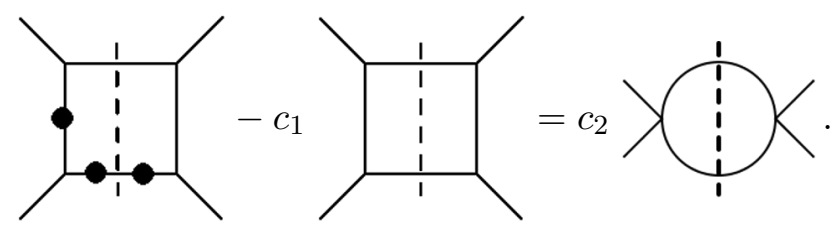

Here we have

$$
u_{\tau}=\mathcal{B}_{\tau}^{(d-5) / 2}(-s)^{(d-5) / 2} \quad \text { with } \quad \mathcal{B}_{\tau}=\left(s t^{2}+s\left(z_{2}-z_{4}\right)^{2}-2 t\left(s\left(z_{2}+z_{4}\right)+2 z_{2} z_{4}\right)\right),
$$

and

$$
\hat{\varphi}=\left.\frac{\frac{1}{2} \partial_{z_{1}}^{2} u}{u z_{2}^{2} z_{4}}\right|_{z_{1}, z_{3}=0}=\frac{(d-5) t^{2}\left((d-6) s\left(z_{2}+z_{4}-t\right)^{2}-4(s+t) z_{2} z_{4}\right)}{2 s z_{2}^{2} z_{4} \mathcal{B}_{\tau}^{2}} .
$$

We also get

$$
\omega=\frac{-(d-5)\left(\left(t\left(z_{4}-z_{2}\right)+s\left(t+2 z_{4}\right)\right) \mathrm{d} z_{2}+\left(s\left(t+2 z_{2}\right)+t\left(z_{2}-z_{4}\right)\right) \mathrm{d} z_{4}\right)}{\mathcal{B}_{\tau}}
$$

from which we can extract $\nu_{\tau}=1$ corresponding to the $s$-channel bubble.

We know that

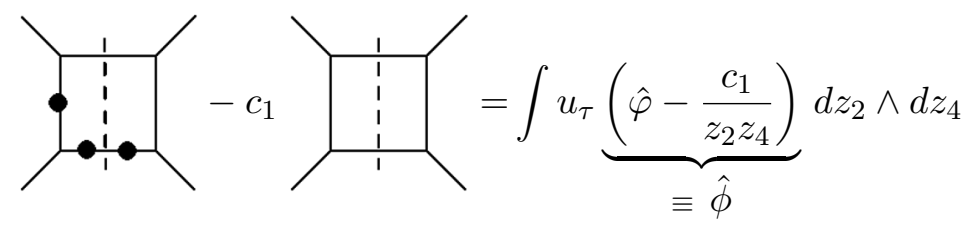


has to be reducible to the $s$-channel bubble. This property is not manifest because $\hat{\phi}$ contains a double pole in $z_{2}$ and a simple pole in $z_{4}$ which do not belong to the $s$-bubble sector. However, by exploiting the equivalence class properties, $\phi$ can be made equivalent to a form $\phi^{\prime}$ free of these poles. Accordingly, we define $\phi^{\prime} \sim \phi$, such as

$$
\phi^{\prime} \equiv \phi-\nabla_{\omega} \xi
$$

with the following ansatz for $\xi$,

$$
\xi=\frac{\sum_{i=-1, j=-1}^{2,2} \kappa_{1, i, j} z_{2}^{i} z_{4}^{j} \mathrm{~d} z_{4}+\sum_{i=-2, j=0}^{2,2} \kappa_{2, i, j} z_{2}^{i} z_{4}^{j} \mathrm{~d} z_{2}}{\mathcal{B}_{\tau}} .
$$

Fitting the free coefficients $\kappa$ with the requirement that all poles of $\phi^{\prime}$ in $z_{2}$ or $z_{4}$ vanish, gives a solution

$$
\begin{aligned}
\kappa_{1,-1,-1} & =\frac{-(d-6)(d-5) t^{2}}{2 s}, & \kappa_{1,-1,0} & =\frac{(d-6)(d-5) t}{2 s}, \\
\kappa_{1,-1,1} & =0, & \kappa_{1,-1,2} & =0, \\
\kappa_{1,0,-1} & =\frac{\left(3 d^{2}-36 d+107\right) t}{2 s}, & \kappa_{1,1,-1} & =\frac{-(d-7)(3 d-17)}{2 s}, \\
\kappa_{1,2,-1} & =\frac{(d-7)(d-6)}{2 s t}, & \kappa_{2,-2,0} & =\frac{-(d-5) t^{2}}{2 s}, \\
\kappa_{2,-2,1} & =\frac{(d-5) t}{2 s}, & \kappa_{2,-2,2} & =0, \\
\kappa_{2,-1,0} & =\frac{t\left(71 s-24 d s+2 d^{2} s+35 t-12 d t+d^{2} t\right)}{s^{2}}, & \kappa_{2,-1,1} & =\frac{-(d-7)(3 d-17)}{2 s}, \\
\kappa_{2,-1,2} & =\frac{(d-7)(d-6)}{2 s t}, & \kappa_{\text {remain. }} & =0 .
\end{aligned}
$$

The corresponding $\hat{\phi}^{\prime}$ is of the form

$$
\hat{\phi}^{\prime}=\frac{\mathcal{P}\left(z_{2}, z_{4}\right)}{\mathcal{B}_{\tau}^{2}},
$$

where $\mathcal{P}$ is a polynomial, so we see explicitly that the $z_{2}$ and $z_{4}$ poles are gone, and that no poles are present in $\phi$ that are not poles of $\omega$. With this we may compute the intersection number for 2-forms, and we get

$$
c_{2}=\frac{\left\langle\phi^{\prime} \mid 1\right\rangle}{\langle 1 \mid 1\rangle}=\frac{2(d-7)(d-5)(d-3)}{s^{4} t}
$$

in agreement with eqs. (5.17). The expressions for the two intersection numbers are listed in appendix C.1, and please note that they are much simpler than the one computed in the other two approaches, due to the absence of the regulator.

For the $t$-channel cut one may proceed likewise, and extract the coefficient of the $t$-channel bubble, again in agreement with eqs. (5.17).

Let us note that one could use the subtraction

$$
\hat{\phi}=\hat{\varphi}-\frac{\kappa_{1}}{z_{2} z_{4}}
$$




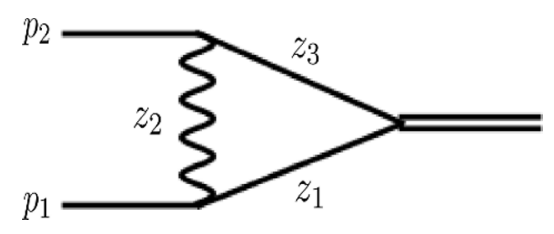

Figure 2. QED triangle.

in eq. (5.34), where $\kappa_{1}$ is a free coefficient. Then, the fitting of the unknown coefficients of eq. (5.36) generates a system whose solution does require the value $\kappa_{1}=c_{1}$. In other words, $\kappa_{1}$, which in this case corresponds to the coefficient of a master integral in the higher sector (the box function) may be fixed together with the remaining $\kappa$-parameters. ${ }^{4}$

Discussion. Considering the three methods for the intersection-based reduction of the one-loop massless box, we observe that the straight decomposition required the computation of 12 intersection numbers for 4-forms, the bottom-up decomposition required 12 intersection numbers for 2 -forms, and the top-down decomposition required 4 intersection numbers for 2 -forms. Due to the recursive nature of the multivariate algorithm of section 3 , the computation of intersection numbers for 2 -forms is much less demanding than the one of 4-forms, thereby showing the efficiency of the bottom-up algorithm compared to the straight decomposition. On the other hand, in the top-down decomposition, we compute fewer intersection numbers than in the other two approaches. Neverthless, within this approach, an extra effort is taken by the fit of the extra $\kappa$-coefficients appearing in the subtraction term, see eq. (5.35) for the one-loop massless box, which might become computationally expensive in a generic case.

\subsection{One-loop QED triangle}

In this subsection we discuss the one-loop QED triangle [figure 2]. The denominators are

$$
D_{1}=k^{2}-m^{2}, \quad D_{2}=\left(k+p_{1}\right)^{2}, \quad D_{3}=\left(k+p_{1}+p_{2}\right)^{2}-m^{2}
$$

and the kinematics is such that $p_{1}^{2}=p_{2}^{2}=m^{2},\left(p_{1}+p_{2}\right)^{2}=s$. The Baikov parametrization yields:

$$
u=\mathcal{B}^{(d-4) / 2}
$$

with

$$
\mathcal{B}=m^{2}\left(4 s z_{2}-\left(z_{1}-z_{3}\right)^{2}\right)-s\left(s z_{2}+\left(z_{1}-z_{2}\right)\left(z_{3}-z_{2}\right)\right)
$$

Performing the sector-by-sector analysis described in the beginning of section 4 we obtain $\nu_{\sigma}=1$ for the sectors

$$
\sigma \in\{\{1,3\},\{1\},\{3\}\}
$$

and $\nu_{\sigma}=0$ for the remaining ones.

\footnotetext{
${ }^{4}$ In principle such a procedure generalises beyond this example, to cases where more masters are present in the higher sectors.
} 
The master integrals are chosen as:

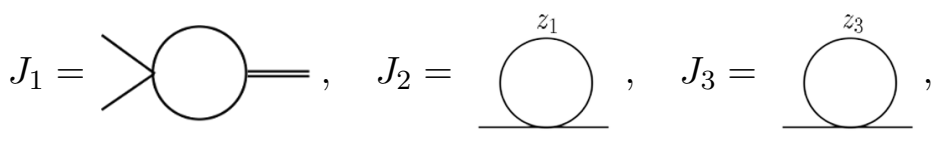

and the corresponding differential forms read

$$
\hat{e}_{1}=\frac{1}{z_{1} z_{3}}, \quad \hat{e}_{2}=\frac{1}{z_{1}}, \quad \hat{e}_{3}=\frac{1}{z_{3}} .
$$

In the following we will decompose:

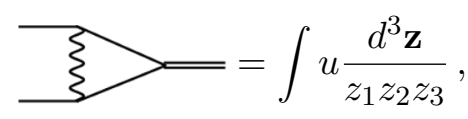

which can be expressed in terms of the chosen master integrals as

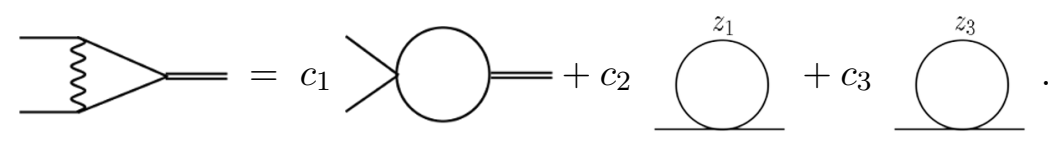

\subsubsection{Straight decomposition}

We introduce a regularized $u$ given by

$$
u_{\rho}=u \times z_{1}^{\rho} z_{2}^{\rho} z_{3}^{\rho}
$$

and then

$$
\omega_{\rho}=\sum_{i=1}^{3} \hat{\omega}_{i} d z_{i} \quad \text { with } \quad \hat{\omega}_{i}=\partial_{z_{i}} \log u_{\rho} .
$$

We consider the ordering of the variables, from the innermost to the outermost layer, as $z_{3}, z_{1}, z_{2}$ and the dimension of the twisted cohomology groups are

$$
\nu_{\{312\}}=3, \quad \nu_{\{31\}}=4, \quad \nu_{\{3\}}=2 .
$$

Given the order of variables considered above, we chose the basis elements to be

$$
\hat{e}^{(312)}=\hat{e}=\left\{\frac{1}{z_{1} z_{3}}, \frac{1}{z_{1}}, \frac{1}{z_{3}}\right\}, \quad \hat{e}^{(31)}=\left\{\frac{1}{z_{1}}, \frac{1}{z_{1} z_{3}}, \frac{1}{z_{3}}, 1\right\}, \quad \hat{e}^{(3)}=\left\{\frac{1}{z_{3}}, 1\right\},
$$

while the dual basis elements are chosen as $\hat{h}_{i}=\hat{e}_{i}$.

The required intersection numbers are

$$
\mathbf{C}_{i j}=\left\langle e_{i} \mid h_{j}\right\rangle, \quad 1 \leq i, j \leq 3
$$

and

$$
\left\langle\varphi \mid h_{k}\right\rangle, \quad 1 \leq k \leq 3
$$

Explicit expressions for the individual intersection numbers, up to the leading order in $\rho$, are presented in appendix C.2. 


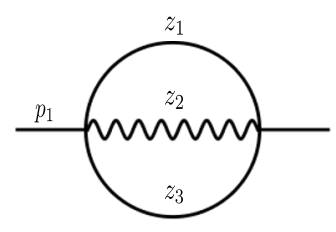

Figure 3. QED Sunrise.

Combining the intersection numbers, and taking the $\rho \rightarrow 0$ limit as in eq. (4.10), we obtain

$$
\begin{aligned}
c_{1} & =\frac{2(d-3)}{(d-4)\left(4 m^{2}-s\right)}, & c_{2}=\frac{2-d}{2(d-4) m^{2}\left(4 m^{2}-s\right)}, \\
c_{3} & =\frac{2-d}{2(d-4) m^{2}\left(4 m^{2}-s\right)} . &
\end{aligned}
$$

These coefficients are in agreement with the result obtained from FIRE [73] (before applying any symmetry relations).

\subsection{Two-loop QED sunrise}

Here, we consider 2-loop QED sunrise diagram as shown in figure 3. The denominators are:

$$
D_{1}=z_{1}=k_{1}^{2}-m^{2}, \quad D_{2}=z_{2}=\left(k_{1}-k_{2}\right)^{2}, \quad D_{3}=z_{3}=\left(k_{2}-p_{1}\right)^{2}-m^{2},
$$

while the ISPs are chosen as:

$$
z_{4}=k_{2}^{2}-m^{2}, \quad z_{5}=\left(k_{1}-p_{1}\right)^{2}-m^{2} .
$$

The Baikov parametrization gives:

$$
u(\mathbf{z})=\frac{1}{s} \mathcal{B}^{\gamma},
$$

where

$$
\begin{aligned}
\mathcal{B}=\frac{-1}{4 s}( & m^{2}\left(\left(z_{1}+z_{3}-z_{4}-z_{5}\right)^{2}-4 s z_{2}\right) \\
& -s\left(\left(z_{1}-z_{4}\right)\left(z_{3}-z_{5}\right)+z_{2}\left(z_{1}+z_{3}+z_{4}+z_{5}\right)-z_{2}^{2}\right)+s^{2} z_{2} \\
& \left.+\left(z_{1}+z_{3}-z_{4}-z_{5}\right)\left(z_{1} z_{3}-z_{4} z_{5}\right)-z_{2}\left(z_{3}-z_{4}\right)\left(z_{1}-z_{5}\right)\right)
\end{aligned}
$$

We choose the invariant $p_{1}^{2}=s$ and normalise it by the squared internal mass effect $m^{2}$, effectively setting $m^{2}=1$, and the $m^{2}$ dependence can be recovered later by power counting. We perform the sector-by-sector analysis for each of the $7\left(=2^{3}-1\right)$ sectors as described in section 4 , and obtain zero MIs in all sectors except for

$$
\sigma \in\{\{1,2,3\},\{1,3\}\}
$$


where for the sector $\{1,2,3\}$ we obtain 3 MIs and for the sector $\{1,3\} 1 \mathrm{MI}$, amounting to a total of 4 MIs. The MIs are chosen as the following:

$$
J_{1}=\mathrm{amp}^{z_{4}}, J_{2}=J^{z_{5}}, J_{4}=\bigcirc \text {, }
$$

and the corresponding differential forms read

$$
\hat{e}_{1}=\frac{1}{z_{1} z_{2} z_{3}}, \quad \hat{e}_{2}=\frac{z_{4}}{z_{1} z_{2} z_{3}}, \quad \hat{e}_{3}=\frac{z_{5}}{z_{1} z_{2} z_{3}}, \quad \hat{e}_{4}=\frac{1}{z_{1} z_{3}} .
$$

Here, we will build the differential equation for the set of master integrals

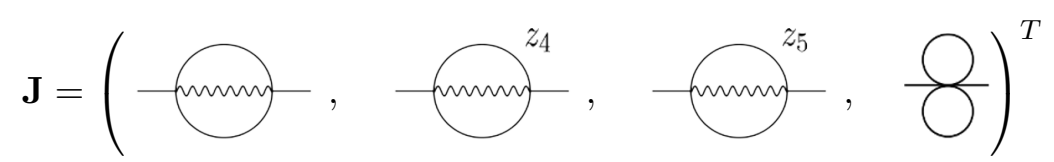

namely

$$
\partial_{s} \mathbf{J}=\mathbf{\Omega} \mathbf{J} .
$$

We will now determine $\boldsymbol{\Omega}$ using the bottom-up decomposition as described in section 4 .

\subsubsection{Bottom-up decomposition}

First we identify a spanning set of cuts $\tau$. That set is easily seen to only contain the cut corresponding to the double tadpole:

$$
\tau \in\{\{1,3\}\} .
$$

On this specific cut, we use:

$$
u_{\rho, \tau}=\frac{1}{s} z_{2}^{\rho} \mathcal{B}_{\tau}^{\gamma}
$$

with

$$
\mathcal{B}_{\tau}=\frac{1}{4 s}\left(\left(z_{5}+z_{4}-z_{2}-s\right)\left(s z_{2}-z_{4} z_{5}\right)+4 s z_{2}-\left(z_{4}+z_{5}\right)^{2}\right)
$$

and $\omega_{\rho, \tau}=\hat{\omega}_{2} \mathrm{~d} z_{2}+\hat{\omega}_{4} \mathrm{~d} z_{4}+\hat{\omega}_{5} \mathrm{~d} z_{5}$ with

$$
\hat{\omega}_{2}=\partial_{z_{2}} \log u_{\rho, \tau}, \quad \hat{\omega}_{4}=\partial_{z_{4}} \log u_{\rho, \tau}, \quad \hat{\omega}_{5}=\partial_{z_{5}} \log u_{\rho, \tau} .
$$

We consider the ordering of the variables, from the innermost to the outermost, as $z_{4}, z_{2}, z_{5}$ and the corresponding numbers of independent forms read:

$$
\nu_{\{425\}}=4, \quad \nu_{\{42\}}=2, \quad \nu_{\{4\}}=1 .
$$

On the cut we have

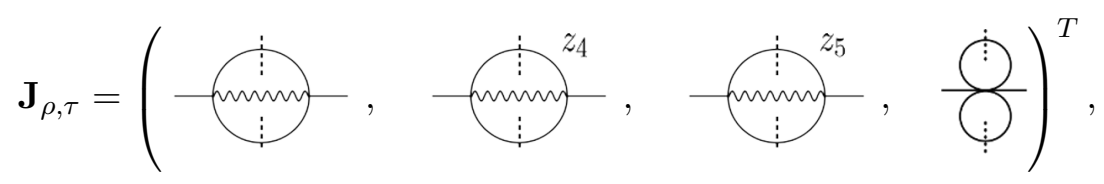


where $\mathbf{J}_{\rho, \tau}=\int_{\mathcal{C}} u_{\rho, \tau} \boldsymbol{e}_{\tau}$ and the differential equation reads

$$
\partial_{s} \mathbf{J}_{\rho, \tau}=\boldsymbol{\Omega}_{\rho} \mathbf{J}_{\rho, \tau} .
$$

The twisted cocycles corresponding to the individual MIs on the cut are

$$
\hat{e}_{\tau}^{(425)}=\hat{e}_{\tau}=\left\{\frac{1}{z_{2}}, \frac{z_{4}}{z_{2}}, \frac{z_{5}}{z_{2}}, 1\right\} .
$$

Following eq. (2.41) we define $\sigma=\partial_{s} \log u_{\rho, \tau}$ and the corresponding twisted cocycles for the decomposition of eq. (5.72) read:

$$
\hat{\varphi}_{\tau}=\left(\partial_{s}+\sigma\right) \hat{e}_{\tau}=\left\{\sigma \frac{1}{z_{2}}, \sigma \frac{z_{4}}{z_{2}}, \sigma \frac{z_{5}}{z_{2}}, \sigma\right\}
$$

For the inner spaces, we choose the basis elements as:

$$
\hat{e}^{(42)}=\left\{1, \frac{1}{z_{2}}\right\}, \quad \hat{e}^{(4)}=\{1\},
$$

and the dual basis elements are chosen as $\hat{h}_{i}=\hat{e}_{i}$.

Then, we compute the metric matrix defined as

$$
\mathbf{C}_{i j}=\left\langle e_{i, \tau} \mid h_{j, \tau}\right\rangle, \quad 1 \leq i, j \leq 4
$$

and the individual projections

$$
\left\langle\varphi_{k, \tau} \mid e_{l, \tau}\right\rangle, \quad 1 \leq k, l \leq 4 .
$$

Using eq. (3.47) we may then get the individual entries of the differential equation matrix

$$
\left(\boldsymbol{\Omega}_{\rho}\right)_{i j}=\sum_{k=1}^{4}\left\langle\varphi_{i} \mid e_{k, \tau}\right\rangle\left(\mathbf{C}^{-1}\right)_{k j}, \quad 1 \leq i, j \leq 4 .
$$

The individual multivariate intersection numbers are provided in appendix C.3. Using these intersection numbers, we obtain after taking the limit $\rho \rightarrow 0$

$$
\boldsymbol{\Omega}=\left(\begin{array}{cccc}
\frac{2 d(s-1)-5 s+6}{(s-4) s} & -\frac{3(d-2)}{2(s-4) s} & -\frac{3(d-2)}{2(s-4) s} & \frac{d-2}{(s-4) s} \\
\frac{d-2}{2} & 0 & -\frac{d-2}{2 s} & 0 \\
\frac{d-2}{2} & -\frac{d-2}{2 s} & 0 & 0 \\
0 & 0 & 0 & 0
\end{array}\right),
$$

which is in agreement with the result obtained from LiTERED [74].

\subsection{Further examples}

In the following, we present the key information useful to perform the reduction by means of intersection theory, in a set of cases all corresponding to physically relevant Feynman integrals. In particular, for each case, we provide a table containing: the definition of the integral family; the spanning cuts $(\tau)$; the dimensions of the vector spaces at each step of the recursive algorithm $(\nu)$ and the corresponding bases $(e)$, for the evaluation of multivariate intersection numbers; a pictorial decomposition of a generic integral, whose coefficients can be determined by means of our master decomposition formula eq. (2.31). In all these cases, the reduction and/or the differential equations were computed successfully, in agreement with the results of public IBP codes [73-76]. 


\section{Box with four different masses.}

\begin{tabular}{|c|c|}
\hline Integral family & Denominators \\
\hline $\begin{array}{l}z_{2} \\
s=\left(p_{1}+p_{2}\right)^{2}, \quad t=\left(p_{2}+p_{3}\right)^{2}\end{array}$ & $\begin{array}{c}z_{1}=k^{2}-m_{1}^{2} \\
z_{2}=\left(k+p_{1}\right)^{2}-m_{2}^{2} \\
z_{3}=\left(k+p_{1}+p_{2}\right)^{2}-m_{3}^{2} \\
z_{4}=\left(k+p_{1}+p_{2}+p_{3}\right)^{2}-m_{4}^{2}\end{array}$ \\
\hline
\end{tabular}

\begin{tabular}{|c|c|c|}
\hline$\tau$ & $\nu$ & $e$ \\
\hline$z_{4}=0$ & $\nu_{\{3\}}=2$ & $e^{(3)}=\left\{1, \frac{1}{z_{3}}\right\}$ \\
& $\nu_{\{32\}}=3$ & $e^{(32)}=\left\{\frac{1}{z_{2}}, \frac{1}{z_{3}}, \frac{1}{z_{2} z_{3}}\right\}$ \\
$\nu_{\{321\}}=6$ & $e^{(321)}=\left\{1, \frac{1}{z_{2}}, \frac{1}{z_{1} z_{2}}, \frac{1}{z_{1} z_{3}}, \frac{1}{z_{2} z_{3}}, \frac{1}{z_{1} z_{2} z_{3}}\right\}$ \\
\hline$z_{3}=0$ & $\nu_{\{4\}}=2$ & $e^{(4)}=\left\{1, \frac{1}{z_{4}}\right\}$ \\
$z_{241\}}=3$ & $e^{(41)}=\left\{\frac{1}{z_{1}}, \frac{1}{z_{4}}, \frac{1}{z_{1} z_{4}}\right\}$ \\
\hline$z_{2}=0$ & $\nu_{\{412\}}=6$ & $e^{(412)}=\left\{1, \frac{1}{z_{1}}, \frac{1}{z_{1} z_{2}}, \frac{1}{z_{1} z_{4}}, \frac{1}{z_{2} z_{4}}, \frac{1}{z_{1} z_{2} z_{4}}\right\}$ \\
\hline$z_{143\}}=3$ & $e^{(4)}=\left\{1, \frac{1}{z_{4}}\right\}$ \\
& $\nu_{\{431\}}=6$ & $e^{(43)}=\left\{\frac{1}{z_{3}}, \frac{1}{z_{4}}, \frac{1}{z_{3} z_{4}}\right\}$ \\
& $\nu_{\{4\}}=2$ & $\left\{1, \frac{1}{z_{4}}, \frac{1}{z_{1} z_{3}}, \frac{1}{z_{1} z_{4}}, \frac{1}{z_{3} z_{4}}, \frac{1}{z_{1} z_{3} z_{4}}\right\}$ \\
\hline & $\nu_{\{43\}}=3$ & $e^{(4)}=\left\{1, \frac{1}{z_{4}}\right\}$ \\
& $\nu_{\{432\}}=6$ & $e^{(432)}=\left\{1, \frac{1}{z_{3}}, \frac{1}{z_{2} z_{3}}, \frac{1}{z_{2} z_{4}}, \frac{1}{z_{3} z_{4}}, \frac{1}{z_{2} z_{3} z_{4}}\right\}$ \\
\hline
\end{tabular}

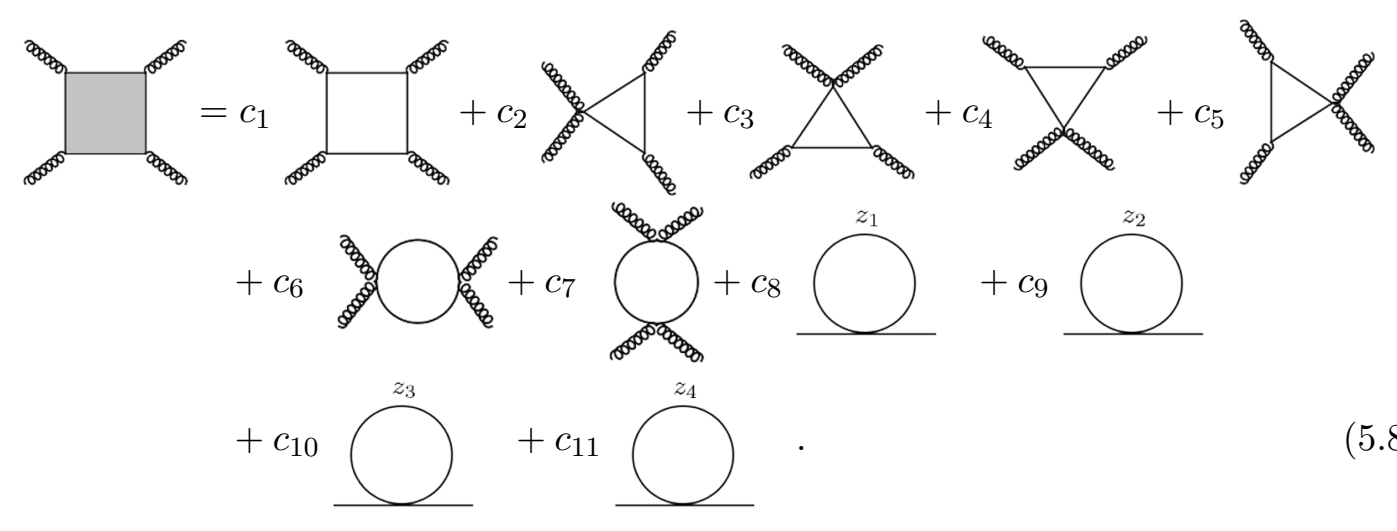


Sunrise with different masses.

\begin{tabular}{|c|c|}
\hline Integral family & Denominators \\
\hline$z_{1}$ & $z_{1}=k_{1}^{2}-m_{1}^{2}$ \\
$z_{2}$ & $z_{2}=\left(k_{1}-k_{2}\right)^{2}-m_{2}^{2}$ \\
$z_{3}=\left(k_{2}-p_{1}\right)^{2}-m_{3}^{2}$ \\
$z_{3}$ & $z_{4}=k_{2}^{2}-m_{1}^{2}$ \\
$s=p_{1}^{2}$ & $z_{5}=\left(k_{1}-p_{1}\right)^{2}-m_{3}^{2}$ \\
\hline
\end{tabular}

\begin{tabular}{|c|c|c|}
\hline$\tau$ & $\nu$ & $e$ \\
\hline$z_{1}=0$ & $\nu_{\{5\}}=1$ & $e^{(5)}=\{1\}$ \\
$z_{2}=0$ & $\nu_{\{53\}}=2$ & $e^{(53)}=\left\{1, \frac{1}{z_{3}}\right\}$ \\
& $\nu_{\{534\}}=5$ & $e^{(534)}=\left\{1, \frac{1}{z_{3}}, \frac{z_{4}}{z_{3}}, \frac{z_{5}}{z_{3}}, \frac{z_{4}^{2}}{z_{3}}\right\}$ \\
\hline$z_{1}=0$ & $\nu_{\{5\}}=1$ & $e^{(5)}=\{1\}$ \\
$z_{3}=0$ & $\nu_{\{52\}}=2$ & $e^{(52)}=\left\{1, \frac{1}{z_{2}}\right\}$ \\
\hline$z_{\{524\}}=5$ & $e^{(524)}=\left\{1, \frac{1}{z_{2}}, \frac{z_{4}}{z_{2}}, \frac{z_{5}}{z_{2}}, \frac{z_{4}^{2}}{z_{2}}\right\}$ \\
\hline$z_{3}=0$ & $\nu_{\{5\}}=1$ & $e^{(5)}=\{1\}$ \\
& $\nu_{\{51\}}=2$ & $e^{(51)}=\left\{1, \frac{1}{z_{1}}\right\}$ \\
$z_{\{514\}}=5$ & $e^{(514)}=\left\{1, \frac{1}{z_{1}}, \frac{z_{4}}{z_{1}}, \frac{z_{5}}{z_{1}}, \frac{z_{4}^{2}}{z_{1}}\right\}$ \\
\hline
\end{tabular}

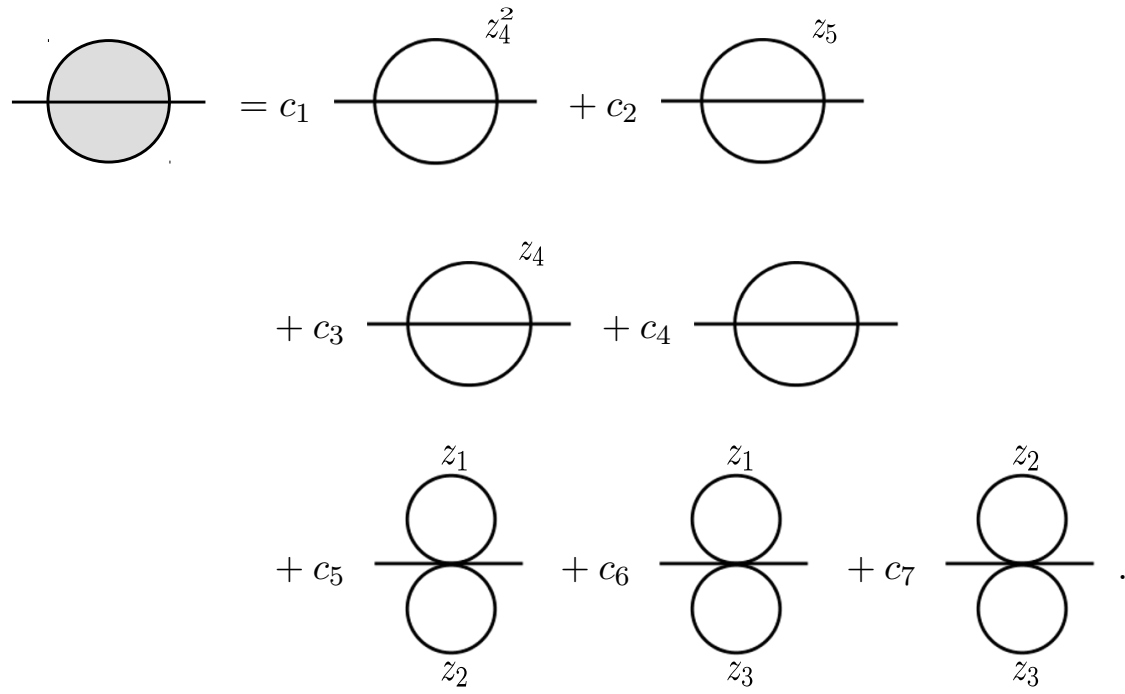


Massless planar box-triangle.

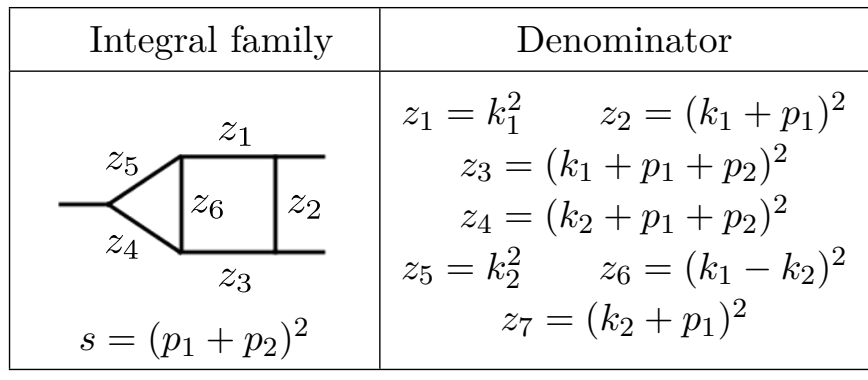

\begin{tabular}{|c|c|c|}
\hline$\tau$ & $\nu$ & $e$ \\
\hline$z_{2}=0$ & $\nu_{\{7\}}=1$ & $e^{(7)}=\{1\}$ \\
$z_{4}=0$ & $\nu_{\{73\}}=2$ & $e^{(73)}=\left\{1, \frac{1}{z_{3}}\right\}$ \\
$z_{5}=0$ & $\nu_{\{731\}}=1$ & $e^{(731)}=\{1\}$ \\
$z_{6}=0$ & & $e^{(7)}=\{1\}$ \\
\hline$z_{1}=0$ & $\nu_{\{7\}}=1$ & $e^{(76)}=\left\{\frac{1}{z_{6}}\right\}$ \\
$z_{3}=0$ & $\nu_{\{76\}}=1$ & $e^{(762)}=\{1\}$ \\
$z_{4}=0$ & $\nu_{\{762\}}=1$ & $e^{(7)}=\{1\}$ \\
$z_{5}=0$ & & $e^{(74)}=\left\{\frac{1}{z_{4}}\right\}$ \\
& $\nu_{(7)}=1$ & $e^{(742)}=\left\{\frac{1}{z_{2} z_{4}}\right\}$ \\
$z_{3}=0$ & $\nu_{\{74\}}=1$ & $e^{(7421)}=\{1\}$ \\
$z_{5}=0$ & $\nu_{\{742\}}=1$ & $e^{(7)}=\{1\}$ \\
$z_{6}=0$ & $\nu_{\{7421\}}=1$ & $e^{(75)}=\left\{\frac{1}{z_{5}}\right\}$ \\
& $\nu_{\{7\}}=1$ & $e^{(752)}=\left\{\frac{1}{z_{2} z_{5}}\right\}$ \\
$z_{1}=0$ & $\nu_{\{75\}}=1$ & $e^{(7523)}=\{1\}$ \\
$z_{4}=0$ & $\nu_{\{752\}}=1$ \\
$z_{6}=0$ & $\nu_{\{7523\}}=1$ &
\end{tabular}

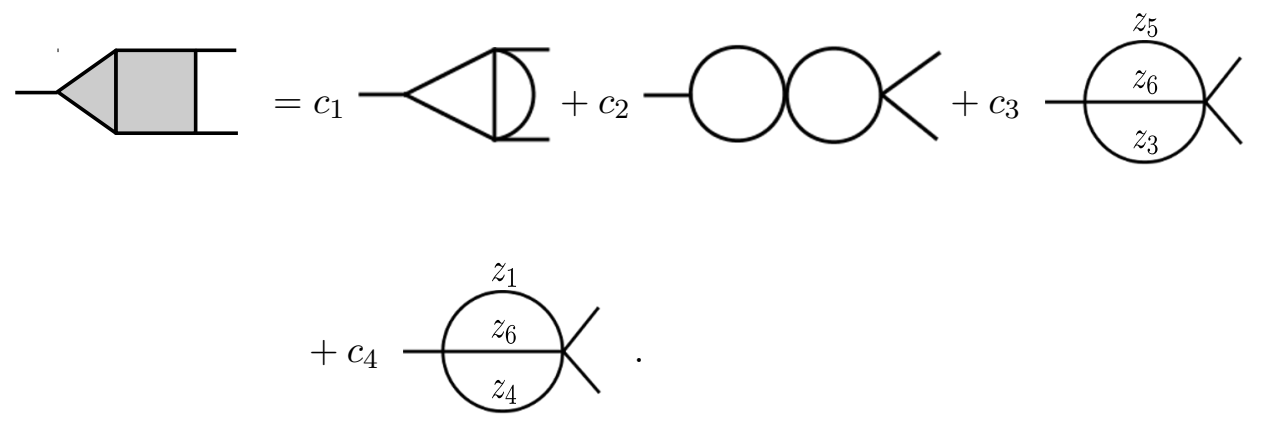


Massless non-planar triangle-box.

\begin{tabular}{|c|c|}
\hline Integral family & Denominators \\
\hline$z_{z_{6}}^{z_{1}}$ & $\begin{array}{c}z_{1}=k_{1}^{2} \\
z_{2} \\
z_{3}=\left(k_{2}+p_{1}+p_{2}\right)^{2} \\
z_{4}=k_{2}^{2} \\
z_{5}=\left(k_{1}-k_{2}\right)^{2} \\
z_{6}=\left(k_{1}-k_{2}-p_{2}\right)^{2} \\
z_{7}=\left(k_{2}+p_{1}\right)^{2}\end{array}$ \\
\hline
\end{tabular}

\begin{tabular}{|c|c|c|}
\hline$\tau$ & $\nu$ & $E$ \\
\hline $\begin{array}{l}z_{2}=0 \\
z_{3}=0 \\
z_{4}=0 \\
z_{5}=0\end{array}$ & $\begin{array}{c}\nu_{\{1\}}=2 \\
\nu_{\{16\}}=2 \\
\nu_{\{167\}}=2\end{array}$ & $\begin{array}{c}e^{(1)}=\left\{1, \frac{1}{z_{1}}\right\} \\
e^{(16)}=\left\{\frac{1}{z_{6}}, \frac{1}{z_{1} z_{6}}\right\} \\
e^{(167)}=\left\{1, \frac{1}{z_{1} z_{6}}\right\}\end{array}$ \\
\hline $\begin{array}{l}z_{1}=0 \\
z_{3}=0 \\
z_{4}=0 \\
z_{6}=0\end{array}$ & $\begin{array}{c}\nu_{\{2\}}=2 \\
\nu_{\{25\}}=2 \\
\nu_{\{257\}}=2\end{array}$ & $\begin{array}{c}e^{(2)}=\left\{1, \frac{1}{z_{2}}\right\} \\
e^{(25)}=\left\{\frac{1}{z_{5}}, \frac{1}{z_{2} z_{5}}\right\} \\
e^{(257)}=\left\{1, \frac{1}{z_{2} z_{5}}\right\}\end{array}$ \\
\hline $\begin{array}{l}z_{1}=0 \\
z_{3}=0 \\
z_{5}=0\end{array}$ & $\begin{array}{c}\nu_{\{2\}}=2 \\
\nu_{\{24\}}=2 \\
\nu_{\{246\}}=3 \\
\nu_{\{2467\}}=2\end{array}$ & $\begin{array}{c}e^{(2)}=\left\{1, \frac{1}{z_{2}}\right\} \\
e^{(24)}=\left\{\frac{1}{z_{4}}, \frac{1}{z_{2} z_{4}}\right\} \\
e^{(246)}=\left\{\frac{1}{z_{6}}, \frac{1}{z_{4} z_{6}}, \frac{1}{z_{2} z_{4} z_{6}}\right\} \\
e^{(2467)}=\left\{1, \frac{1}{z_{2} z_{4} z_{6}}\right\}\end{array}$ \\
\hline $\begin{array}{l}z_{2}=0 \\
z_{4}=0 \\
z_{6}=0\end{array}$ & $\begin{array}{c}\nu_{\{1\}}=2 \\
\nu_{\{15\}}=2 \\
\nu_{\{153\}}=3 \\
\nu_{\{1537\}}=2\end{array}$ & $\begin{array}{c}e^{(1)}=\left\{1, \frac{1}{z_{1}}\right\} \\
e^{(15)}=\left\{\frac{1}{z_{5}}, \frac{1}{z_{1} z_{5}}\right\} \\
e^{(153)}=\left\{\frac{1}{z_{3}}, \frac{1}{z_{3} z_{5}}, \frac{1}{z_{1} z_{3} z_{5}}\right\} \\
e^{(1537)}=\left\{1, \frac{1}{z_{1} z_{3} z_{5}}\right\}\end{array}$ \\
\hline
\end{tabular}

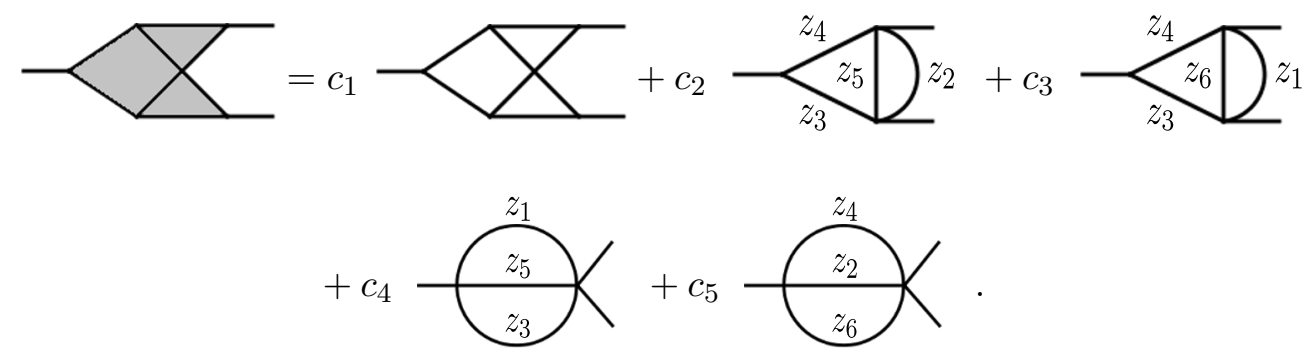




\section{Massless double-box on a triple cut.}

\begin{tabular}{|c|c|}
\hline Integral family & Denominators \\
\hline 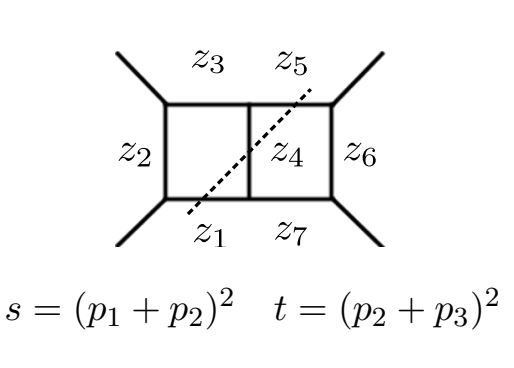 & $\begin{array}{c}z_{1}=k_{1}^{2} \quad z_{2}=\left(k_{1}-p_{1}\right)^{2} \\
z_{3}=\left(k_{1}-p_{1}-p_{2}\right)^{2} \\
z_{4}=\left(k_{1}-k_{2}\right)^{2} \\
z_{5}=\left(k_{2}-p_{1}-p_{2}\right)^{2} \\
z_{6}=\left(k_{2}-p_{1}-p_{2}-p_{3}\right)^{2} \\
z_{7}=k_{2}^{2} \quad z_{8}=\left(k_{2}-p_{1}\right)^{2} \\
z_{9}=\left(k_{1}-p_{1}-p_{2}-p_{3}\right)^{2}\end{array}$ \\
\hline
\end{tabular}

\begin{tabular}{|c|c|c|}
\hline$\tau$ & $\nu$ & $e$ \\
\hline & $\nu_{\{8\}}=1$ & $e^{(8)}=\{1\}$ \\
& $\nu_{\{87\}}=2$ & $e^{(87)}=\left\{1, \frac{1}{z_{7}}\right\}$ \\
$z_{1}=0$ & $\nu_{\{876\}}=2$ & $e^{(876)}=\left\{\frac{1}{z_{6}}, \frac{1}{z_{7}}\right\}$ \\
$z_{4}=0$ & $\nu_{\{8762\}}=4$ & $e^{(8762)}=\left\{\frac{1}{z_{2}}, \frac{1}{z_{6}}, \frac{1}{z_{7}}, \frac{1}{z_{2} z_{6}}\right\}$ \\
$z_{5}=0$ & $\nu_{\{87629\}}=5$ & $e^{(87629)}=\left\{1, \frac{1}{z_{2}}, \frac{1}{z_{6}}, \frac{1}{z_{7}}, \frac{1}{z_{2} z_{6}}\right\}$ \\
& $\nu_{\{876293\}}=4$ & $e^{(876293)}=\left\{1, \frac{1}{z_{2} z_{6}}, \frac{1}{z_{2} z_{3} z_{6} z_{7}}, \frac{z_{8}}{z_{2} z_{3} z_{6} z_{7}}\right\}$ \\
\hline
\end{tabular}

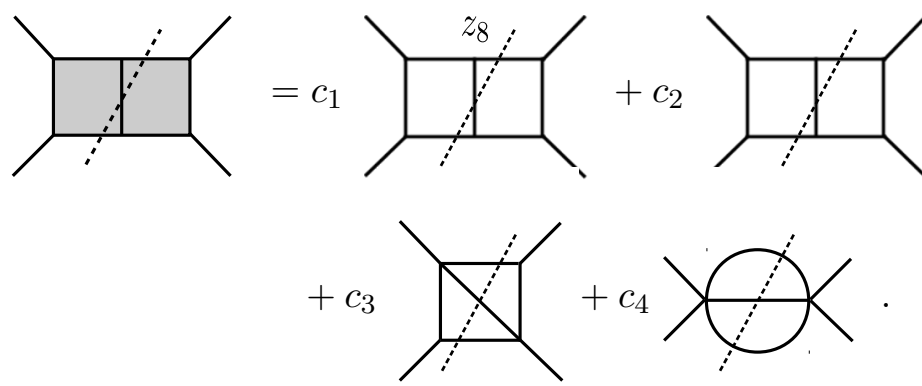

\section{Conclusions}

In this work, we elaborated on the vector space structure of Feynman integrals, and on the existence of what amounts to a scalar product among them first presented in ref. [3], showing a detailed description of their systematic decomposition in terms of Master Integrals. In particular, we described the evaluation of multivariate intersection numbers for twisted cocycles, which are the key ingredient of the master decomposition formula eq. (2.31), in terms of a recursive algorithm boiling the computations down to univariate intersection numbers. We applied the master decomposition formula to derive integral relations and differential equations for a number of Feynman integrals. As shown in previous works $[1,2]$, they can also be used for deriving dimensional recurrence relations (finitedifference equations) for Feynman integrals. We discussed algebraic properties of integrals and dual integrals as well as systems of differential equations they obey. 
We provided three different strategies for Feynman integral reduction, which we dubbed the straight decomposition, the bottom-up decomposition, and the top-down decomposition, which show possible combinations of the intersection-theory concepts together with unitarity-based methods and integrand decomposition.

The recursive computation of multivariate intersection numbers requires regulated integrals, not plagued by spurious irregular behavior which might emerge at the intermediate steps of the evaluation. For this purpose, we employed the analytic regularization procedure. On the other hand, using the richer mathematical structure of the relative twisted cohomology, the use of regulators might be avoided, thereby offering a very interesting new direction for future studies and applications to physics.

Let us conclude by observing that the decomposition formula, or better the corresponding formula for the identity resolution, in terms of multivariate intersection numbers, is applicable to generic parametric representations of Feynman integrals, including those not considered here. More generally, it can be used to derive linear and quadratic relations for Aomoto-Gel'fand type of integrals (and their duals), which are of broad interest and have applications in different contexts in physics as well as mathematics.

\section{Acknowledgments}

P.M. would like to thank Nima Arkani-Hamed, Sergio Cacciatori, Thibault Damour, Maxim Kontsevich, and Ugo Moschella for stimulating discussions. We also thank Giulio Salvatori for interesting comments. P.M. and S.M. would like to acknowledge the hospitality of the Center of Mathematical Sciences and Applications (CMSA), Harvard University, U.S.A. H.F., M.K.M. and P.M. would like to acknowledge the hospitality of the Institut des Hautes Études Scientifique (IHES), France. We would like to thank all the participants of the workshop "MathemAmplitudes 2019: Intersection Theory and Feynman Integrals" for stimulating discussions as well as for a very lively scientific environment. The work of H.F. is part of the HiProLoop project funded by the European Union's Horizon 2020 research and innovation programme under the Marie Skłodowska-Curie grant agreement 747178 . The work of M.K.M., and P.M. is supported by the Supporting TAlent in ReSearch at Padova University (UniPD STARS Grant 2017 "Diagrammalgebra"). The work of M.K.M is supported by Fellini - Fellowship for Innovation at INFN funded by the European Union's Horizon 2020 research and innovation programme under the Marie SkłodowskaCurie grant agreement No 754496. S.M. gratefully acknowledges the funding provided by Carl P. Feinberg. F.G. is supported by Fondazione Cassa di Risparmio di Padova e Rovigo (CARIPARO). The figures were drawn with Jaxodraw [77] based on Axodraw [78]. CloudVeneto is acknowledged for the use of computing and storage facilities.

\section{A Master decomposition formula}

The reduction of an integral is achieved by expressing the corresponding twisted cocycle, say $\left\langle\varphi_{L}\right|$, as a linear combination of a set of $\nu$ basic elements, $\left\langle e_{i}\right|$, referred to as the master forms. For this purpose, we introduce a dual space of twisted cocycles, generated by the 
basis $\left|h_{i}\right\rangle$ for $i=1,2, \ldots, \nu$, and define the metric matrix $\mathbf{C}_{i j} \equiv\left\langle e_{i} \mid h,\right\rangle$. Now, we can build the following $(\nu+1) \times(\nu+1)$ matrix $\mathbf{M}$, defined as,

$$
\mathbf{M}=\left(\begin{array}{ccccc}
\left\langle\varphi_{L} \mid \varphi_{R}\right\rangle & \left\langle\varphi_{L} \mid h_{1}\right\rangle & \left\langle\varphi_{L} \mid h_{2}\right\rangle & \ldots & \left\langle\varphi_{L} \mid h_{\nu}\right\rangle \\
\left\langle e_{1} \mid \varphi_{R}\right\rangle & \left\langle e_{1} \mid h_{1}\right\rangle & \left\langle e_{1} \mid h_{2}\right\rangle & \ldots & \left\langle e_{1} \mid h_{\nu}\right\rangle \\
\left\langle e_{2} \mid \varphi_{R}\right\rangle & \left\langle e_{2} \mid h_{1}\right\rangle & \left\langle e_{2} \mid h_{2}\right\rangle & \ldots & \left\langle e_{2} \mid h_{\nu}\right\rangle \\
\vdots & \vdots & \vdots & \ddots & \vdots \\
\left\langle e_{\nu} \mid \varphi_{R}\right\rangle & \left\langle e_{\nu} \mid h_{1}\right\rangle & \left\langle e_{\nu} \mid h_{2}\right\rangle & \ldots & \left\langle e_{\nu} \mid h_{\nu}\right\rangle
\end{array}\right) \equiv\left(\begin{array}{ccc}
\left\langle\varphi_{L} \mid \varphi_{R}\right\rangle & \mathbf{A}^{\boldsymbol{\top}} \\
\mathbf{B} & \mathbf{C}
\end{array}\right) .
$$

We label the columns of the matrix by $\left|\varphi_{R}\right\rangle,\left|h_{1}\right\rangle,\left|h_{2}\right\rangle, \ldots,\left|h_{\nu}\right\rangle$ for an arbitrary $\left|\varphi_{R}\right\rangle$, and the rows by $\left\langle\varphi_{L}\right|,\left\langle e_{1}\right|,\left\langle e_{2}\right|, \ldots,\left\langle e_{\nu}\right|$. The entries of this matrix are given by the pairing (bilinear) between the corresponding rows and columns. In the second equality, we express the matrix $\mathbf{M}$ as a $\nu \times \nu$ submatrix $\mathbf{C}$, a column vector $\mathbf{B}$ and a row vector $\mathbf{A}^{\top}$, respectively with elements $\mathbf{B}_{i}=\left\langle e_{i} \mid \varphi_{R}\right\rangle$ and $\mathbf{A}_{i}=\left\langle\varphi_{L} \mid h_{i}\right\rangle$ (for $i=1,2, \ldots, \nu$ ). Now, as the space of the twisted cocycles is $\nu$-dimensional and each entry of the matrix $\mathbf{M}$ is a bilinear, implies that the determinant of this matrix vanishes.

Using the well-known identity for the determinant of a block matrix, we find:

$$
\operatorname{det} \mathbf{M}=\operatorname{det} \mathbf{C}\left(\left\langle\varphi_{L} \mid \varphi_{R}\right\rangle-\mathbf{A}^{\top} \mathbf{C}^{-1} \mathbf{B}\right)=0 .
$$

Since $\operatorname{det} \mathbf{C}$ is non-zero by definition, we conclude that:

$$
\begin{aligned}
\left\langle\varphi_{L} \mid \varphi_{R}\right\rangle & =\mathbf{A}^{\top} \mathbf{C}^{-1} \mathbf{B} \\
& =\sum_{i, j=1}^{\nu}\left\langle\varphi_{L} \mid h_{j}\right\rangle\left(\mathbf{C}^{-1}\right)_{j i}\left\langle e_{i} \mid \varphi_{R}\right\rangle .
\end{aligned}
$$

This equation is very important. It can be exploited in three ways:

- Because of the arbitrariness of both $\left\langle\varphi_{L}\right|$ and $\left|\varphi_{R}\right\rangle$, the r.h.s. of the above equation implies that $\sum_{i, j=1}^{\nu}\left|h_{j}\right\rangle\left(\mathbf{C}^{-1}\right)_{j i}\left\langle e_{i}\right|$ acts like the identity operator $\mathbb{I}_{c}$ (in the cohomology space) when contracted with left and right forms,

$$
\sum_{i, j=1}^{\nu}\left|h_{j}\right\rangle\left(\mathbf{C}^{-1}\right)_{j i}\left\langle e_{i}\right| \equiv \mathbb{I}_{c} .
$$

- Because of the arbitrariness of $\left|\varphi_{R}\right\rangle$, the equation gives the decomposition of $\left\langle\varphi_{L}\right|$, as

$$
\left\langle\varphi_{L}\right|=\sum_{i=1}^{\nu} \underbrace{\sum_{j=1}^{\nu}\left\langle\varphi_{L} \mid h_{j}\right\rangle\left(\mathbf{C}^{-1}\right)_{j i}}_{c_{i}}\left\langle e_{i}\right| .
$$

- Because of the arbitrariness of $\left\langle\varphi_{L}\right|$, the equation gives the decomposition of dual forms $\left|\varphi_{R}\right\rangle$, as

$$
\left|\varphi_{R}\right\rangle=\sum_{i=1}^{\nu} \underbrace{\sum_{j=1}^{\nu}\left(\mathbf{C}^{-1}\right)_{i j}\left\langle e_{j} \mid \varphi_{R}\right\rangle}_{\widetilde{c}_{i}}\left|h_{i}\right\rangle .
$$


With a similar approach, starting with the intersection number $\left[\mathcal{C}_{L} \mid \mathcal{C}_{R}\right]$ of integration contours (homology classes), one can derive a formula analogous to eq. (A.3),

$$
\left[\mathcal{C}_{L} \mid \mathcal{C}_{R}\right]=\sum_{i, j=1}^{\nu}\left[\mathcal{C}_{L} \mid \gamma_{j}\right]\left(\mathbf{H}^{-1}\right)_{j i}\left[\delta_{i} \mid \mathcal{C}_{R}\right],
$$

where $\left.\mid \gamma_{i}\right]$ and $\left[\delta_{i} \mid\right.$ are bases of the homology space and its dual space, respectively, and $\mathbf{H}_{i j} \equiv\left[\delta_{i} \mid \gamma_{j}\right]$ is the intersection matrix of the bases elements. As done earlier for the cohomologies, eq. (A.7) can be exploited to derive the identity operator $\mathbb{I}_{h}$ in the homology space, as well as the decompositions of contours $\left[\mathcal{C}_{L} \mid\right.$ and dual contours $\left.\mid \mathcal{C}_{R}\right]$.

\section{B Derivation of the connection $\Omega$ for $n$-form intersection numbers}

Let us recall how the covariant derivative emerges in the 1 -form case, with $u=u\left(z_{1}\right)$. We consider the vanishing surface term,

$$
\begin{aligned}
0 & =\int_{\mathcal{C}_{R}} d_{z_{1}}\left(\xi_{L}\left(z_{1}\right) u\right)=\int_{\mathcal{C}_{R}}\left(d_{z_{1}} \xi_{L}+d_{z_{1}} \log u \wedge \xi_{L}\right) u \\
& \left.\equiv \int_{\mathcal{C}_{R}} \nabla_{\boldsymbol{\Omega}^{(1)}} \xi_{L} u \equiv\left\langle\nabla_{\boldsymbol{\Omega}^{(1)}} \xi_{L}\right| \mathcal{C}_{R}\right]
\end{aligned}
$$

where we defined the covariant derivative, $\nabla_{\boldsymbol{\Omega}^{(1)}} \equiv d_{z_{1}}+\boldsymbol{\Omega}^{(1)}$, using,

$$
d_{z_{1}} u=\boldsymbol{\Omega}^{(1)} u
$$

and $\boldsymbol{\Omega}^{(1)} \equiv \omega_{1}=d_{z_{1}} \log u$.

Let us extend the above derivation to the case of $n$-forms with $u=u\left(z_{1}, \ldots, z_{n}\right)$. Let us consider a multivariate integral $I$ over $n$ variables $z_{1}, \ldots, z_{n}$,

$$
\begin{aligned}
\left.\left\langle\varphi_{L}^{(\mathbf{n})}\right| \mathcal{C}_{R}^{(\mathbf{n})}\right]=\int_{\mathcal{C}_{R}^{(\mathbf{n})}} \varphi_{L}^{(\mathbf{n})}\left(z_{1}, \ldots, z_{n}\right) u & =\sum_{i=1}^{\nu_{\mathbf{n}-1}} \int_{\mathcal{C}_{R}^{(n)}} \varphi_{L, i}^{(n)}\left(z_{n}\right) \int_{\mathcal{C}_{R}^{(\mathbf{n}-\mathbf{1})}} e_{i}^{(\mathbf{n}-\mathbf{1})}\left(z_{1}, \ldots, z_{n}\right) u \\
& \left.=\sum_{i=1}^{\nu_{\mathbf{n}-1}} \int_{\mathcal{C}_{R}^{(n)}} \varphi_{L, i}^{(n)}\left(z_{n}\right)\left\langle e_{i}^{(\mathbf{n}-\mathbf{1})}\right| \mathcal{C}_{R}^{(\mathbf{n}-\mathbf{1})}\right]
\end{aligned}
$$

where we defined

$$
\left.\left\langle e_{i}^{(\mathbf{n}-\mathbf{1})}\right| \mathcal{C}_{R}^{(\mathbf{n}-\mathbf{1})}\right] \equiv \int_{\mathcal{C}_{R}^{(\mathbf{n}-\mathbf{1})}} e_{i}^{(\mathbf{n}-\mathbf{1})}\left(z_{1}, \ldots, z_{n}\right) u
$$

It it is crucial to stress that $\left.\left\langle e_{i}^{(\mathbf{n}-\mathbf{1})}\right| \mathcal{C}_{R}^{(\mathbf{n}-\mathbf{1})}\right]$ now plays the same role as $u$ in the univariate case. There could exist many forms $\varphi_{L, i}^{(n)}$ that upon integration give the same result. Let us consider the vanishing surface integral in $z_{n}$ of the $z_{n}$-derivative of $\left.\left\langle e_{i}^{(\mathbf{n}-1)}\right| \mathcal{C}_{R}^{(\mathbf{n}-\mathbf{1})}\right]$ times an arbitrary function (0-form) $\xi_{i}\left(z_{n}\right)$,

$$
\left.0=\int_{\mathcal{C}_{R}^{(n)}} d_{z_{n}}\left(\xi_{i}\left(z_{n}\right)\left\langle e_{i}^{(\mathbf{n}-\mathbf{1})}\right| \mathcal{C}_{R}^{(\mathbf{n}-\mathbf{1})}\right]\right)
$$

Let us notice that the integral $\left.\left\langle e_{i}^{(\mathbf{n}-\mathbf{1})}\right| \mathcal{C}_{R}^{(\mathbf{n}-\mathbf{1})}\right]$ satisfies the following differential equation in $z_{n}$ following section 2.5 :

$$
\left.\left.d_{z_{n}}\left\langle e_{i}^{(\mathbf{n}-\mathbf{1})}\right| \mathcal{C}_{R}^{(\mathbf{n}-\mathbf{1})}\right]=\mathbf{\Omega}_{i j}^{(n)}\left\langle e_{j}^{(\mathbf{n}-\mathbf{1})}\right| \mathcal{C}_{R}^{(\mathbf{n}-\mathbf{1})}\right]
$$


where $\boldsymbol{\Omega}^{(n)}$ is a $\nu_{\mathbf{n}-\mathbf{1}} \times \nu_{\mathbf{n}-\mathbf{1}}$ matrix. Inserting this into eq. (B.5), we obtain:

$$
\begin{aligned}
0 & \left.=\int_{\mathcal{C}_{R}^{(n)}}\left(\left(\delta_{i j} d_{z_{n}}+\boldsymbol{\Omega}_{i j}^{(n)}\right) \xi_{i}\left(z_{n}\right)\right)\left\langle e_{j}^{(\mathbf{n}-\mathbf{1})}\right| \mathcal{C}_{R}^{(\mathbf{n}-\mathbf{1})}\right] \\
& \left.=\int_{\mathcal{C}_{R}^{(n)}}\left(\left(\nabla_{\boldsymbol{\Omega}^{(n)}}\right)_{i j} \xi_{i}\left(z_{n}\right)\right)\left\langle e_{j}^{(\mathbf{n}-\mathbf{1})}\right| \mathcal{C}_{R}^{(\mathbf{n}-\mathbf{1})}\right],
\end{aligned}
$$

where the final equation defines the connection at the $n$-th integration step (after $(\mathbf{n}-\mathbf{1})$ nested integrations, on the variables $z_{1}, \ldots, z_{n-1}$ ),

$$
\nabla_{\boldsymbol{\Omega}^{(n)}} \equiv \mathbb{I} d_{z_{n}}+\boldsymbol{\Omega}^{(n)} .
$$

The matrix $\boldsymbol{\Omega}^{(n)}$ can be obtained as described in section 2.5,

$$
\begin{aligned}
\left.d_{z_{n}}\left\langle e_{i}^{(\mathbf{n}-\mathbf{1})}\right| \mathcal{C}_{R}^{(\mathbf{n}-\mathbf{1})}\right] & =d_{z_{n}} \int_{\mathcal{C}_{R}^{(\mathbf{n}-\mathbf{1})}} e_{i}^{(\mathbf{n}-\mathbf{1})}\left(z_{1}, \ldots, z_{n}\right) u \\
& =\int_{\mathcal{C}_{R}^{(\mathbf{n}-\mathbf{1})}}\left(d_{z_{n}} e_{i}^{(\mathbf{n}-\mathbf{1})}\left(z_{1}, \ldots, z_{n}\right)+d_{z_{n}} \log u \wedge e_{i}^{(\mathbf{n}-\mathbf{1})}\left(z_{1}, \ldots, z_{n}\right)\right) u \\
& =\int_{\mathcal{C}_{R}^{(\mathbf{n}-\mathbf{1})}}\left(d_{z_{n}}+\omega_{n} \wedge\right) e_{i}^{(\mathbf{n}-\mathbf{1})}\left(z_{1}, \ldots, z_{n}\right) u \\
& \left.=\left\langle\left(d_{z_{n}}+\omega_{n} \wedge\right) e_{i}^{(\mathbf{n}-\mathbf{1})}\right| \mathcal{C}_{R}^{(\mathbf{n}-\mathbf{1})}\right]
\end{aligned}
$$

where $\omega_{n} \equiv d_{z_{n}} \log u$.

The final line can be further simplified by using the master decomposition formula in eq. (2.31) as,

$$
\left\langle\left(d_{z_{n}}+\omega_{n} \wedge\right) e_{i}^{(\mathbf{n}-\mathbf{1})}\right|=\left\langle\left(d_{z_{n}}+\omega_{n} \wedge\right) e_{i}^{(\mathbf{n}-\mathbf{1})} \mid h_{k}^{(\mathbf{n}-\mathbf{1})}\right\rangle\left(\mathbf{C}_{(\mathbf{n}-\mathbf{1})}^{-1}\right)_{k j}\left\langle e_{j}^{(\mathbf{n}-\mathbf{1})}\right| .
$$

Using eq. (B.6), we can identify $\boldsymbol{\Omega}^{(n)}$ as,

$$
\boldsymbol{\Omega}_{i j}^{(n)}=\left\langle\left(d_{z_{n}}+\omega_{n} \wedge\right) e_{i}^{(\mathbf{n}-\mathbf{1})} \mid h_{k}^{(\mathbf{n}-\mathbf{1})}\right\rangle\left(\mathbf{C}_{(\mathbf{n}-\mathbf{1})}^{-1}\right)_{k j} .
$$

Dual formula. With a similar derivation, starting from the vanishing surface term,

$$
0=\int_{\mathcal{C}_{L}^{(n)}} d_{z_{n}}\left(\xi_{i}\left(z_{n}\right)\left[\mathcal{C}_{L}^{(\mathbf{n}-\mathbf{1})}\left|h_{i}^{(\mathbf{n}-\mathbf{1})}\right\rangle\right)\right.
$$

and using,

$$
d_{z_{n}}\left[\mathcal{C}_{L}^{(\mathbf{n}-\mathbf{1})}\left|h_{i}^{(\mathbf{n}-\mathbf{1})}\right\rangle=-\left[\mathcal{C}_{L}^{(\mathbf{n}-\mathbf{1})}\left|h_{j}^{(\mathbf{n}-\mathbf{1})}\right\rangle \tilde{\mathbf{\Omega}}_{j i}^{(n)}\right.\right.
$$

we obtain

$$
0=\int_{\mathcal{C}_{L}^{(n)}}\left(\nabla_{-\tilde{\mathbf{\Omega}}^{(n)}}\right)_{j i} \xi_{i}\left(z_{n}\right)\left[\mathcal{C}_{L}^{(\mathbf{n}-\mathbf{1})}\left|h_{j}^{(\mathbf{n}-\mathbf{1})}\right\rangle,\right.
$$

where the dual connection is defined as,

$$
\nabla_{-\tilde{\boldsymbol{\Omega}}^{(n)}} \equiv \mathbb{I} d_{z_{n}}-\tilde{\mathbf{\Omega}}^{(n)},
$$

with

$$
\tilde{\mathbf{\Omega}}_{j i}^{(n)}=-\left(\mathbf{C}_{(\mathbf{n}-\mathbf{1})}^{-1}\right)_{j k}\left\langle e_{k}^{(\mathbf{n}-\mathbf{1})} \mid\left(d_{z_{n}}-\omega_{n} \wedge\right) h_{i}^{(\mathbf{n}-\mathbf{1})}\right\rangle .
$$




\section{Intersection numbers for the three examples}

In this appendix we provide the explicit form of intersection numbers needed for the Feynman integral decompositions performed in section 5. Since we work in analytic regularization with a parameter $\rho$ that is taken to zero at the end of the computation, it suffices to know only the leading $\rho$-orders of intersection numbers. While our algorithm computes them exactly in $\rho$, in order to save space in this appendix we list only the leading term for each intersection number individually. One can check that the orders given here are sufficient for reconstructing the coefficients $c_{i}$ to order $\mathcal{O}\left(\rho^{0}\right)$ and that their limit as $\rho \rightarrow 0$ is in fact smooth.

\section{C.1 One-loop massless box}

\section{C.1.1 Straight decomposition}

Here we provide the intersection numbers, up to the leading order in $\rho$ required for the decomposition presented in subsection 5.1.1:

$$
\mathbf{C}_{i j}=\left\langle e_{i} \mid h_{j}\right\rangle, \quad 1 \leq i, j \leq 3
$$

with

$$
\begin{aligned}
\left\langle e_{1} \mid h_{1}\right\rangle & =\frac{1}{\rho^{4}}+\mathcal{O}\left(\rho^{-3}\right), \\
\left\langle e_{1} \mid h_{2}\right\rangle & =-\frac{s t}{(d-7)(d-6) \rho^{2}}+\mathcal{O}\left(\rho^{-1}\right), \\
\left\langle e_{1} \mid h_{3}\right\rangle & =\left\langle e_{1} \mid h_{2}\right\rangle, \\
\left\langle e_{2} \mid h_{1}\right\rangle & =-\frac{s t}{(d-4)(d-3) \rho^{2}}+\mathcal{O}\left(\rho^{-1}\right), \\
\left\langle e_{2} \mid h_{2}\right\rangle & =-\frac{s^{2} t(s+t)}{4(d-7)(d-3) \rho^{2}}+\mathcal{O}\left(\rho^{-1}\right), \\
\left\langle e_{2} \mid h_{3}\right\rangle & =-\frac{s t\left((d-4)^{2} s^{2}+((d-10) d+28) s t+(d-6)^{2} t^{2}\right)}{(d-7)(d-6)^{2}(d-4)^{2}(d-3)}+\mathcal{O}(\rho), \\
\left\langle e_{3} \mid h_{1}\right\rangle & =\left\langle e_{2} \mid h_{1}\right\rangle, \quad, \quad \\
\left\langle e_{3} \mid h_{2}\right\rangle & =-\frac{s t\left((d-6)^{2} s^{2}+((d-10) d+28) s t+(d-4)^{2} t^{2}\right)}{(d-7)(d-6)^{2}(d-4)^{2}(d-3)}+\mathcal{O}(\rho), \\
\left\langle e_{3} \mid h_{3}\right\rangle & =-\frac{s t^{2}(s+t)}{4(d-7)(d-3) \rho^{2}}+\mathcal{O}\left(\rho^{-1}\right),
\end{aligned}
$$

and

$$
\left\langle\varphi \mid h_{k}\right\rangle, \quad 1 \leq k \leq 3
$$


with

$$
\begin{aligned}
& \left\langle\varphi \mid h_{1}\right\rangle=\frac{(7-d)(d-6)(d-5)}{2 \rho^{4} s^{2} t}+\mathcal{O}\left(\rho^{-3}\right), \\
& \left\langle\varphi \mid h_{2}\right\rangle=\frac{(5-d) t}{2 \rho^{2} s^{2}}+\mathcal{O}\left(\rho^{-1}\right), \\
& \left\langle\varphi \mid h_{3}\right\rangle=-\frac{(d-5)((d-6) t+2 s)}{2(d-8) \rho^{2} t^{2}}+\mathcal{O}\left(\rho^{-1}\right) .
\end{aligned}
$$

\section{C.1.2 Bottom-up decomposition}

Here we provide the intersection numbers required for the decomposition presented in subsection 5.1.2, on the $\tau=\{1,3\}$ cut:

$$
\mathbf{C}_{i j}=\left\langle e_{i, \tau} \mid h_{j, \tau}\right\rangle, \quad 1 \leq i, j \leq 2
$$

with

$$
\begin{aligned}
\left\langle e_{1, \tau} \mid h_{1, \tau}\right\rangle & =\frac{d-5}{\rho^{2}(d-5+2 \rho)}, \\
\left\langle e_{1, \tau} \mid h_{2, \tau}\right\rangle & =\frac{-(d-5) s t}{(d-7+2 \rho)(d-6+2 \rho)(d-5+2 \rho)}, \\
\left\langle e_{2, \tau} \mid h_{1, \tau}\right\rangle & =\frac{-(d-5) s t}{(d-5+2 \rho)(d-4+2 \rho)(d-3+2 \rho)}, \\
\left\langle e_{2, \tau} \mid h_{2, \tau}\right\rangle & =\frac{(d-5) s^{2} t\left(4 \rho^{2} t-(d-6+4 \rho)(d-4+4 \rho)(s+t)\right)}{4(d-7+2 \rho)(d-6+2 \rho)(d-5+2 \rho)(d-4+2 \rho)(d-3+2 \rho)},
\end{aligned}
$$

and

$$
\left\langle\varphi_{\tau} \mid h_{k, \tau}\right\rangle, \quad 1 \leq k \leq 2
$$

with

$$
\begin{aligned}
\left\langle\varphi_{\tau} \mid h_{1, \tau}\right\rangle & =\frac{(d-5)(d-7+2 \rho)((d-6+4 \rho) s+2 \rho t)}{2(\rho-1) \rho^{2} s^{3} t}, \\
\left\langle\varphi_{\tau} \mid h_{2, \tau}\right\rangle & =\frac{(d-5) t}{2(\rho-1) s^{2}} .
\end{aligned}
$$

\section{C.1.3 Top-down decomposition}

For consistency with the straight decomposition and the bottom-up decomposition, we also provide here the intersection numbers needed for the top-down decomposition of subsection 5.1.3, on the $\tau=\{1,3\}$ cut. They are

$$
\langle\phi \mid 1\rangle=\frac{-(d-5)(s+t)}{2 s^{2}}, \quad\langle 1 \mid 1\rangle=\frac{-s^{2} t(s+t)}{4(d-7)(d-3)} .
$$

\section{C.2 One-loop QED triangle}

Here we provide the intersection numbers, up to the leading order in $\rho$, required for the system of differential equations presented in subsection 5.2.1:

$$
\mathbf{C}_{i j}=\left\langle e_{i} \mid h_{j}\right\rangle, \quad 1 \leq i, j \leq 3
$$


with

$$
\begin{aligned}
\left\langle e_{1} \mid h_{1}\right\rangle= & \frac{(d-4)\left(4 m^{2}-s\right)^{2}}{2\left(2(d-4)^{2}-2\right) \rho^{2}}+\mathcal{O}\left(\rho^{-1}\right) \\
\left\langle e_{1} \mid h_{2}\right\rangle= & \frac{s\left(4 m^{2}-s\right)\left(4(2 d-9) m^{2}-(d-4) s\right)}{4(d-6)(d-5)(d-3) \rho}+\mathcal{O}\left(\rho^{0}\right) \\
\left\langle e_{1} \mid h_{3}\right\rangle= & \left\langle e_{1} \mid h_{2}\right\rangle \\
\left\langle e_{2} \mid h_{1}\right\rangle= & \frac{s\left(4 m^{2}-s\right)\left(4(2 d-7) m^{2}-(d-4) s\right)}{4(d-5)(d-3)(d-2) \rho}+\mathcal{O}\left(\rho^{0}\right) \\
\left\langle e_{2} \mid h_{2}\right\rangle= & \frac{4 m^{4} s\left(4 m^{2}-s\right)}{\left(d^{2}-8 d+12\right) \rho}+\mathcal{O}\left(\rho^{0}\right) \\
\left\langle e_{2} \mid h_{3}\right\rangle= & \left(s \left(-64(d-5)(d-3)(3(d-8) d+44) m^{6}+16((d-8) d(6(d-8) d+173)\right.\right. \\
& \left.\left.+1236) m^{4} s-16(d-6)(d-4)^{2}(d-2) m^{2} s^{2}+(d-6)(d-4)^{2}(d-2) s^{3}\right)\right) / \\
& \left(4(d-6)^{2}(d-5)(d-4)(d-3)(d-2)^{2}\right)+\mathcal{O}\left(\rho^{1}\right), \\
\left\langle e_{3} \mid h_{1}\right\rangle= & \left\langle e_{2} \mid h_{1}\right\rangle \\
\left\langle e_{3} \mid h_{2}\right\rangle= & \left\langle e_{2} \mid h_{3}\right\rangle \\
\left\langle e_{3} \mid h_{3}\right\rangle= & \left\langle e_{2} \mid h_{2}\right\rangle
\end{aligned}
$$

and

$$
\left\langle\varphi \mid h_{k}\right\rangle, \quad 1 \leq k \leq 3
$$

with

$$
\begin{aligned}
\left\langle\varphi \mid h_{1}\right\rangle & =\frac{4 m^{2}-s}{2(d-5) \rho^{2}}+\mathcal{O}\left(\rho^{-1}\right), \\
\left\langle\varphi \mid h_{2}\right\rangle & =\frac{s\left(4 m^{2}-s\right)}{2(d-6)(d-5) \rho}+\mathcal{O}\left(\rho^{0}\right), \\
\left\langle\varphi \mid h_{3}\right\rangle & =\left\langle\varphi \mid h_{2}\right\rangle .
\end{aligned}
$$

\section{C.3 Two-loop QED sunrise}

Here we provide the intersection numbers, up to the leading order in $\rho$, required for the system of differential equations presented in subsection 5.3.1:

$$
\mathbf{C}_{i j}=\left\langle e_{i, \tau} \mid h_{j, \tau}\right\rangle, \quad 1 \leq i, j \leq 4
$$

with (we use $\gamma=\frac{d-4}{2}$ )

$$
\begin{aligned}
\left\langle e_{1, \tau} \mid h_{1, \tau}\right\rangle= & \frac{\gamma^{2}(s(s((s-28) s-102)+176)-128)+8(s-1)^{2}}{3\left(81 \gamma^{4}-45 \gamma^{2}+4\right) \rho}+\mathcal{O}\left(\rho^{0}\right), \\
\left\langle e_{1, \tau} \mid h_{2, \tau}\right\rangle= & \left(( s - 1 ) \left(\gamma^{3}(s+8)(s((s-39) s+48)-64)-\gamma^{2}(s+6)(s((s-39) s+48)\right.\right. \\
& -64)-2 \gamma(s-1)((s-14) s+16)-12((s-2) s+2))) /(9(\gamma-1)(3 \gamma-2) \\
& (3 \gamma-1)(3 \gamma+1)(3 \gamma+2) \rho)+\mathcal{O}\left(\rho^{0}\right), \\
\left\langle e_{1, \tau} \mid h_{3, \tau}\right\rangle= & \left\langle e_{1} \mid h_{2}\right\rangle,
\end{aligned}
$$




$$
\begin{aligned}
& \left\langle e_{1, \tau} \mid h_{4, \tau}\right\rangle=\left(\gamma^{4}(s-4)(s(s((s-34) s-894)-544)+256)\right. \\
& -\gamma^{3}(s(s(s((s-46) s-396)+3560)+2752)-768) \\
& +2 \gamma^{2}(s(268-(s-24) s(4 s+17))+32)+12 \gamma(s(16-3(s-8) s)-4) \\
& -48 s(2 s+1)) /\left(18(\gamma-1)^{2} \gamma\left(81 \gamma^{4}-45 \gamma^{2}+4\right)\right)+\mathcal{O}\left(\rho^{1}\right) \text {, } \\
& \left\langle e_{2, \tau} \mid h_{1, \tau}\right\rangle=\left(( s - 1 ) \left(\gamma^{3}(s+8)(s((s-39) s+48)-64)+\gamma^{2}(s+6)(s((s-39) s+48)\right.\right. \\
& -64)-2 \gamma(s-1)((s-14) s+16)+12((s-2) s+2))) /(9(\gamma+1)(3 \gamma-2) \\
& (3 \gamma-1)(3 \gamma+1)(3 \gamma+2) \rho)+\mathcal{O}\left(\rho^{0}\right), \\
& \left\langle e_{2, \tau} \mid h_{2, \tau}\right\rangle=\left(-72\left((s-1) s\left(s^{2}+2\right)+1\right)+\gamma^{4}(s(s(s(s((s-36) s-1563)+1516)\right. \\
& -3168)+3840)-2048)+\gamma^{2}(1280-s(s(s(s((s-36) s-915)+1108) \\
& -2232)+2544))) /\left(27\left(\gamma^{2}\left(7-9 \gamma^{2}\right)^{2}-4\right) \rho\right)+\mathcal{O}\left(\rho^{0}\right), \\
& \left\langle e_{2, \tau} \mid h_{3, \tau}\right\rangle=\left(\gamma^{4}(s(s(s(s((s-36) s+624)+1516)-3168)+3840)-2048)\right. \\
& -\gamma^{2}(s(s(s(s((s-36) s+300)+1108)-2232)+2544)-1280) \\
& +36(s(s(s(s+2)-4)+4)-2)) /\left(27\left(\gamma^{2}\left(7-9 \gamma^{2}\right)^{2}-4\right) \rho\right)+\mathcal{O}\left(\rho^{0}\right), \\
& \left\langle e_{2, \tau} \mid h_{4, \tau}\right\rangle=\left(16 \gamma^{2}\left(32 \gamma(\gamma+1)\left(8 \gamma^{2}-5\right)+9\right)+144 \gamma+(\gamma-1) \gamma^{3}(\gamma+1)^{2} s^{6}\right. \\
& -6(\gamma-1) \gamma^{2}(\gamma+1)^{2}(7 \gamma-1) s^{5}-3 \gamma(\gamma+1)(\gamma(\gamma(\gamma(445 \gamma+98)-281) \\
& -38)+16) s^{4}+16(\gamma+1)(\gamma(\gamma(\gamma(\gamma(379 \gamma-99)-277)+108)+18) \\
& -9) s^{3}+24\left(568 \gamma^{6}-503 \gamma^{4}+121 \gamma^{2}-6\right) s^{2}-48(\gamma+1)(4 \gamma-1)(4 \gamma+1) \\
& (\gamma(\gamma(14 \gamma-5)-8)+3) s) /\left(54 \gamma\left(\gamma^{2}-1\right)^{2}\left(81 \gamma^{4}-45 \gamma^{2}+4\right)\right)+\mathcal{O}\left(\rho^{1}\right), \\
& \left\langle e_{3, \tau} \mid h_{1, \tau}\right\rangle=\left\langle e_{2} \mid h_{1}\right\rangle \text {, } \\
& \left\langle e_{3, \tau} \mid h_{2, \tau}\right\rangle=\left\langle e_{2} \mid h_{3}\right\rangle \text {, } \\
& \left\langle e_{3, \tau} \mid h_{3, \tau}\right\rangle=\left\langle e_{2} \mid h_{2}\right\rangle \text {, } \\
& \left\langle e_{3, \tau} \mid h_{4, \tau}\right\rangle=\left\langle e_{2} \mid h_{4}\right\rangle \text {, } \\
& \left\langle e_{4, \tau} \mid h_{1, \tau}\right\rangle=\left(\gamma^{4}(s-4)(s(s((s-34) s-894)-544)+256)\right. \\
& +\gamma^{3}(s(s(s((s-46) s-396)+3560)+2752)-768) \\
& +2 \gamma^{2}(s(268-(s-24) s(4 s+17))+32)+12 \gamma(s(3(s-8) s-16)+4) \\
& -48 s(2 s+1)) /\left(18 \gamma(\gamma+1)^{2}\left(81 \gamma^{4}-45 \gamma^{2}+4\right)\right)+\mathcal{O}\left(\rho^{1}\right), \\
& \left\langle e_{4, \tau} \mid h_{2, \tau}\right\rangle=\left(16(\gamma-1) \gamma(4 \gamma-3)(4 \gamma-1)(4 \gamma+1)(4 \gamma+3)+(\gamma-1)^{2} \gamma^{3}(\gamma+1) s^{6}\right. \\
& -6(\gamma-1)^{2} \gamma^{2}(\gamma+1)(7 \gamma+1) s^{5}-3(\gamma-1) \gamma(\gamma(\gamma(\gamma(445 \gamma-98)-281) \\
& +38)+16) s^{4}+16(\gamma-1)(\gamma(\gamma(\gamma(\gamma(379 \gamma+99)-277)-108)+18) \\
& +9) s^{3}+24\left(568 \gamma^{6}-503 \gamma^{4}+121 \gamma^{2}-6\right) s^{2}-48(\gamma-1)(4 \gamma-1)(4 \gamma+1) \\
& (\gamma(\gamma(14 \gamma+5)-8)-3) s) /\left(54 \gamma\left(\gamma^{2}-1\right)^{2}\left(81 \gamma^{4}-45 \gamma^{2}+4\right)\right)+\mathcal{O}\left(\rho^{1}\right), \\
& \left\langle e_{4, \tau} \mid h_{3, \tau}\right\rangle=\left\langle e_{4} \mid h_{2}\right\rangle \text {, } \\
& \left\langle e_{4, \tau} \mid h_{4, \tau}\right\rangle=\frac{2\left(4 \gamma^{2}-1\right) s^{2}}{\gamma\left(\gamma^{2}-1\right)^{2}}+\mathcal{O}\left(\rho^{1}\right),
\end{aligned}
$$

and

$$
\left\langle\varphi_{k, \tau} \mid h_{l, \tau}\right\rangle, \quad 1 \leq k, l \leq 4
$$


with

$$
\begin{aligned}
& \left\langle\varphi_{1, \tau} \mid h_{1, \tau}\right\rangle=\left(\gamma^{2}\left(s^{4}-14 s^{3}-88 s+128\right)+2 \gamma^{3}(s-1)(s((s-21) s-24)-64)\right. \\
& \left.+4 \gamma(s-2)(s-1)^{2}+8(s-1)\right) /\left(3\left(81 \gamma^{4}-45 \gamma^{2}+4\right) \rho s\right)+\mathcal{O}\left(\rho^{0}\right), \\
& \left\langle\varphi_{1, \tau} \mid h_{2, \tau}\right\rangle=\left(-8(\gamma-3)+\gamma^{2}\left(s\left((s-20) s\left(s^{2}+8\right)+452\right)-416\right)+2 \gamma(s-3) s^{2}(3 s\right. \\
& -5)+\gamma^{4}(s(s(s(73-2(s-26) s)+56)-448)+512) \\
& \left.+\gamma^{3}(s-1)(s+2)(s((s-39) s+48)-64)+12(s-2) s\right) /(9 \rho((\gamma-1)(3 \gamma \\
& -2)(3 \gamma-1)(3 \gamma+1)(3 \gamma+2) s))+\mathcal{O}\left(\rho^{0}\right), \\
& \left\langle\varphi_{1, \tau} \mid h_{3, \tau}\right\rangle=\left\langle\varphi_{1} \mid h_{2}\right\rangle \text {, } \\
& \left\langle\varphi_{1, \tau} \mid h_{4, \tau}\right\rangle=\left(2 \gamma^{5}(s-1)(s+8)(s((s-39) s+48)-64)\right. \\
& +\gamma^{4}(s(448-s(s((s-56) s-14)+1988))+256) \\
& +\gamma^{3}(s(s(s(154-(s-20) s)+640)+1720)-832) \\
& -2 \gamma^{2}(s(s(s(6 s-35)-126)+408)+8)-12 \gamma(s(s+2)(s+4)-4) \\
& +48 s) /\left(18(\gamma-1)^{2} \gamma\left(81 \gamma^{4}-45 \gamma^{2}+4\right) s\right)+\mathcal{O}\left(\rho^{1}\right) \text {, } \\
& \left\langle\varphi_{2, \tau} \mid h_{1, \tau}\right\rangle=\left(12(s-1)\left(s^{2}-2\right)+\gamma^{3}(s(s(s(2(s-26) s-73)-56)+448)-512)\right. \\
& +\gamma^{2}(s+2)(s(s(2(s-27) s-45)+208)-192)+2 \gamma(s-1)^{2}((s-2) s \\
& +16)) /\left(9\left(81 \gamma^{4}-45 \gamma^{2}+4\right) \rho s\right)+\mathcal{O}\left(\rho^{0}\right) \text {, } \\
& \left\langle\varphi_{2, \tau} \mid h_{2, \tau}\right\rangle=\left(36\left(-2 s^{4}+s^{3}-2 s+2\right)+\gamma^{4}(s(s(s(s(2(s-30) s-1323)+236)+672)\right. \\
& -2304)+2048)+6 \gamma^{3}(s-1) s(s((s-39) s+48)-64)+\gamma^{2}(s(s(s(s(855 \\
& -2(s-30) s)-392)-132)+1296)-1280)-6 \gamma(s-1) s((s-6)(s-3) s \\
& -4)) /(27(\gamma-1)(3 \gamma-2)(3 \gamma-1)(3 \gamma+1)(3 \gamma+2) \rho s)+\mathcal{O}\left(\rho^{0}\right) \text {, } \\
& \left\langle\varphi_{2, \tau} \mid h_{3, \tau}\right\rangle=\left(36\left(s^{4}+s^{3}-2 s+2\right)+2 \gamma^{4}(s(s(s(s((s-30) s+432)+118)+336)-1152)\right. \\
& +1024)+6 \gamma^{3}(s-1) s(s((s-39) s+48)-64)-2 \gamma^{2}(s(s(s(s((s-30) s+180) \\
& +196)+66)-648)+640)-6 \gamma(s-1) s((s-6)(s-3) s \\
& -4)) /(27(\gamma-1)(3 \gamma-2)(3 \gamma-1)(3 \gamma+1)(3 \gamma+2) \rho s)+\mathcal{O}\left(\rho^{0}\right) \text {, } \\
& \left\langle\varphi_{2, \tau} \mid h_{4, \tau}\right\rangle=\left(-16 \gamma(\gamma+1)(4 \gamma-3)(4 \gamma-1)(4 \gamma+1)(4 \gamma+3)+2(\gamma-1) \gamma^{3}(\gamma+1)^{2} s^{6}\right. \\
& -18(\gamma-1) \gamma^{2}(\gamma+1)^{2}(4 \gamma-1) s^{5}-3 \gamma(\gamma+1)(\gamma(\gamma(\gamma(313 \gamma+264)-273)-84) \\
& +20) s^{4}+8(\gamma+1)\left(\gamma\left(\gamma\left(379 \gamma^{3}-475 \gamma+198\right)+36\right)-18\right) s^{3}-144(\gamma-1) \gamma\left(44 \gamma^{4}\right. \\
& \left.-31 \gamma^{2}+2\right) s^{2}+48(\gamma+1)(4 \gamma-1)(4 \gamma+1)(5 \gamma-3)\left(2 \gamma^{2}\right. \\
& -1) s) /\left(54(\gamma-1)^{2} \gamma(\gamma+1)(3 \gamma-2)(3 \gamma-1)(3 \gamma+1)(3 \gamma+2) s\right)+\mathcal{O}\left(\rho^{1}\right) \text {, } \\
& \left\langle\varphi_{3, \tau} \mid h_{1, \tau}\right\rangle=\left(12(s-1)\left(s^{2}-2\right)+\gamma^{3}(s(s(s(2(s-26) s-73)-56)+448)-512)\right. \\
& +\gamma^{2}(s+2)(s(s(2(s-27) s-45)+208)-192)+2 \gamma(s-1)^{2}((s-2) s \\
& +16)) /\left(9\left(81 \gamma^{4}-45 \gamma^{2}+4\right) \rho s\right)+\mathcal{O}\left(\rho^{0}\right) \text {, }
\end{aligned}
$$




$$
\begin{aligned}
\left\langle\varphi_{3, \tau} \mid h_{2, \tau}\right\rangle= & \left(36\left(s^{4}+s^{3}-2 s+2\right)+2 \gamma^{4}(s(s(s(s((s-30) s+432)+118)+336)\right. \\
& -1152)+1024)+6 \gamma^{3}(s-1) s(s((s-39) s+48)-64)-2 \gamma^{2}(s(s(s(s(s-30) s \\
& +180)+196)+66)-648)+640)-6 \gamma(s-1) s((s-6)(s-3) s \\
& -4)) /(27(\gamma-1)(3 \gamma-2)(3 \gamma-1)(3 \gamma+1)(3 \gamma+2) \rho s)+\mathcal{O}\left(\rho^{0}\right), \\
\left\langle\varphi_{3, \tau} \mid h_{3, \tau}\right\rangle= & \left(36\left(-2 s^{4}+s^{3}-2 s+2\right)+\gamma^{4}(s(s(s(s(2(s-30) s-1323)+236)+672)\right. \\
& -2304)+2048)+6 \gamma^{3}(s-1) s(s((s-39) s+48)-64)+\gamma^{2}(s(s(s(s(855 \\
& -2(s-30) s)-392)-132)+1296)-1280)-6 \gamma(s-1) s((s-6)(s-3) s \\
& -4)) /(27(\gamma-1)(3 \gamma-2)(3 \gamma-1)(3 \gamma+1)(3 \gamma+2) \rho s)+\mathcal{O}\left(\rho^{0}\right), \\
\left\langle\varphi_{3, \tau} \mid h_{4, \tau}\right\rangle= & \left(-16 \gamma(\gamma+1)(4 \gamma-3)(4 \gamma-1)(4 \gamma+1)(4 \gamma+3)+2(\gamma-1) \gamma^{3}(\gamma+1)^{2} s^{6}\right. \\
& -18(\gamma-1) \gamma^{2}(\gamma+1)^{2}(4 \gamma-1) s^{5}-3 \gamma(\gamma+1)(\gamma(\gamma(\gamma(313 \gamma+264)-273) \\
& -84)+20) s^{4}+8(\gamma+1)\left(\gamma\left(\gamma\left(379 \gamma^{3}-475 \gamma+198\right)+36\right)-18\right) s^{3}-144(\gamma \\
& -1) \gamma\left(44 \gamma^{4}-31 \gamma^{2}+2\right) s^{2}+48(\gamma+1)(4 \gamma-1)(4 \gamma+1)(5 \gamma-3)\left(2 \gamma^{2}\right. \\
& -1) s) /\left(54(\gamma-1)^{2} \gamma(\gamma+1)(3 \gamma-2)(3 \gamma-1)(3 \gamma+1)(3 \gamma+2) s\right)+\mathcal{O}\left(\rho^{1}\right), \\
\left\langle\varphi_{4, \tau} \mid h_{3, \tau}\right\rangle= & \left\langle\varphi_{4, \tau} \mid h_{2, \tau}\right\rangle, \\
\left\langle\varphi_{4, \tau} \mid h_{4, \tau}\right\rangle= & \left(\rho \left(16 \gamma^{2}\left(32 \gamma(\gamma+1)\left(8 \gamma^{2}-5\right)+9\right)+144 \gamma+(\gamma-1) \gamma^{3}(\gamma+1)^{2} s^{6}\right.\right. \\
& -6(\gamma-1) \gamma^{2}(\gamma+1)^{2}(7 \gamma-1) s^{5}-3 \gamma(\gamma+1)(\gamma(\gamma(\gamma(445 \gamma+98)-281) \\
& -38)+16) s^{4}+16(\gamma+1)(\gamma(\gamma(\gamma(\gamma(379 \gamma-99)-277)+108)+18)-9) s^{3} \\
& +24\left(568 \gamma^{6}-503 \gamma^{4}+121 \gamma^{2}-6\right) s^{2}-48(\gamma+1)(4 \gamma-1)(4 \gamma+1)(\gamma(\gamma(14 \gamma \\
& -5)-8)+3) s)) /\left(54 \gamma\left(\gamma^{2}-1\right)^{2}\left(81 \gamma^{4}-45 \gamma^{2}+4\right) s\right)+\mathcal{O}\left(\rho^{2}\right) . \\
& -64)-2 \gamma(s-1)((s-14) s+16)+12((s-2) s+2))) /(9(\gamma+1)(3 \gamma \\
& -2)(3 \gamma-1)(3 \gamma+1)(3 \gamma+2) s)+\mathcal{O}\left(\rho^{1}\right), \\
\left\langle\varphi_{4, \tau} \mid h_{2, \tau}\right\rangle= & \left(\gamma^{4}(s(s(s(s(2(s-36) s-939)+3032)-6336)+7680)-4096)\right. \\
& \left.+\gamma_{1, \tau}^{2}(s(s(s(615-2(s-36) s)-2216)+4464)-5088)+2560\right)
\end{aligned}
$$

Open Access. This article is distributed under the terms of the Creative Commons Attribution License (CC-BY 4.0), which permits any use, distribution and reproduction in any medium, provided the original author(s) and source are credited. 


\section{References}

[1] P. Mastrolia and S. Mizera, Feynman Integrals and Intersection Theory, JHEP 02 (2019) 139 [arXiv: 1810.03818] [INSPIRE].

[2] H. Frellesvig et al., Decomposition of Feynman Integrals on the Maximal Cut by Intersection Numbers, JHEP 05 (2019) 153 [arXiv: 1901.11510] [INSPIRE].

[3] H. Frellesvig, F. Gasparotto, M.K. Mandal, P. Mastrolia, L. Mattiazzi and S. Mizera, Vector Space of Feynman Integrals and Multivariate Intersection Numbers, Phys. Rev. Lett. 123 (2019) 201602 [arXiv: 1907.02000] [INSPIRE].

[4] K. Cho and K. Matsumoto, Intersection theory for twisted cohomologies and twisted Riemann's period relations I, Nagoya Math. J. 139 (1995) 67.

[5] K. Matsumoto, Quadratic Identities for Hypergeometric Series of Type $(k, l)$, Kyushu J. Math. 48 (1994) 335.

[6] K. Matsumoto, Intersection numbers for logarithmic k-forms, Osaka J. Math. 35 (1998) 873.

[7] K. Ohara, Y. Sugiki and N. Takayama, Quadratic Relations for Generalized Hypergeometric Functions ${ }_{p} F_{p-1}$, Funkcial. Ekvac. 46 (2003) 213.

[8] Y. Goto, Twisted Cycles and Twisted Period Relations for Lauricella's Hypergeometric Function $F_{C}$, Int. J. Math. 24 (2013) 1350094 [arXiv:1308.5535].

[9] Y. Goto and K. Matsumoto, The monodromy representation and twisted period relations for Appell's hypergeometric function $F_{4}$, Nagoya Math. J. 217 (2015) 61.

[10] Y. Goto, Twisted period relations for Lauricella's hypergeometric functions $F_{A}$, Osaka J. Math. 52 (2015) 861.

[11] Y. Goto, Intersection Numbers and Twisted Period Relations for the Generalized Hypergeometric Function ${ }_{m+1} F_{m}$, Kyushu J. Math. 69 (2015) 203.

[12] S. Mizera, Scattering Amplitudes from Intersection Theory, Phys. Rev. Lett. 120 (2018) 141602 [arXiv:1711.00469] [INSPIRE].

[13] S.-J. Matsubara-Heo and N. Takayama, An algorithm of computing cohomology intersection number of hypergeometric integrals, arXiv:1904.01253.

[14] S. Mizera, Aspects of Scattering Amplitudes and Moduli Space Localization, Ph.D. Thesis, Princeton, Inst. Advanced Study (2020) [DOI] [arXiv:1906.02099] [INSPIRE].

[15] S. Abreu, R. Britto, C. Duhr, E. Gardi and J. Matthew, From positive geometries to a coaction on hypergeometric functions, JHEP 02 (2020) 122 [arXiv:1910.08358] [INSPIRE].

[16] S. Abreu, R. Britto, C. Duhr, E. Gardi and J. Matthew, Generalized hypergeometric functions and intersection theory for Feynman integrals, PoS RACOR2019 (2019) 067 [arXiv: 1912.03205] [INSPIRE].

[17] S. Mizera and A. Pokraka, From Infinity to Four Dimensions: Higher Residue Pairings and Feynman Integrals, JHEP 02 (2020) 159 [arXiv: 1910.11852] [INSPIRE].

[18] J.M. Henn, Multiloop integrals in dimensional regularization made simple, Phys. Rev. Lett. 110 (2013) 251601 [arXiv: 1304.1806] [INSPIRE].

[19] J. Chen, X. Jiang, X. Xu and L.L. Yang, Constructing canonical Feynman integrals with intersection theory, Phys. Lett. B 814 (2021) 136085 [arXiv:2008.03045] [INSPIRE]. 
[20] S. Weinzierl, On the computation of intersection numbers for twisted cocycles, arXiv:2002.01930 [INSPIRE].

[21] A. Kaderli, A note on the Drinfeld associator for genus-zero superstring amplitudes in twisted de Rham theory, J. Phys. A 53 (2020) 415401 [arXiv: 1912.09406] [INSPIRE].

[22] N. Kalyanapuram and R.G. Jha, Positive Geometries for all Scalar Theories from Twisted Intersection Theory, Phys. Rev. Res. 2 (2020) 033119 [arXiv:2006.15359] [INSPIRE].

[23] S. Weinzierl, Correlation functions on the lattice and twisted cocycles, Phys. Lett. B 805 (2020) 135449 [arXiv:2003.05839] [INSPIRE].

[24] K.G. Chetyrkin and F.V. Tkachov, Integration by Parts: The Algorithm to Calculate $\beta$-functions in 4 Loops, Nucl. Phys. B 192 (1981) 159 [INSPIRE].

[25] D. Broadhurst and A. Mellit, Perturbative quantum field theory informs algebraic geometry, PoS LL2016 (2016) 079 [INSPIRE].

[26] D. Broadhurst, Feynman integrals, L-series and Kloosterman moments, Commun. Num. Theor. Phys. 10 (2016) 527 [arXiv: 1604.03057] [INSPIRE].

[27] D. Broadhurst and D.P. Roberts, Quadratic relations between Feynman integrals, PoS LL2018 (2018) 053 [INSPIRE].

[28] Y. Zhou, Wick rotations, Eichler integrals, and multi-loop Feynman diagrams, Commun. Num. Theor. Phys. 12 (2018) 127 [arXiv:1706.08308] [INSPIRE].

[29] Y. Zhou, Wrońskian factorizations and Broadhurst-Mellit determinant formulae, Commun. Num. Theor. Phys. 12 (2018) 355 [arXiv:1711.01829] [INSPIRE].

[30] J. Fresán, C. Sabbah and J.-D. Yu, Quadratic relations between periods of connections, arXiv:2005.11525.

[31] J. Fresán, C. Sabbah and J.-D. Yu, Quadratic relations between Bessel moments, arXiv:2006.02702 [INSPIRE].

[32] R.N. Lee, Symmetric $\epsilon$ - and $(\epsilon+1 / 2)$-forms and quadratic constraints in "elliptic" sectors, JHEP 10 (2018) 176 [arXiv:1806.04846] [INSPIRE].

[33] G. Ossola, C.G. Papadopoulos and R. Pittau, Reducing full one-loop amplitudes to scalar integrals at the integrand level, Nucl. Phys. B $\mathbf{7 6 3}$ (2007) 147 [hep-ph/0609007] [InSPIRE].

[34] R.K. Ellis, W.T. Giele and Z. Kunszt, A Numerical Unitarity Formalism for Evaluating One-Loop Amplitudes, JHEP 03 (2008) 003 [arXiv: 0708.2398] [INSPIRE].

[35] R.K. Ellis, W.T. Giele, Z. Kunszt and K. Melnikov, Masses, fermions and generalized D-dimensional unitarity, Nucl. Phys. B 822 (2009) 270 [arXiv:0806.3467] [InSPIRE].

[36] P. Mastrolia, E. Mirabella and T. Peraro, Integrand reduction of one-loop scattering amplitudes through Laurent series expansion, JHEP 06 (2012) 095 [Erratum ibid. 11 (2012) 128] [arXiv: 1203.0291] [INSPIRE].

[37] Y. Zhang, Integrand-Level Reduction of Loop Amplitudes by Computational Algebraic Geometry Methods, JHEP 09 (2012) 042 [arXiv: 1205.5707] [INSPIRE].

[38] P. Mastrolia, E. Mirabella, G. Ossola and T. Peraro, Scattering Amplitudes from Multivariate Polynomial Division, Phys. Lett. B 718 (2012) 173 [arXiv:1205.7087] [INSPIRE].

[39] P. Mastrolia and G. Ossola, On the Integrand-Reduction Method for Two-Loop Scattering Amplitudes, JHEP 11 (2011) 014 [arXiv:1107.6041] [INSPIRE]. 
[40] S. Badger, H. Frellesvig and Y. Zhang, A Two-Loop Five-Gluon Helicity Amplitude in QCD, JHEP 12 (2013) 045 [arXiv: 1310.1051] [INSPIRE].

[41] Z. Bern, L.J. Dixon, D.C. Dunbar and D.A. Kosower, Fusing gauge theory tree amplitudes into loop amplitudes, Nucl. Phys. B 435 (1995) 59 [hep-ph/9409265] [InSPIRE].

[42] Z. Bern, L.J. Dixon, D.C. Dunbar and D.A. Kosower, One loop n point gauge theory amplitudes, unitarity and collinear limits, Nucl. Phys. B 425 (1994) 217 [hep-ph/9403226] [INSPIRE].

[43] R. Britto, F. Cachazo and B. Feng, Generalized unitarity and one-loop amplitudes in $N=4$ super-Yang-Mills, Nucl. Phys. B 725 (2005) 275 [hep-th/0412103] [INSPIRE].

[44] R. Britto, E. Buchbinder, F. Cachazo and B. Feng, One-loop amplitudes of gluons in SQCD, Phys. Rev. D 72 (2005) 065012 [hep-ph/0503132] [INSPIRE].

[45] R. Britto, B. Feng and P. Mastrolia, The Cut-constructible part of QCD amplitudes, Phys. Rev. D 73 (2006) 105004 [hep-ph/0602178] [INSPIRE].

[46] C. Anastasiou, R. Britto, B. Feng, Z. Kunszt and P. Mastrolia, D-dimensional unitarity cut method, Phys. Lett. B 645 (2007) 213 [hep-ph/0609191] [InSPIRE].

[47] P. Mastrolia, On Triple-cut of scattering amplitudes, Phys. Lett. B 644 (2007) 272 [hep-th/0611091] [INSPIRE].

[48] N.E.J. Bjerrum-Bohr, D.C. Dunbar and W.B. Perkins, Analytic structure of three-mass triangle coefficients, JHEP 04 (2008) 038 [arXiv:0709.2086] [INSPIRE].

[49] D. Forde, Direct extraction of one-loop integral coefficients, Phys. Rev. D 75 (2007) 125019 [arXiv: 0704.1835] [INSPIRE].

[50] S.D. Badger, Direct Extraction Of One Loop Rational Terms, JHEP 01 (2009) 049 [arXiv:0806.4600] [INSPIRE].

[51] P. Mastrolia, Double-Cut of Scattering Amplitudes and Stokes' Theorem, Phys. Lett. B 678 (2009) 246 [arXiv:0905.2909] [INSPIRE].

[52] E.W. Nigel Glover and C. Williams, One-Loop Gluonic Amplitudes from Single Unitarity Cuts, JHEP 12 (2008) 067 [arXiv:0810.2964] [INSPIRE].

[53] R. Britto and B. Feng, Solving for tadpole coefficients in one-loop amplitudes, Phys. Lett. B 681 (2009) 376 [arXiv:0904.2766] [INSPIRE].

[54] R. Britto and E. Mirabella, Single Cut Integration, JHEP 01 (2011) 135 [arXiv:1011.2344] [INSPIRE].

[55] D.A. Kosower and K.J. Larsen, Maximal Unitarity at Two Loops, Phys. Rev. D 85 (2012) 045017 [arXiv: 1108.1180] [INSPIRE].

[56] E.R. Speer, Generalized Feynman Amplitudes, Princeton University Press (1969).

[57] R.N. Lee and A.A. Pomeransky, Critical points and number of master integrals, JHEP 11 (2013) 165 [arXiv: 1308.6676] [INSPIRE].

[58] K. Matsumoto, Relative twisted homology and cohomology groups associated with Lauricella's $F_{D}$, arXiv: 1804.00366.

[59] M. Kreuzer and L. Robbiano, Computational Commutative Algebra 1, Computational Commutative Algebra, Springer Berlin Heidelberg (2008). 
[60] P. Aluffi and M. Marcolli, Feynman motives of banana graphs, Commun. Num. Theor. Phys. 3 (2009) 1 [arXiv:0807.1690] [INSPIRE].

[61] M. Marcolli, Motivic renormalization and singularities, Clay Math. Proc. 11 (2010) 409 [arXiv:0804 .4824] [INSPIRE].

[62] T. Bitoun, C. Bogner, R.P. Klausen and E. Panzer, Feynman integral relations from parametric annihilators, Lett. Math. Phys. 109 (2019) 497 [arXiv:1712.09215] [INSPIRE].

[63] K. Aomoto and M. Kita, Theory of Hypergeometric Functions, Springer Monographs in Mathematics, Springer Japan (2011).

[64] M. Kita and M. Yoshida, Intersection Theory for Twisted Cycles, Math. Nachr. 166 (1994) 287.

[65] S. Mizera, Status of Intersection Theory and Feynman Integrals, PoS MA2019 (2019) 016 [arXiv: 2002.10476] [INSPIRE].

[66] S.-J. Matsubara-Heo, Euler and laplace integral representations of gkz hypergeometric functions, arXiv:1904.00565.

[67] Y. Goto and S.-J. Matsubara-Heo, Homology and cohomology intersection numbers of gkz systems, arXiv:2006.07848.

[68] M. Argeri et al., Magnus and Dyson Series for Master Integrals, JHEP 03 (2014) 082 [arXiv: 1401.2979] [INSPIRE].

[69] K.J. Larsen and Y. Zhang, Integration-by-parts reductions from unitarity cuts and algebraic geometry, Phys. Rev. D 93 (2016) 041701 [arXiv:1511.01071] [INSPIRE].

[70] A. Primo and L. Tancredi, On the maximal cut of Feynman integrals and the solution of their differential equations, Nucl. Phys. B 916 (2017) 94 [arXiv:1610.08397] [InSPIRE].

[71] H. Frellesvig and C.G. Papadopoulos, Cuts of Feynman Integrals in Baikov representation, JHEP 04 (2017) 083 [arXiv:1701.07356] [INSPIRE].

[72] M. Harley, F. Moriello and R.M. Schabinger, Baikov-Lee Representations Of Cut Feynman Integrals, JHEP 06 (2017) 049 [arXiv:1705.03478] [INSPIRE].

[73] A.V. Smirnov, FIRE5: a C++implementation of Feynman Integral REduction, Comput. Phys. Commun. 189 (2015) 182 [arXiv:1408.2372] [InSPIRE].

[74] R.N. Lee, Presenting LiteRed: a tool for the Loop InTEgrals REDuction, arXiv:1212.2685 [INSPIRE].

[75] A. von Manteuffel and C. Studerus, Reduze 2 - Distributed Feynman Integral Reduction, arXiv: 1201.4330 [INSPIRE].

[76] P. Maierhöfer, J. Usovitsch and P. Uwer, Kira - A Feynman integral reduction program, Comput. Phys. Commun. 230 (2018) 99 [arXiv:1705.05610] [InSPIRE].

[77] D. Binosi, J. Collins, C. Kaufhold and L. Theussl, JaxoDraw: A Graphical user interface for drawing Feynman diagrams. Version 2.0 release notes, Comput. Phys. Commun. 180 (2009) 1709 [arXiv: 0811.4113] [INSPIRE].

[78] J.A.M. Vermaseren, Axodraw, Comput. Phys. Commun. 83 (1994) 45 [inSPIRE]. 